\title{
Controlling Pneumococcal Disease around the Globe
}

\author{
Invited Lecture Abstracts \\ ISPPD-0535 \\ Controlling Pneumococcal Disease Around the Globe
}

UPDATE FROM AFRICA

A. Von Gottberg ${ }^{1}$

${ }^{1}$ South Africa

South Africa introduced the seven-valent pneumococcal protein-polysaccharide conjugate vaccine (PCV-7) in 2009 using a novel 3-dose infant schedule $(6,14$ and 36 weeks). We assessed PCV-7 impact using national disease surveillance data, accounting for HIV prevention effects. We conducted active, national, laboratorybased surveillance for invasive pneumococcal disease (IPD) from 2005 through 2012, including serotyping and susceptibility testing. We calculated the percentage change in IPD incidence, focusing on individuals aged $<2$ and 25-44 years, between post-vaccine (2011 and 2012) and pre-vaccine years (average of 2005 through 2008). From 2005 through 2012, 35,192 IPD cases were identified. Rates of PCV-7 vaccine-serotype IPD among children $<2$ years declined by $89 \%$ in 2012 , from 32.1 cases per 100,000 person-years to $3.4 / 100,000$ (95\% confidence interval [Cl], $-92 \%$ to $-86 \%)$ while nonvaccine-serotype IPD increased non-significantly from 8.9/100,000 to 9.5/100,000 (+6\%; $95 \% \mathrm{Cl},-16 \%$ to $+23 \%)$. Among adults age 25 to 44 years, vaccine serotype IPD declined by $57 \%(95 \% \mathrm{Cl},-63 \%$ to-50\% [3.7/100,000 to 1.6/100,000]), compared with smaller nonvaccine serotype IPD declines $(3.9 / 100,000$ to $3.5 / 100,000 ;-11 \%, 95 \% \mathrm{Cl},-21 \%$ to $+4 \%)$. Among children $<2$ years, penicillin-nonsusceptible IPD rates decreased $(34.1 / 100,000$ to $6.1 / 100,000$ [-82\%; $95 \% \mathrm{Cl},-85 \%$ to-78\%]). The large reduction in disease caused by vaccine serotypes among both children and adults likely reflects substantial direct and indirect benefits from PCV-7 introduction. The smaller reduction in nonvaccine-serotype IPD suggests HIV prevention efforts are also playing a role in prevention of serious pneumococcal disease.

Conflict of interest

Oral Plenary Abstracts

ISPPD-0181

Controlling Pneumococcal Disease Around the Globe

\section{THE IMPACT OF PCV7/PCV13 SEQUENTIAL INTRODUCTION TO ISRAELI NIP ON NON-PNEUMOCOCCAL OTITIS MEDIA (OM) EPISODES NECESSITATING CULTURE}

\author{
R. Dagan ${ }^{1}$, S. Ben-Shimol ${ }^{1}$, E. Leibovitz ${ }^{1}$, N. Givon-Lavi ${ }^{1}$ \\ ${ }^{1}$ Pediatric Infectious Disease Unit, Soroka University Medical Center and Ben-Gurion University of the Negev, Beersheva, Israel
}

Background and Aims: Pre-licensure and post implementation PCV studies have demonstrated that PCV impact on recurrent, non-responsive and chronic OM cases (complex-OM; COMP-OM) was significantly larger (up to 40\%) than when all-cause OM or first OM were measured. COMP-OM is frequently caused by non-pneumococcal pathogens, often forming biofilm, in which non-typable Haemophilus influenzae (NTHi) is the most frequent. We assessed the impact of PCV7/PCV13 introduction on all-cause COMP-OM enriched OM episodes, as well as specifically pneumococcal, NTHi and culture-negative episodes.

Methods: These were previously described (Dagan et al, 49th IDSA, Abstr. 1344, 2012). The surveillance period was Jul-2004 through Jun-2013. PCV7 was introduced to the Universal Program (with catch-up) in Jul-2009 and has been gradually replaced by PCV13 since Nov-2010.

Results: Overall, 6,122 OM episodes were submitted for culture. $\geq 1$ factor associated with COMP-OM was present in $2,614 / 3,881$ (67.4\%). Incidence (per 1,000 children <2y) of PCV13-serotypes, all-pneumococcal, NTHi, and culturenegative episodes by 6 months interval is showed in Figure 1. The calculated reductions are shown in Table 1. Conclusion: All OM cases, including pneumococcal, NTHi and culture-negative episodes enriched with COMP-OM were markedly reduced in children $<2$ years after PCV7/PCV13 introduction. 


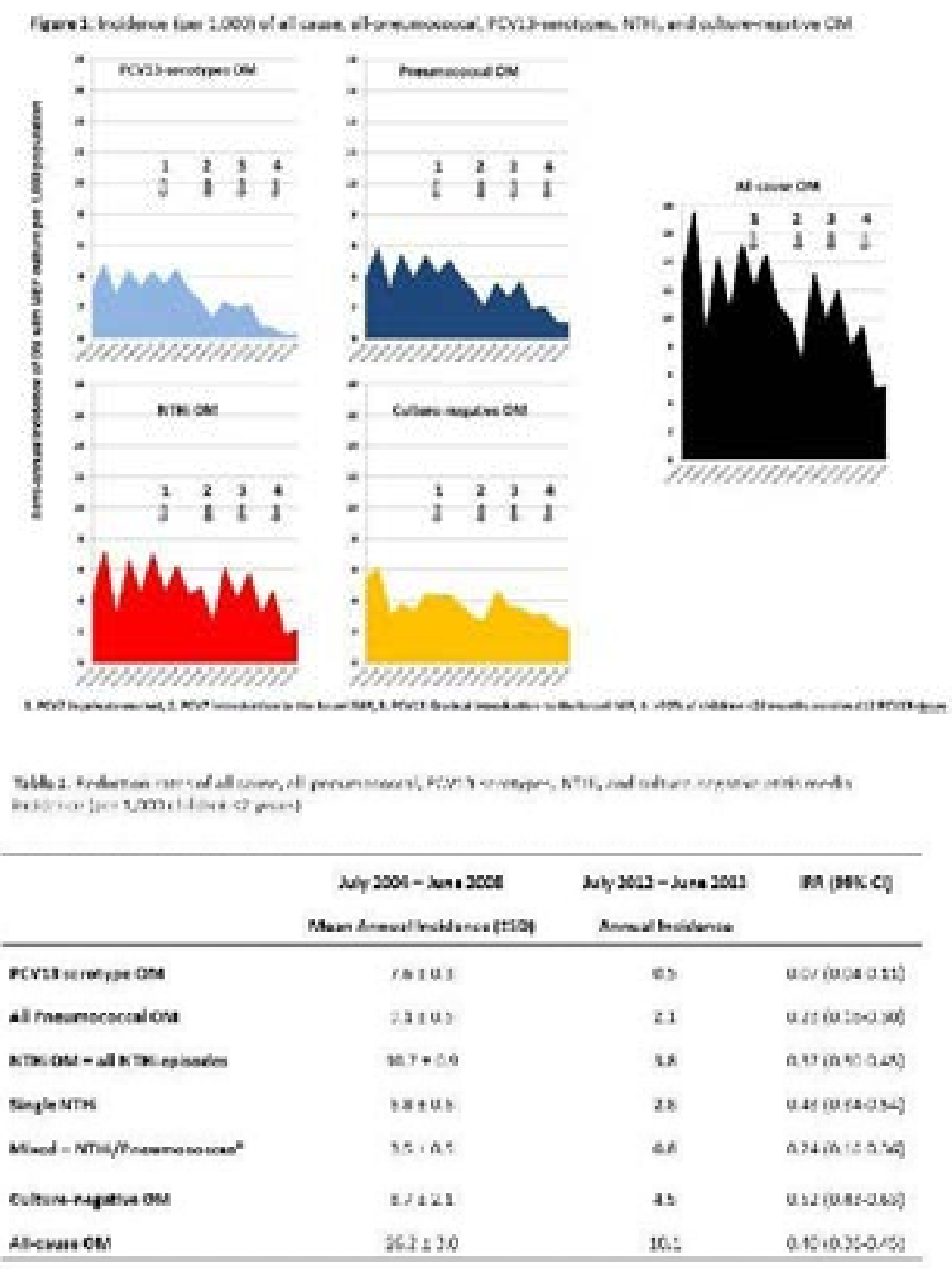

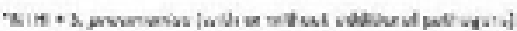

Conflict of interest

ISPPD-0560

Controlling Pneumococcal Disease Around the Globe

\section{EFFECTIVENESS OF 10-VALENT PNEUMOCOCCAL CONJUGATE VACCINE (PCV10) AGAINST INVASIVE PNEUMOCOCCAL DISEASE (IPD) DURING ONGOING NATIONAL VACCINATION PROGRAMME (NVP) IN FINLAND}

H. Rinta-Kokko ${ }^{1}$, K. Auranen ${ }^{1}$, A. Palmu², H. Nohynek ${ }^{1}$, P. Nuorti ${ }^{3}$, L. Siira ${ }^{4}$, M. Toropainen ${ }^{4}$, M. Virtanen ${ }^{4}$ J. Jokinen $^{1}$

${ }^{1}$ Department of Vaccination and Immune Protection, National Institute for Health and Welfare (THL), Helsinki, Finland; ${ }^{2}$ Department of Vaccination and Immune Protection, National Institute for Health and Welfare (THL), Tampere, Finland; ${ }^{3}$ Department of Infectious Disease Surveillance and Control, National Institute for Health and Welfare (THL), Tampere, Finland; ${ }^{4}$ Department of Infectious Disease Surveillance and Control, National Institute for Health and Welfare (THL), Helsinki, Finland

Background: PCV10 was introduced into the Finnish national vaccination programme in Sept 2010. Using the indirect cohort method on IPD cases accumulated until the end of 2013, we estimated vaccine effectiveness (VE) against IPD in vaccine-eligible children aged $>=3$ months.

Methods: Culture-confirmed, serotype-specific IPD cases were retrieved from the National Infectious Disease Register and vaccination histories and underlying comorbidities (riskfactors for IPD) from health records. Cases were grouped into PCV10-types (VT), PCV10-related types (serotypes of the same serogroups as PCV10-serotypes, VRT), and non-vaccine types (NVT). Cases were further categorized by their vaccination status (completely (>=2 doses)/ partly/unvaccinated). VE was estimated as 1 - odds of vaccination in cases with serotype/group category of interest (e.g. VT) versus odds of vaccination in NVT cases as controls.

Results: Table presents VE estimates for completely vaccinated children with and without stratification by comorbidity $(n=5 / 34)$. Among the partly vaccinated, the numbers of VT, VRT and NVT-cases were 5,0 and 2 , respectively. 


\begin{tabular}{|c|c|c|c|c|}
\hline & \multicolumn{2}{|c|}{ Number of cases } & \multicolumn{2}{|l|}{ VE (95\% CI) } \\
\hline & Vaccinated & Unvaccinated & Crude & Stratified \\
\hline VT & 1 & 8 & $98(72 \ldots 100)$ & 95 (47...99) \\
\hline 14 & 0 & 6 & $100(76 \ldots 100)$ & $100(76 \ldots 100)$ \\
\hline $19 \mathrm{~F}$ & 1 & 1 & $84(-1466 \ldots 100)$ & $70(-283 \ldots 98)$ \\
\hline VRT & 6 & 2 & $58(-598 \ldots 98)$ & $56(-232 \ldots 94)$ \\
\hline 19A & 5 & 1 & $32(-4611 \ldots 97)$ & $29(-631 . . .93)$ \\
\hline NVT & 15 & 2 & - & - \\
\hline All & 22 & 12 & - & - \\
\hline
\end{tabular}

Exact confidence intervals $(\mathrm{Cl})$ by Mantel-Haenszel method.

Conclusion: VE against VT-IPD is excellent three years after onset of PCV10 vaccination. The small number of cases precludes conclusive interpretation about VE against individual serotypes, except for serotype 14.

Conflict of interest

ISPPD-0562

Controlling Pneumococcal Disease Around the Globe

\title{
EFFECTIVENESS OF HIGHER VALENCY CONJUGATE VACCINES ON INVASIVE PNEUMOCOCCAL DISEASE IN EUROPE: PRELIMINARY RESULTS OF SPIDNET MULTICENTRE PROJECT
}

C. Savulescu ${ }^{1}$, G. Hanquet ${ }^{1}$, a. the SpIDnet group ${ }^{2}$

${ }^{1}$ Epidemiology Department, EpiConcept, Paris, France; ${ }^{2}$ Vaccine Preventable Disease Programme, European Centre for Disease Prevention and Control, Stockholm, Sweden

Background and Aims: The European Centre for Disease Control (ECDC) funded SpIDnet network commenced in 2012 to conduct active population-based surveillance for invasive pneumococcal disease (IPD) in 10 sites from 8 European countries. We used IPD surveillance data to measure the effectiveness of higher-valency pneumococcal conjugate vaccines (PCV10/13) against IPD in children.

Methods: We included children 2 months - 4 years with IPD reported to surveillance systems from January 2012 to August 2013 using a common protocol. We compared the proportion of vaccinated with at least one dose PCV10/13 among vaccine serotype IPD cases to that of IPD cases due to nonPCV13 serotypes. We computed effectiveness as (1-vaccination odds ratio)*100, adjusted for age, sex, underlying conditions and surveillance site (aVE). We reported aVE for PCV7, PCV13nonPCV7 and specific serotypes with 95\% confidence intervals (CI).

Results: We analysed data from 335 children, excluding records with missing serotype (135 records) and vaccination status (27 records). The aVE was $94.6 \%(95 \% \mathrm{Cl}: 79.9 ; 98.5)$ against PCV7 IPD $(n=123)$ and $87.1 \%(95 \% \mathrm{Cl}: 70.1 ; 94.4)$ against PCV13nonPCV7 IPD ( $n=188)$. The PCV13 aVE was $90.9 \%(95 \% \mathrm{Cl}: 67.4 ; 97.5)$ for serotype $19 \mathrm{~A}$ and $48.5 \%$ (95\% Cl: -99.8; 86.7) for serotype 3. The PCV10/13 aVE was $81 \%$ (95\%Cl: $28.6 ; 94.9)$ for serotype $1,95.2 \%(95 \% \mathrm{Cl}$ : $70.5 ; 99.2)$ for serotype 14 , and $97.8 \%(95 \% \mathrm{Cl}: 68 ; 99.8)$ for serotype $19 \mathrm{~F}$.

Conclusion: SpIDnet preliminary results suggest a good PCV10/13 effectiveness overall and by specific serotype (except serotype 3). Limitations may be related to possible heterogeneity due to medical practices or small sample size by site and deserve further investigation.

No conflict of interest

Oral Poster Abstracts

ISPPD-0487

Controlling Pneumococcal Disease Around the Globe

\section{UPDATED EFFECTIVENESS OF 13 VALENT PNEUMOCOCCAL CONJUGATE VACCINE IN ENGLAND AND WALES AND DERIVATION OF SEROTYPE SPECIFIC CORRELATES OF PROTECTION}

\author{
N.J. Andrews ${ }^{1}$, P. Kaye ${ }^{1}$, M.P.E. Slack ${ }^{1}$, L. Roalfe ${ }^{2}$, M. Zancolli², P. Burbidge ${ }^{2}$, E. Pearce ${ }^{2}$, E. Miller ${ }^{1}$, D. Goldblatt ${ }^{2}$ \\ ${ }^{1}$ Immunisation Hepatitis and Blood Safety, Public Health England, London, United Kingdom; ${ }^{2}$ Institute of Child Health, University College London, \\ London, United Kingdom
}

Background/Aims: The United Kingdom infant immunisation schedule incorporated PCV7 in 2006 and PCV13 in 2010. National surveillance has permitted the assessment of vaccine effectiveness. Nested immunogenicity studies in the population allowed us to derive correlates of protection (COP) for individual serotypes.

Methods: Vaccine effectiveness for PCV7 and PCV13 $(>/=2$ doses $<12 \mathrm{~m}$ or 1 dose $>12 \mathrm{~m})$ was assessed using the indirect cohort method. Immunogenicity data used to derive CoP was from local studies of PCV7 and PCV13. Sera 
were drawn at 5 months of age following 2 priming doses ( 2 and 4 months) and analysed for serotype specific lgG and a subset $(n=100)$ for OPA to 13 serotypes. Reverse cumulative distribution curves were derived for lgG and OPA titres and CoP calculated.

Results: Overall efficacy of PCV13 since introduction was $73.4 \%(95 \% \mathrm{Cl}, 56.8 \%-83.6 \%)$ with significant protection demonstrated for 4/6 additional serotypes. The aggregate VE for the 6 additional serotypes was $72.0 \%$ (53.8-83.0) and $86.9 \%$ (14.8-98.0) for the original PCV7 types. The aggregate CoP for all 13 serotypes was $0.99 \mu \mathrm{g} / \mathrm{ml}, 1.23 \mu \mathrm{g} /$ $\mathrm{ml}$ for the 6 additional, and $0.65 \mu \mathrm{g} / \mathrm{ml}$ for original PCV7 serotypes. Serotype specific IgG CoP ranged from $0.14 \mu \mathrm{g} /$ $\mathrm{ml}-1.03 \mu \mathrm{g} / \mathrm{ml}$ and functional correlates from 4-674 opsonic titre.

Conclusion: While an aggregate CoP of $0.35 \mu \mathrm{g} / \mathrm{ml}$ has been useful for licensing new PCVs, our data reveals the wide range of serotype-specific CoP. The relationship between IgG after priming and long term protection is likely to be more complex than initially envisaged and the success of $0.35 \mu \mathrm{g} / \mathrm{ml}$ as a threshold for licensing new vaccines needs to be better understood.

Conflict of interest

ISPPD-0465

Controlling Pneumococcal Disease Around the Globe

\section{IS REDUCTION IN PNEUMOCOCCAL MENINGITIS IN NON-VACCINATED AGE GROUPS IN MALAWI ATTRIBUTABLE TO THE INTRODUCTION OF 13-VALENT PNEUMOCOCCAL CONJUGATE VACCINE?}

N. Bar-Zeev ${ }^{1}$, D. Everett ${ }^{1}$, M. Alaerts ${ }^{1}$, A. Katsulukuta ${ }^{2}$, C. Mwansambo ${ }^{3}$, A. Costello ${ }^{4}$, J.R. Verani ${ }^{5}$, C.G. Whitney ${ }^{5}$, N.A. Cunliffe ${ }^{6}$, R.S. Heyderman ${ }^{7}$, N. French ${ }^{6}$

${ }^{1}$ Microbes Immunity \& Vaccines, Malawi-Liverpool-Wellcome Trust Clinical Research Programme, Blantyre, Malawi; ${ }^{2}$ EPI Programme, Ministry of Health, Lilongwe, Malawi; ${ }^{3}$ Principal Secretary, Ministry of Health, Lilongwe, Malawi; ${ }^{4}$ Institute for Global Health, University College London, London, United Kingdom; ${ }^{5}$ Respiratory Diseases Branch, Centers for Disease Control \& Prevention, Atlanta, USA; ${ }^{6}$ Institute for Infection \& Global Health, University of Liverpool, Liverpool, United Kingdom; ${ }^{7}$ Director, Malawi-Liverpool-Wellcome Trust Clinical Research Programme, Blantyre, Malawi

Background and Aims: The burden of pneumococcal disease in Malawi is high. The 13-valent conjugate vaccine (PCV13) was introduced in November 2011, with initial catch-up for infants <12 months. We utilized data from ongoing surveillance for pneumococcal meningitis to evaluate PCV13 impact in the 2 years post introduction.

Methods: We used time-series models to forecast future rates of all serotype pneumococcal meningitis based on 12 years of pre-PCV13 baseline data. After introduction we compared the observed against predicted case numbers. We further stratified our analysis for vaccine age-eligible children and adults.

Results: Before introduction we observed 645 (annual mean 54, SD 21) adult and 451 (mean 38, SD 14) infant cases, and respectively 60 (mean 30, SD 18) and 13 (mean 7, SD 5) cases since. Our models predicted a decline in pneumococcal meningitis even in absence of vaccine. Following vaccine introduction, overall case numbers were within our model predictions based on pre-PCV13 trends. In stratified analysis, case numbers post-introduction were lower than predicted for vaccine age-eligible infants; the numbers for adults were as predicted. We found no statistical difference in magnitude of forecast error by age-group.

Conclusions: Although pneumococcal meningitis incidence was decreasing before PCV13 introduction, our findings suggest vaccine direct effect contributing to further decline. In the 2 years following PCV13 introduction we found no evidence of indirect effect using non-serotyped age-disaggregated data. In the context of underlying secular trends, quantifying the overall indirect public health benefit attributable to infant vaccine will be challenging in the short term.

No conflict of interest

ISPPD-0059

Controlling Pneumococcal Disease Around the Globe

\section{EFFECTIVENESS OF 7 AND 13-VALENT PNEUMOCOCCAL CONJUGATE VACCINES FOR CONSOLIDATED PNEUMONIA IN HOSPITALIZED CHILDREN}

M. Hortal ${ }^{1}$, H. Laurani ${ }^{2}$, M. Meny ${ }^{3}$, M. Estevan ${ }^{4}$, F. Arrieta ${ }^{5}$

${ }^{1}$ Basic Sciences Program, PNUD/Nat. University, Montevideo, Uruguay; ${ }^{2}$ Advisor, Comisión Honoraria Antituberculosa (CHLA), Montevideo, Uruguay; ${ }^{3}$ Statistics, Intendencia Municipal, Montevideo, Uruguay; ${ }^{4}$ Radiology, Children's Hospital, Montevideo, Uruguay; ${ }^{5}$ Immunisation, Comisión Honoraria Antituberculosa (CHLA), Montevideo, Uruguay

Introduction: Pneumococcal conjugate vaccines (PCV7 and PCV 13) were introduced into National Immunization Program (NIP) of Uruguay since 2008 showing great impact against hospitalization due to consolidated pneumonia in children.

Objective: To estimate the effectiveness of each vaccine dose by establishing the changes in incidence of hospitalizations for consolidated pneumonia per person-year.

Methods: We followed 2008 cohort (vaccinated with PCV7) from January 1st, 2009, until March 2010 and cohort 2010 ( vaccinated with PCV13) until March 2013 The population based surveillance made available the clinical records of inpatients with consolidated pneumonia A unique NIP register provided data on vaccination status of every child from both cohorts Impact of PCVs was estimated by calculation of incidence density of hospitalization for consolidated pneumonia per 105 person-years.

Results: At December 2009, 97.7\% of 3,993 children in the 2008 cohort were vaccinated with at least one dose of PCV7 and there was a 55.7, 66.2 and 69.3\% reduction among vaccinated children after 1st, 2nd and 3rd dose 
respectively. December 2011, 99.8\% of 3,822 children in the 2010 cohort received at least one dose of PCV13. At the end of the observation period, there was a 69,79 and $85 \%$ reduction in hospitalization for consolidated pneumonia among vaccinated children after one of each dose.

Conclusion: This study demonstrates effectiveness of PCV7 and PCV13 administered in a 2+1 schedule in reducing hospitalizations for consolidated pneumonia in children $<24$ months of age in a selected area from Uruguay. Supported by an unrestricted Pfizer contract

Conflict of interest

ISPPD-0372

Controlling Pneumococcal Disease Around the Globe

\section{EVALUATING THE RELATIVE IMPACT OF INTRODUCING PCV13 WITH AND WITHOUT A CATCH-UP CAMPAIGN IN NHA TRANG, VIETNAM}

O. Le Polain de Waroux ${ }^{1}$, S. Flasche ${ }^{1}$, K. Takahashi ${ }^{2}$, K. Ariyoshi ${ }^{2}$, E.K. Mulholland ${ }^{1}$, D. Goldblatt ${ }^{3}$, Y.H. Choi ${ }^{4}$, D.D. Anh ${ }^{5}$, L.M. Yoshida ${ }^{2}$, W.J. Edmunds ${ }^{1}$

${ }^{1}$ Department of Infectious Disease Epidemiology, London School of Hygiene \& Tropical Medicine, London, United Kingdom; ${ }^{2}$ Institute of Tropical Medicine, Nagasaki University, Nagasaki, Japan; ${ }^{3}$ Institute of Child Health, University College London, London, United Kingdom; ${ }^{4}$ Modelling and Economics Unit Immunisation Hepatitis and Blood Safety Department, Public Health England, London, United Kingdom; ${ }^{5}$ Epidemiology, National Institute of Hygiene and Epidemiology, Hanoi, Vietnam

Background and Rationale: Although catch-up campaigns (CC) at the introduction of PCV into routine vaccination (RV) programmes may help more rapidly decrease disease burden, the additional vaccine doses required to conduct CCs not only add extra costs but also may delay the introduction of vaccines elsewhere, given limited vaccine supplies.

Methods: We explored the impact of CCs through a dynamic Susceptible-Infected-Susceptible model of carriage acquisition and clearance applied to the population of Nha Trang, Vietnam. Model fitting to pre-vaccination carriage data was done through a Markov Chain Monte-Carlo algorithm. Post-vaccination carriage was predicted for four potential CCs, namely RV only, and RV alongside a CC in children aged 12-23m (CC1), 4-11m (CC2) and 1-4 years (CC3). Estimates of invasive pneumococcal disease (IPD) were based on previous estimates of the disease propensity of vaccine type (VT) and non-vaccine type (NVT) serotypes.

Results: Catch-up campaigns lead to a more rapid decrease in VT carriage and IPD incidence across all age groups within the first 2 years, with similar reductions obtained with CC1 and CC2 and more rapid reductions with CC3. Compared to RV only, any delay in the implementation of CC1 and CC2 by more than 6 months, and of CC3 by over 12 months, would be inferior to a RV only strategy.

Conclusion: Catch-up campaigns reduce carriage and disease more rapidly. However, this has to be balanced against the implementation delay CC might introduce. Our model predictions suggest that such CC would only be beneficial with little or no introduction delay.

No conflict of interest

ISPPD-0304

Controlling Pneumococcal Disease Around the Globe

\section{A PNEUMOCOCCAL SEROTYPE 20 OUTBREAK AMONG THE HOMELESS IN ALBERTA, CANADA}

G. Tyrrell $^{1}$, C. Mangan², Q. Ibrahim³ ${ }^{3}$, J. Kakulphimp ${ }^{4}$, D. Hurteau ${ }^{4}$, M. Lovgren ${ }^{4}$, T. Marrie $^{5}$

${ }^{1}$ Laboratory Medicine and Pathology, UAlberta, Edmonton, Canada; ${ }^{2}$ Undergraduate Medical Education, UAlberta, Edmonton, Canada

${ }^{3}$ EPICORE Centre, UAlberta, Edmonton, Canada; ${ }^{4}$ Bacterial Typing Unit, Provinical Laboratory for Public Health, Edmonton, Canada; ${ }^{5}$ Faculty of Medicine, Dalhousie University, Halifax, Canada

Background: Laboratory and clinical surveillance of invasive pneumococcal disease in Alberta has been occurring since 2000. We describe a pneumococcal serotype 20 (PS20) outbreak among homeless cases primarily residing in northern Alberta recognized by this surveillance program.

Methods: Invasive pneumococcal disease (IPD) is a notifiable disease to Provincial Health Authorities. As such, the isolates from all cases of IPD are forwarded to the Provincial Laboratory for Public Health (Provlab) for serotyping and antimicrobial susceptibility testing (AST). In addition, restricted fragment length polymorphism (RFLP) analysis was done to look for relatedness. Once PS20 cases were identified, a clinical data collection form was used to gather clinical data from each case. This was compiled into a larger database of IPD and analyzed for common trends/ conditions.

Results: From January 1991 to December 2010 (228 months), there were 36 cases of IPD caused by PS20 in Alberta or 0.16 cases/month. From January 2011 to August 2013 ( 32 months), there were 63 cases of IPD caused by PS20 or 2 cases/month, a 12.5 fold increase. RFLP analysis by pulsed-field gel electrophoresis suggested the outbreak was clonal. Risk factors in comparison to nonPS20 cases of IPD included homelessness, alcoholism, illegal drug use and hepatitis $\mathrm{C}$ infection.

Conclusion: Alberta, Canada is experiencing an outbreak of IPD caused by a serotype 20 clone. The outbreak is centered primarily in the homeless population. Serotype 20 is not included in the PCV13 vaccine however, it is included in the PPV23 vaccine. 
ISPPD-0234

Controlling Pneumococcal Disease Around the Globe

\title{
INVASIVE PNEUMOCOCCAL DISEASE AMONG ADULTS AFTER ROUTINE INFANT CONJUGATE VACCINATION IN FINLAND - IMPLICATIONS FOR VACCINATION POLICY
}

\author{
J.P. Nuorti ${ }^{1}$, H. Rinta-Kokko ${ }^{2}$, M. Toropainen ${ }^{3}$, L. Siira ${ }^{3}$, M.J. Virtanen ${ }^{4}$, H. Nohynek ${ }^{2}$, A.A. Palmu², J. Jokinen ${ }^{2}$ \\ ${ }^{1}$ Department of Infectious Disease Surveillance and Control, National Institute for Health and Welfare (THL), Helsinki, Finland; ${ }^{2}$ Depart- \\ ment of Vaccines, National Institute for Health and Welfare (THL), Helsinki, Finland; ${ }^{3}$ Bacteriology Unit, National Institute for Health and Wel- \\ fare (THL), Helsinki, Finland; ${ }^{4}$ Epidemiologic Surveillance Unit, National Institute for Health and Welfare (THL), Helsinki, Finland
}

\begin{abstract}
Background and Aims: Ten-valent pneumococcal conjugate vaccine (PCV10) was introduced into Finnish national infant vaccination program in September 2010 using $2+1$ schedule. We evaluated changes in invasive pneumococcal disease (IPD) rates and serotype distribution among adults after routine infant vaccination with an estimated $>90 \%$ PCV10 uptake.

Methods: Culture-confirmed IPD cases $>18$ years $(n=2917)$ were identified through national, population-based laboratory surveillance. Denominator data were from the Population Information System. We compared age- and serotype-specific rates of IPD in 7/2012-6/2013 with those in 7/2006-6/2009 (before PCV10). Time period of a large cluster-randomized PCV10 trial was excluded.

Results: Overall annualized IPD rate decreased from 9.2 to 6.8/100,000 among persons 18-49 years; overall rates did not change significantly in age groups $50-64$ years $(19.5 / 100,000)$, and $>65$ years $(26.9 / 100,000)$. Incidence of PCV10 serotype IPD decreased significantly by $41.3 \%, 23.7 \%$ and $40.9 \%$ among persons $18-49,50-64$, and $>65$ years, respectively. Non-PCV10 types increased significantly by $40.0 \%$ and $28.3 \%$ among persons $50-64$ and $>65$ years. Rates of serotype 19A increased among persons $18-49$ and $>65$ years and serotype $22 \mathrm{~F}$ among those 50-64 years. During 7/2012-6/2013, serotypes covered in PCV10 and PCV13 caused $39.9 \%$ and $60.7 \%$ of IPD cases among persons $>50$ years, respectively; PPSV23 types accounted for $81.5 \%$ of cases.

Conclusion: The indirect PCV10 program impact on overall disease burden among adults was relatively small. This was due to shifting of pneumococcal serotype distribution from PCV10 to non-PCV10 types following routine infant vaccination. In the current epidemiologic situation older adults might benefit from direct PCV13 vaccination.
\end{abstract}

No conflict of interest

ISPPD-0258

Controlling Pneumococcal Disease Around the Globe

\section{EARLY IMPACT OF SEQUENTIAL PCV7/PCV13 INTRODUCTION TO NATIONAL IMMUNIZATION PEDIATRIC PLAN ON ADULTS INVASIVE DISEASE IN ISRAEL}

G. Regev-Yochay ${ }^{1}$, Y. Paran ${ }^{2}$, J. Bishara ${ }^{3}$, I. Oren ${ }^{4}$, M. Chowers ${ }^{5}$, Y. Ztiba ${ }^{6}$, V. Iston ${ }^{7}$, M. Weinberger ${ }^{8}$, D. Miron ${ }^{9}$, R. Da$\operatorname{gan}^{10}$

${ }^{1}$ Infectious Disease Unit, Sheba Medical Center, Ramat Gan, Israel; ${ }^{2}$ Infectious Disease Unit, Tel Aviv Medical Center, Tel Aviv, Israel; ${ }^{3}$ Infectious Disease Unit, Rabin Medical Center, Petach Tikva, Israel; ${ }^{4}$ Infectious Disease Unit, Rambam Medical Center, Haifa, Israel; ${ }^{5}$ Infectious Disease Unit, Meir Medical Center, Kfar Saba, Israel; ${ }^{6}$ Infectious Disease Unit, Barzilay Medical Center, Ashkelon, Israel; ${ }^{7}$ Infectious Disease Unit, Hillel Yaffe Medical Center, Hedera, Israel; ${ }^{8}$ Infectious Disease Unit, Assaf Harofe Medical Center, Rishon Lezion, Israel; ${ }^{9}$ Infectious Disease Unit, Rivka Ziv Medical Center, Zfat, Israel; ${ }^{10}$ Pediatric Infectious Disease Unit, Soroka University Medical Center, Beer Sheva, Israel

Background: PCV7 was introduced as universal childhood vaccination in Israel on Jul 2009 and was gradually replaced by PCV13 from Nov 2010. Here we report data on adult IPD, two years post PCV13 implementation. Methods: An ongoing nationwide active surveillance (all 27 laboratories performing blood cultures in Israel), providing all blood/CSF pneumococci from persons $>18 \mathrm{y}$ initiated on Jul2009. Capture-recapture method assured reporting of $>95 \%$ cases. All isolates were serotyped in one central laboratory. Medical history and outcomes were recorded in $\sim 90 \%$.

Results: Overall VT7/VT13 serotypes decreased by $69 \% / 55 \%$ within 4 years. This was accompanied by a $47 \%$ increase in nonVT13 strains. These changes were most apparent in winter season (Fig.1) and were seen in all age groups (Fig2), including in individuals with comorbidities. Non-VT serotypes that significantly increased were $12 \mathrm{~F}$, $15 \mathrm{~A}$ and $10 \mathrm{~A}$. A continuous reduction in isolates with penicillin $\mathrm{MIC}>0.06$ was observed $(26 \%$ to $11 \%, \mathrm{p}<0.001)$. 


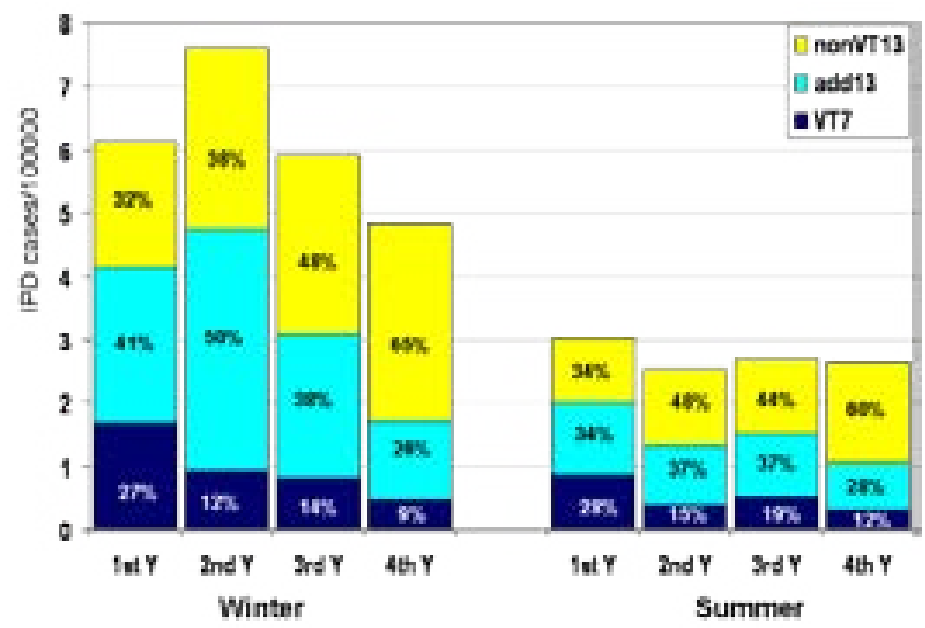

Figure 1: Winter vs. Summer changes in IPD incidence

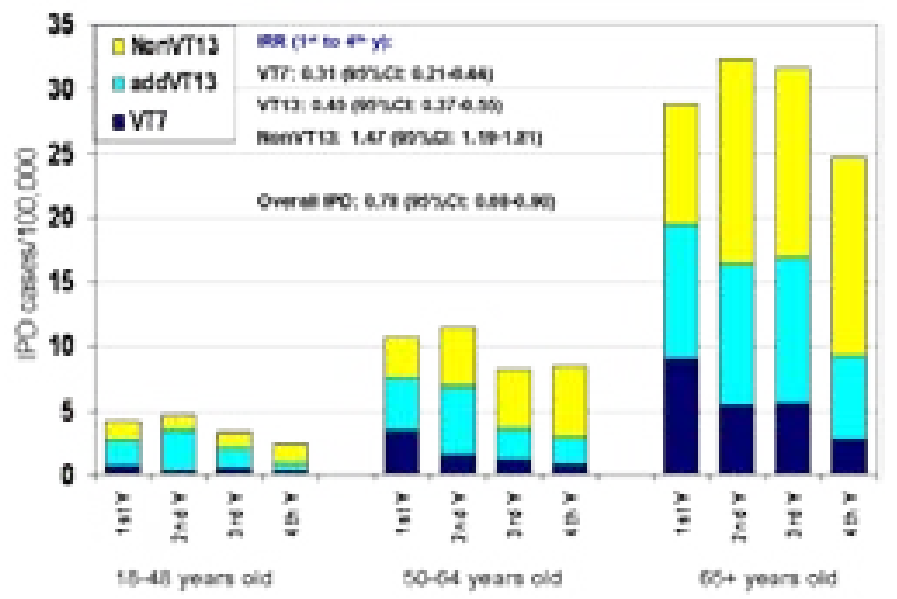

Figure 2: IPD Incidence rate by age, year and VT

Conclusion: Four years after PCV universal implementation in children, incidence of adult IPD caused by VT7 and VT13 decreased in all age groups. Despite increase in non-VT IPD, overall pneumococcal IPD decreased. However, additional follow-up is still needed to determine the long-term impact on adult IPD.

Conflict of interest

ISPPD-0288

Controlling Pneumococcal Disease Around the Globe

\section{EFFECTIVENESS OF TEN-VALENT PNEUMOCOCCAL CONJUGATE VACCINE AGAINST INVASIVE PNEUMOCOCCAL DISEASE IN BRAZIL: A MATCHED CASE-CONTROL STUDY}

C.M.A.S. Domingues ${ }^{1}$, J.R. Verani ${ }^{2}$, E.R. Montenegro ${ }^{1}$, M.C.C. Brandileone ${ }^{3}$, B. Flannery ${ }^{4}$, L.H. de Oliveira ${ }^{5}$, J.B. dos Santos ${ }^{6}$, J.C. Moraes ${ }^{7}$, Brazilian Pneumococcal Conjugate Vaccine Effectiveness Study Group ${ }^{1}$

${ }^{1}$ National Immunization Program, Brazilian Ministry of Health, Brasilia, Brazil; ${ }^{2}$ National Center for Immunization and Respiratory Diseases, Centers for Disease Control and Prevention, Atlanta, USA; ${ }^{3}$ National Reference Laboratory for Meningitis and Pneumococcal Infections, Adolfo Lutz Institute Sao Paulo State Health Secretariat, Sao Paulo, Brazil; ${ }^{4}$ Family Gender and Life Cycle, Pan American Health Organization, Brasilia, Brazil; ${ }^{5}$ Family and Community Health, Pan American Health Organization, Washington DC, USA; ${ }^{6}$ Center for Tropical Medicine, University of Brasilia, Brasilia, Brazil; ${ }^{7}$ Department of Social Medicine, School of Medical Sciences of Santa Casa, Sao Paulo, Brazil

Background: In 2010, Brazil introduced the 10-valent pneumococcal conjugate vaccine (PCV10). The schedule included primary doses at 2, 4 and 6 months plus a booster at 12 months. A single dose was offered for catch-up of unvaccinated children 12-23 months old. We conducted a case-control study to evaluate PCV10 effectiveness. Methods: Invasive pneumococcal disease (i.e., isolation of Streptococcus pneumoniae from blood, cerebrospinal fluid or other normally sterile site) was identified in children age-eligible for $\geq 1$ PCV10 dose through laboratory- and hospital-based surveillance in 10 Brazilian states. We aimed to identify 4 age- and neighborhood-matched controls for each case. We calculated effectiveness ([1- adjusted matched odds ratio] x 100\%) for vaccine-type and vaccinerelated serotypes (i.e., in the same serogroup as a vaccine serotype).

Results: Among 316 cases with available serotype and 1219 matched controls, the effectiveness of an ageappropriate PCV10 schedule was $83.8 \%$ (95\% confidence interval [Cl] 65.9-92.3) against vaccine serotypes and 
77.9\% (95\% Cl 4.0-91.7) against vaccine-related serotypes. Serotype-specific effectiveness was demonstrated for the two most common vaccine serotypes, 14 (VE: 87.7\%, 95\% $\mathrm{Cl} 60.8-96.0)$ and $6 \mathrm{~B}(82.8 \%, 95 \% \mathrm{Cl} 23.8-96.1)$, as well as serotype $19 \mathrm{~A}(82.2 \%, 95 \% \mathrm{Cl} 10.7-96.4)$, a serotype related to vaccine serotype $19 \mathrm{~F}$. A single catch-up dose among children aged $12-23$ months was effective against vaccine-type disease (68.0\%, $95 \% \mathrm{Cl} 17.6-87.6)$. No significant effectiveness was found against non-vaccine serotypes.

Conclusion: In the context of the routine immunization program in Brazil, PCV10 prevents invasive disease caused by vaccine serotypes and may provide cross-protection against some vaccine-related serotypes.

No conflict of interest

ISPPD-0045

Controlling Pneumococcal Disease Around the Globe

\section{INVASIVE PNEUMOCOCCAL DISEASE (IPD) IN ALASKA CHILDREN: IMPACT OF THE 13-VALENT PNEUMOCOCCAL CONJUGATE VACCINE (PCV13)}

M. Bruce ${ }^{1}$, R.S. Singleton ${ }^{2}$, L. Bulkow ${ }^{1}$, D. Bruden ${ }^{1}$, K. Rudolph ${ }^{1}$, T. Zulz ${ }^{1}$, M. Harker-Jones ${ }^{1}$, P. Gounder ${ }^{1}$, D. Hurlburt ${ }^{1}$ T. Hennessy ${ }^{1}$

${ }^{1}$ Arctic Investigations Program, Centers for Disease Control and Prevention, Anchorage, USA; ${ }^{2}$ Pediatrics, Alaska Native Medical Center, Anchorage, USA

Background: Historically, Alaska Native (AN) children have high rates of IPD. After statewide introduction of PCV7 in 2001, rates of IPD declined; however, non-PCV7 vaccine-type IPD, after an initial decrease, started increasing in 2004. In March 2010, PCV13 was introduced statewide (replacing PCV7) in Alaska. We evaluated the impact of PCV13 on IPD in children, 33 months after introduction.

Methods: Pneumococcal sterile site isolates, reported through state-wide surveillance, were serotyped using standard methods. Vaccine coverage was evaluated from electronic medical records at AN medical facilities. We defined a pre-vaccine time period 2005-2008 and post-vaccine time period April 2010-December 2012. Jan 2009March 2010 was excluded because PCV13 was introduced pre-licensure in one high-risk region in 2009.

Results: Among all Alaska children $<5$ years, PCV13 serotypes comprised $65 \%$ of IPD in the pre-vaccine period; $94.8 \%$ of AN children $19-35$ months of age had received 3 or more doses of PCV during the post-vaccine period. Among Alaska children $<5$ years, IPD rates decreased from 57.5 (pre) to 20.8 (post) per 100,000/yr $(p<0.001$ ); PCV13 serotype IPD decreased $81 \%$ from 35.6 to $6.5(p<0.001)$. Among AN children $<5$ years, IPD rates decreased from 156.6 to 57.3 ( $p<0.001$ ); PCV13 serotype IPD decreased 76\% from 91.4 to 21.5 ( $p<0.001)$; non-PCV13 serotype IPD did not decline significantly (54.1to 31.0, $p=0.09$ ).

Conclusion: 33 months after introduction of PCV13, PCV13-serotype IPD rates decreased in Alaska Native and nonNative children $<5$ years of age. There is no evidence of non-PCV13 serotype replacement IPD at this time. Further surveillance is needed.

No conflict of interest

ISPPD-0297

Controlling Pneumococcal Disease Around the Globe

\section{IMPACT ESTIMATION OF PNEUMOCOCCAL CONJUGATE VACCINES, ACCOUNTING FOR HETEROGENEITY AND REDUCING DATA NEEDS}

S. Flasche ${ }^{1}$, O. Le Polain de Waroux ${ }^{1}$, K.L. O’Brien², W.J. Edmunds ${ }^{1}$

${ }^{1}$ Department of Infectious Disease Epidemiology, London School of Hygiene \& Tropical Medicine, London, United Kingdom; ${ }^{2}$ Department of International Health International Vaccine Access Center, Johns Hopkins Bloomberg School of Public Health, Baltimore, USA

A priori estimates of pneumococcal conjugate vaccine (PCV) impact are complicated by serotype replacement. The impact of PCVs on invasive pneumococcal disease varies between settings; the sources of that variability are not entirely clear. Prediction models usually rely on disease incidence data, which in most of the developing world is unavailable and few have tested their predictive ability against surveillance data. Assuming PCV use results in a) vaccine serotype (VT) elimination in colonization, b) full serotype replacement in colonization and c) unchanged invasiveness of non vaccine serotypes (NVT) after vaccination, the incidence risk ratio (IRR) of IPD is: $\mathrm{IRR}=(\mathrm{Cvt} / \mathrm{Cnvt}+1) /(\mathrm{Dvt} / \mathrm{Dnvt}+1)$, where $\mathrm{C}$ and $\mathrm{D}$ are the proportion of VT and NVT carriage and disease respectively. The predictive ability of this method was tested by comparing predictions employing the serotype distributions in IPD1 and carriage ${ }^{2-15}$ to respective IPD impact surveillance estimates after 3 years of PCV use in children under 5 years of age ${ }^{1}$ using weighted linear regression. The predictive value for the observed impact of PCV7 on IPD in children was good (adjusted $R^{2}=0.97$ ) and the predictions accounted for the observed heterogeneity (see Figure). The relative disease impact of PCVs can be estimated a priori from the serotype distribution of a representative sample of carriers and IPD cases. The observed heterogeneity in disease impact likely arises mostly from differences in serotype distribution of disease and colonization before vaccination. 
No conflict of interest

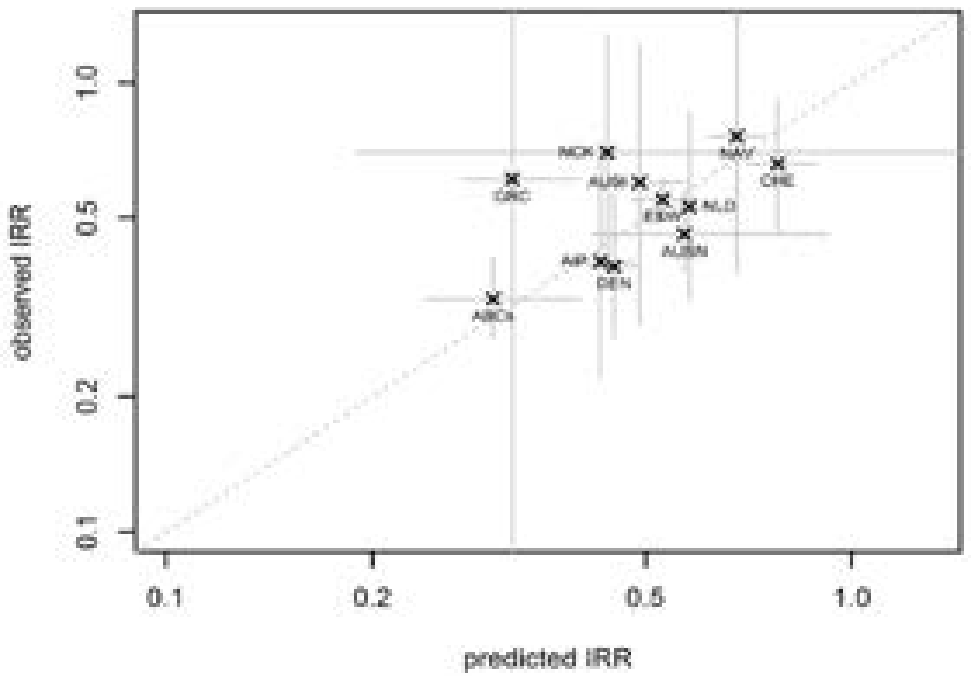

ISPPD-0292

Controlling Pneumococcal Disease Around the Globe

\section{EFFECTIVENESS OF PCV13 INTRODUCTION IN ARGENTINA IMMUNIZATION SCHEDULE: RESPIRATORY VIRUSES} INFLUENCE ON CONSOLIDATED PNEUMONIA (CP) 2001-2013. A PRELIMINARY TIME-SERIES ANALYSIS

A. Gentile ${ }^{1}$, M. Juarez ${ }^{1}$, M. Lucion ${ }^{1}$, M. Grobaporto ${ }^{1}$, V. Romanin ${ }^{1}$, N. Giglio ${ }^{1}$, J. Bakir ${ }^{1}$

${ }^{1}$ Epidemiology, R. Gutierrez Children's Hospital, Ciudad de Buenos Aires, Argentina

Background and Aims: PCV13 was introduced in Argentina's Immunization programme in 2012 for healthy children $<2$ years, with $2+1$ schedule. CP is a main outcome to measure PCVs effectiveness and respiratory viruses are an important cause of $C P$ in children. The aim of this study was to assess the effect of the vaccine introduction and the respiratory viruses influence on it.

Methods: We included all hospitalized WHO-defined pneumonia using prospective data from Acute Lower Respiratory Infection (ALRI) Surveillance Programme (2001-2013). Viral etiologic diagnosis was made by indirect immunofluorescence (VSR-Influenza (IF)-Parainfluenza-Adenovirus) and RT-PCR for IF. Time-series were performed calculating moving averages between prevaccination period (PreVP) 2001-2011 and postvaccination period (PostVP:2012-2013).

Results: We included 11240 children $<5$ y admitted with ALRI; 4964 had CP. Annual average of global CP admissions in PreVP:434.3 and PostVP:310.5(28.5\% reduction;95\%Cl:19.2-30.3\% $\mathrm{p}<0.001$ ). Annual average not viral-CP admissions PreVP:281.1 and post:178(36.7\% reduction;95\% Cl=29.3-43.3\% p<0.001). Annual average viralCP admissions PreVP:153.2 and post:133.5(12.8\% reduction;95\% $\mathrm{Cl}: 0.76-23.5 \mathrm{p}=0.035)$. Viral-CP etiology was proportionally higher after PCV13 introduction; in 2011 it represented 28\%of CP, 39.6\%in 2012 and $44.9 \%$ in 2013.
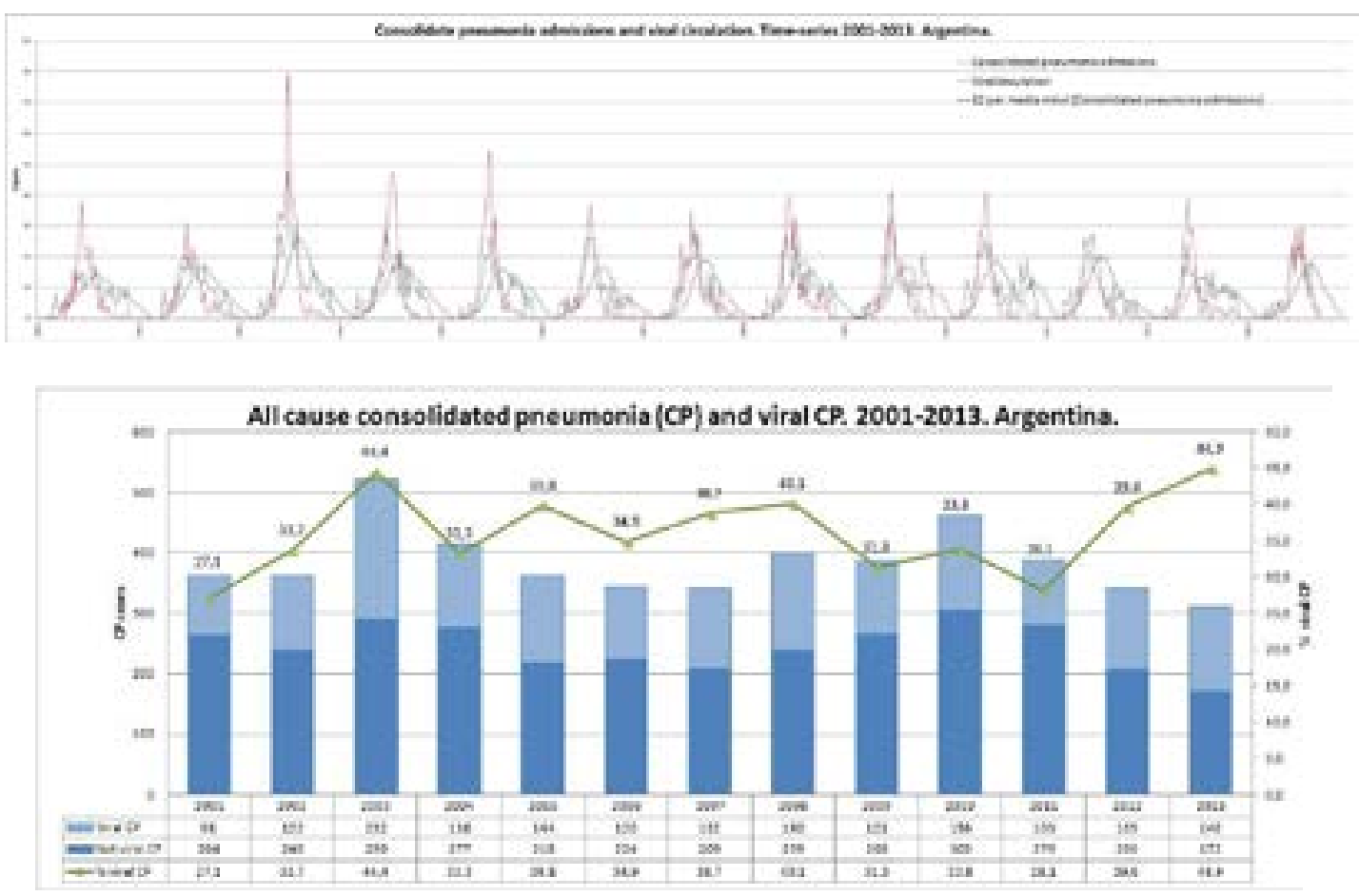
Conclusion: There was a significative global decline in hospitalized CP after PCV13 introduction, particularly in those without virus association. Epidemiological surveillance must continue to assess the impact of this vaccine and the viral influence on CP.

No conflict of interest

ISPPD-0413

Controlling Pneumococcal Disease Around the Globe

\title{
IMPACT OF THE 23VPPV BOOSTER IN INDIGENOUS CHILDREN IN NORTHERN AUSTRALIA
}

\author{
S. Jayasinghe ${ }^{1}$, C. Clayton ${ }^{1}$, R. Menzies ${ }^{1}$, V. Krause ${ }^{2}$, H. Cook $^{2}$, C. Giele ${ }^{3}$, D. Lehmann ${ }^{4}$, J. Hanna $^{5}$, P. Mclntyre ${ }^{1}$ \\ ${ }^{1}$ National Centre for Immunisation Research and Surveillance, The Children's Hospital at Westmead, Westmead, Australia; ${ }^{2}$ Centre for Dis- \\ ease Control, Department of Health Northern Territory Government, Darwin, Australia; ${ }^{3}$ Communicable Disease Control Directorate, Depart- \\ ment of Healht Western Australia, Perth, Australia; ${ }^{4}$ Telethon Institute for Child Health Research, The University of Western Australia, Perth, \\ Australia; ${ }^{5}$ School of Public Health Tropical Medicine \& Rehabilitation Sciences, James Cook University, Cairns, Australia
}

Introduction: From mid-2001, 7-valent pneumococcal conjugate vaccine (7vPCV) at 2, 4 and 6 months of age was fast-tracked for Indigenous children in Australia, given their disproportionately high rates of invasive pneumococcal disease (IPD). A 23-valent pneumococcal polysaccharide vaccine (23vPPV) booster was recommended in view of the diverse serotypes causing IPD in Indigenous children in northern Australia. From 2005, all non-indigenous children received 3 primary doses of 7vPCV without a booster.

Methods: We compared IPD notification rates by serotype category pre- and post-vaccine introduction among northern Australian Indigenous children and non-Indigenous children nationally aged $<5$ years. Vaccine coverage was obtained from the Australian Childhood Immunisation Register.

Results: Overall, IPD notification rates declined by $54 \%$ and $70 \%$ among Indigenous and non-Indigenous children respectively, from pre-vaccine introduction to 2010 , with $>90 \%$ reduction in 7VPCV-type IPD in both. IPD due to $23 \mathrm{v}-$ non-7v types increased by $144.5 \%(95 \% \mathrm{Cl} 102.9-196.3 \%)$ in non-Indigenous children (serotype 19A increased 264\% [ 182.8-373.7\%]) but only increased 1\% (19A decreased by 38\%) in Indigenous children. 23v-non-7v serotypes 33F, 22F and 12F increased in both. 23vPPV uptake by region ranged 57\%-79\%; 7vPCV uptake was $>85 \%$ in both groups but pre-2005, coverage increased more slowly in Indigenous children. About $70 \%$ of $23 \mathrm{v}-\mathrm{non}-7 \mathrm{v}$ IPD cases that were eligible for 23vPPV had received the vaccine.

Conclusion: Overall IPD decreased in Indigenous and non-Indigenous children. A smaller than expected increase in non-7v serotypes in Indigenous children was associated with 23vPPV booster. These findings may inform vaccination policy in countries with high IPD incidence and serotype diversity.

No conflict of interest

ISPPD-0322

Controlling Pneumococcal Disease Around the Globe

\section{3-VALENT PNEUMOCOCCAL CONJUGATE VACCINE (PCV13) BREAKTHROUGH INFECTIONS AND VACCINE FAILURES IN CHILDREN < 5 YEARS - UNITED STATES, 2010-2013}

L. Kim ${ }^{1}$, R. Gierke ${ }^{1}$, M. Farley ${ }^{2}$, W. Schaffner ${ }^{3}$, A. Thomas ${ }^{4}$, A. Reingold ${ }^{5}$, L. Harrison ${ }^{6}$, R. Lynfield ${ }^{7}$, C. Lexau ${ }^{7}$, S. Zansky $^{8}$, S. Petit ${ }^{9}$, M. Barnes ${ }^{10}$, M. Nichols ${ }^{11}$, L. McGee ${ }^{1}$, B. Beall ${ }^{1}$, C. Whitney ${ }^{1}$

${ }^{1}$ Respiratory Diseases Branch, Centers for Disease Control and Prevention, Atlanta, USA; ${ }^{2}$ Department of Medicine, Emory University and Atlanta VA Medical Center, Atlanta, USA; ${ }^{3}$ Department of Medicine, Vanderbilt University, Nashville, USA; ${ }^{4}$ Acute and Communicable Disease Prevention Program, Oregon Public Health Division, Portland, USA; ${ }^{5}$ Division of Epidemiology, University of California Berkeley, Berkeley, USA; ${ }^{6}$ Department of International Health, Johns Hopkins Bloomberg School of Public Health, Baltimore, USA; ${ }^{7}$ Infectious Diseases Division, Minnesota Department of Health, St. Paul, USA; ${ }^{8}$ Bureau of Communicable Disease Control, New York State Department of Health, Albany, USA; ${ }^{9}$ Epidemiology and Emerging Infections Program, Connecticut Department of Public Health, Hartford, USA; ${ }^{10}$ Emerging Infections Program, Colorado Department of Public Health and Environment, Denver, USA; ${ }^{11}$ Emerging Infections Program, New Mexico Department of Public Health, Santa Fe, USA

Background and Aims: The 13-valent pneumococcal conjugate vaccine (PCV13) was introduced in the U.S. in 2010. We sought to describe invasive pneumococcal disease (IPD) in children $<5$ years who developed disease despite receiving PCV13.

Methods: We used CDC's Emerging Infections Program/Active Bacterial Core surveillance to identify cases of IPD (sterile site isolate) occurring during April 2010-July 2013 in children $<5$ years who previously received $\geq 1$ PCV13 dose. Eligible cases had complete vaccination histories and serotype confirmed by Quellung. PCV13 breakthrough infections were defined as PCV13-type IPD in a child who received $\geq 1$ PCV13 dose. PCV13 failures (a subset of breakthrough infections) were defined as PCV13-type IPD in children who completed an age-appropriate, recommended vaccination schedule.

Results: Among 238 cases meeting the eligibility criteria, 41 (17\%) were breakthrough infections. Twenty (49\%) breakthrough infections occurred after receiving only 1 PCV13 dose, 7 after 2 doses, 8 after 3 doses, and 6 after 4 doses. Serotypes causing breakthrough infections were 19A $(n=30,73 \%), 3(n=9,22 \%), 7 F(n=1,2 \%)$, and $19 F(n=1$, $2 \%)$. Five vaccine failures (2\%) occurred, caused by serotypes $19 \mathrm{~A}(4)$ and $3(1)$. One had an underlying medical condition (asthma), and all required hospitalization and survived their illness.

Conclusions: Our findings suggest PCV13 is working as expected. While breakthrough infections occurred, they were most common after only 1 dose and tended to occur with serotype 19A, the most common serotype among children before PCV13 introduction. True vaccine failures have been uncommon. 
ISPPD-0259

Controlling Pneumococcal Disease Around the Globe

\section{ECONOMIC EVALUATION OF 13 VERSUS 10-VALENT PNEUMOCOCCAL CONJUGATE VACCINE IN THE NETHERLANDS}

A.W.M. Suijkerbuijk ${ }^{1}$, P. Vemer ${ }^{2}$, M.J. Knol ${ }^{1}$, A. Lugner ${ }^{1}$, M. Al ${ }^{2}$, A. Van der Ende ${ }^{3}$, E.A.M. Sanders ${ }^{4}$, H.E. De Melker ${ }^{1}$

${ }^{1}$ Center for Infectious Disease Control, National Institute for Public Health and the Environment, Bilthoven, Netherlands; ${ }^{2}$ Institute for Medical Technology Assessment, Erasmus University, Rotterdam, Netherlands; ${ }^{3}$ Medical Microbiology, Amsterdam Medical Center, Utrecht, Netherlands; ${ }^{4}$ Pediatric Immunology, University Medical Center Utrecht, Utrecht, Netherlands

Background and Aims: In the Netherlands, the 10-valent pneumococcal-conjugate vaccine (PCV10) is used in the National Immunisation Programme, in a 3+1 schedule. The aim of this study was to estimate the expected health outcomes, costs and incremental cost-effectiveness ratio (ICER) of routine vaccination with a 13-valent vaccine (PCV13) compared to the PCV10 vaccine, using the most recent available epidemiological data.

Methods: A Markov Model was used to calculate costs and effects of vaccinating one cohort of newborns. Expected effects include averted disease burden of meningitis, bacteremia, acute otitis media (AOM), and pneumonia. In the base case, vaccine price per dose was $€ 4.50$ lower and vaccine effectiveness regarding AOM was assumed to be higher for PCV10. Scenario and probabilistic sensitivity analyses were conducted to investigate the impact of indirect effects, number of doses, vaccine price and vaccine effectiveness. The analysis was performed from a societal perspective.

Results: The number of meningitis and bacteremia would be lowest using a PCV13 vaccination strategy. However, more cases of AOM would be averted in the PCV10 strategy, resulting in lower costs and more QALYs. Based on our assumptions, PCV10 would be the dominant strategy. Applying equal vaccine effectiveness for AOM, the ICER of implementing PCV13 compared with PCV10 would be $€ 38,000$ per QALY gained, and $€ 11,000$, including indirect effects.

Conclusion: The cost-effectiveness of pneumococcal vaccination highly depends on vaccine effectiveness against $A O M$, indirect effects of vaccination, and vaccine price. More long-term data is needed for more robust costeffectiveness estimates.

No conflict of interest

ISPPD-0467

Controlling Pneumococcal Disease Around the Globe

\section{IMPACT OF 13-VALENT PNEUMOCOCCAL CONJUGATE VACCINE (PCV13) AGAINST INVASIVE PNEUMOCOCCAL DISEASE (IPD) AMONG CHILDREN <5 YEARS OLD IN THE U.S.}

M. Moore ${ }^{1}$, T. Taylor ${ }^{1}$, T. Pondo ${ }^{1}$, M. Barnes ${ }^{2}$, S. Petit ${ }^{3}$, C. Holtzman ${ }^{4}$, R. Lynfield ${ }^{4}$, W. Schaffner ${ }^{5}$, L. Harrison ${ }^{6}$, S. Zansky $^{7}$, A. Reingold ${ }^{8}$, M.M. Farley ${ }^{9}$, K. Scherzinger ${ }^{10}$, A. Thomas ${ }^{11}$, E. Zell ${ }^{12}$, L. McGee ${ }^{1}$, B. Beall ${ }^{1}$, C. Whitney ${ }^{1}$

${ }^{1}$ Respiratory Diseases Branch, Centers for Disease Control \& Prevention, Atlanta, USA; ${ }^{2}$ Emerging Infections Program, Colorado Dept of Public Health and Environment, Denver, USA; ${ }^{3}$ Emerging Infections Program, Connecticut Department of Public Health, Hartford, USA; ${ }^{4}$ Emerging Infections Program, Minnesota Department of Health, St. Paul, USA; ${ }^{5}$ Department of Preventive Medicine, Vanderbilt Univeristy, Nashville, USA; ${ }^{6}$ Department of International Health, Johns Hopkins Univeristy, Baltimore, USA; ${ }^{7}$ Emerging Infections Program, New York State Department of Health, Albany, USA; ${ }^{8}$ Emerging Infections Program, University of California, Berkeley, USA; ${ }^{9}$ Atlanta Veterans Administration Medical Center, Emory University, Atlanta, USA; ${ }^{10}$ Emerging Infections Program, University of New Mexico, Albuquerque, USA; ${ }^{11}$ Emerging Infections Program, Oregon Public Health Division, Portland, USA; ${ }^{12}$ Stat-Epi Associates Inc., Atlanta, USA

Background and Aims: We evaluated the impact of PCV13 introduction in February 2010 on IPD among children $<5$ years.

Methods: IPD cases had sterile-site isolation of pneumococcus from $<5$ year-olds residing in any of 10 Active Bacterial Core surveillance sites during July 2004-June 2012. Isolates were serotyped at reference laboratories and classified as PCV7 (including 6A), PCV5 (19A, 7F, 3, 5, 1), and non-PCV13. We used pre-PCV13 observed cases in time-series models to forecast post-PCV13 incidence in the absence of PCV13 (counterfactual scenario). PCV13 impact was the difference between forecasted incidence and observed incidence. We derived confidence intervals using Monte-Carlo-simulations. Vaccine coverage was estimated from the National Immunization Survey.

Results: We identified 3,339 IPD cases among <5 year-olds; 2,898 (87\%) had serotyping information. Four-dose coverage of PCV (PCV7 and/or PCV13) was $82 \%$ by December 2012. Post-PCV13, overall and PCV5-type rates of IPD declined by $59 \%$ (95\% Cl 53-63\%) and $88 \%$ (95\% Cl 86-89\%), respectively. Cases of serotype 19A and 7F IPD declined by approximately $85 \%$ and $94 \%$, respectively. We were unable to detect statistically significant reductions in serotype 3 IPD. Over 4,000 cases of IPD were prevented nationally. During January-June 2012, PCV5 serotypes comprised $20 \%$ of all IPD in this age group with the most common serotypes being $19 \mathrm{~A}(14 \%), 22 \mathrm{~F}(12 \%), 33 \mathrm{~F}(11 \%)$, $35 \mathrm{~B}(8 \%), 10 \mathrm{~A}, 12 \mathrm{~F}$, and 15C (5\% each).

Conclusion: PCV13 appears highly effective at reducing IPD among children, especially in reducing incidence of disease caused by serotypes 19A and 7F.

No conflict of interest 
ISPPD-0424

Controlling Pneumococcal Disease Around the Globe

\title{
IDENTIFYING PROPORTIONATE AND DISPROPORTIONATE CHANGES IN NON-VACCINE SEROTYPE-SPECIFIC CARRIAGE AFTER PNEUMOCOCCAL CONJUGATE VACCINATION
}

\author{
M. Nurhonen ${ }^{1}$, K. Auranen ${ }^{1}$ \\ ${ }^{1}$ Department of Vaccination and Immune Protection, National Institute for Health and Welfare (THL), Helsinki, Finland
}

Serotype replacement in carriage after pneumococcal conjugate vaccination (PCV) is well established. Its implications on invasive pneumococcal disease (IPD) depend on the serotype-specific case-to-carrier ratios (probabilities of IPD per carriage episode) and the post-vaccination incidences of carriage of the non-vaccine types (NVT). The assumption of proportional increases in the carriage incidences of replacing NVTs has been used in predicting post-vaccination IPD. We propose a simple statistical method to validate the proportionality assumption and to identify potentially aberrant serotypes with respect to this assumption.

Published data on pre- and post-vaccination (PCV7) serotype distributions from three locations (UK, Massachusetts, Netherlands) were used. The serotype-specific NVT counts in each dataset were assumed to constitute multinomial samples with unknown serotype proportions. Pre- and post-vaccination serotype proportions were estimated with uncertainty bounds using a Bayesian approach. A regression line pertaining to the proportionality assumption with optimal fit to the observed post-pre pairs of serotype proportions was estimated, taking into account statistical uncertainty. Serotypes not conforming to the proportionality assumption were identified by probability scores describing the fit of the serotype-specific posterior distribution to the regression model.

In all three datasets, the proportional expansion model for carriage was solid as a general rule. Only a few serotypes did not satisfy the assumption of proportionality, most notably serotype 6A (vaccine-related) and 19A (disproportionately emerging in one data set). The consequences of future PCV vaccinations can be predicted using the proportionality assumption for the majority of serotypes while the carriage patterns of some serotypes may need special attention.

No conflict of interest

ISPPD-0461

Controlling Pneumococcal Disease Around the Globe

\begin{abstract}
IMPACT OF 10-VALENT PNEUMOCOCCAL CONJUGATE VACCINE (PCV10) AGAINST CLINICAL INVASIVE PNEUMOCOCCAL DISEASE (IPD) AMONG VACCINE-ELIGIBLE CHILDREN IN FINLAND
\end{abstract}

\author{
A.A. Palmu' ${ }^{1}$, T.M. Kilpi ${ }^{2}$, H. Rinta-Kokko², H. Nohynek², E. Ruokokoski², J.P. Nuorti ${ }^{3}$, J. Jokinen ${ }^{2}$ \\ ${ }^{1}$ Department of Vaccination and Immune Protection, National Institute for Health and Welfare, Tampere, Finland; Department of Vac- \\ cination and Immune Protection, National Institute for Health and Welfare, Helsinki, Finland; ${ }^{3}$ Department of Infectious Disease Surveil- \\ lance and Control, National Institute for Health and Welfare, Tampere, Finland
}

Background: PCV10 was introduced into the Finnish National Vaccination Programme (NVP) in September 2010 using a $2+1$ schedule $(3,5,12$ months). We evaluated the impact of PCV10 against clinical IPD among vaccineeligible children during the first two years after the NVP-introduction.

Methods: The target cohort eligible for NVP (children born from 06/2010-09/2012) was compared with two calendar-time and age-matched (3-30 months) cohorts before NVP introduction (Figure). Period 01/2009-08/2010 was excluded because of PCV10-trial conducted in Finland. Hospitals' in/outpatient discharge reports with ICD-10 diagnoses compatible with IPD (A40.3/B95.3/G00.1/M00.1) were collected from national Care Register and used for calculation of clinical IPD rates before and after NVP implementation. Episode duration of 90 days was used. The vaccination coverage is estimated to be around $95 \%$.

Results: The rate of all clinical IPD episodes was 138 in the combined control cohorts and 52/100,000 personyears in the target cohort. The relative rate reduction was $62 \%(95 \% \mathrm{Cl} 51$ to 68$)$ and the absolute rate reduction $86 / 100,000$ person-years in the target cohort compared with control cohorts combined.

Conclusion: This is the first report demonstrating PCV impact on clinical IPD during NVPs. Despite lower point estimate for relative rate reduction compared to that reported for culture-confirmed IPD (80\%), the absolute reduction was almost two-fold.

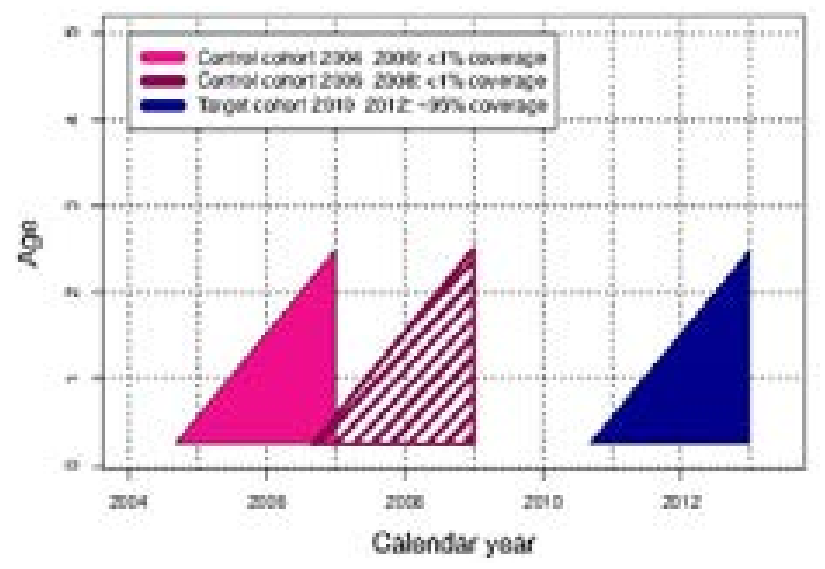

Figure: Cohorts for comparing PCV10- impact in the 


\section{Poster Abstracts}

ISPPD-0170

Controlling Pneumococcal Disease Around the Globe

\section{ANTIBODY PERSISTENCE AFTER PNEUMOCOCCAL CONJUGATE VACCINE (PCV13) IN 6-17 YEAR-OLD SICKLE CELL DISEASE (SCD) CHILDREN PREVIOUSLY VACCINATED WITH POLYSACCHARIDE (PPSV23)}

M. Abboud ${ }^{1}$, M. de Montalembert ${ }^{2}$, A. Fiquet ${ }^{3}$, N. Kaddah ${ }^{4}$, A. Piga ${ }^{5}$, D.C. Rees ${ }^{6}$, M.C. Driscoll7 , S. Al Hajjar ${ }^{8}$, Q. Jiang ${ }^{9}$, E.A. Emini ${ }^{9}$, W.C. Gruber ${ }^{9}$, A. Gurtman ${ }^{9}$, D.A. Scott ${ }^{9}$, B1851013 Study Group ${ }^{9}$

${ }^{1}$ Children's Cancer Centre, American University of Beirut, Beirut, Lebanon; ${ }^{2}$ Service de Pédiatrie, Hôpital Necker-Enfants Malades Service Pédiatrie, Paris, France; ${ }^{3}$ Vaccine Research, Pfizer Ltd., Maidenhead Berkshire, United Kingdom; ${ }^{4}$ Pediatric Hematology, Cairo University Hospital, Abu Alreesh Almoneera Cairo, Egypt; ${ }^{5}$ Ospedale San Luigi Gonzaga, OrbassanoTorino University, Torino, Italy; ${ }^{6}$ King's College Hospital, School of Medicine, London, United Kingdom; ${ }^{7}$ Division of Pediatric Hematology-Oncology, Children's Hospital at Montefiore, Bronx NY, USA; ${ }^{8}$ Department of Pediatrics, King Faisal Specialist Hospital and Research Centre, Riyadh, Saudi Arabia; ${ }^{9}$ Vaccine Research, Pfizer Inc, Pearl River NY, USA

Background and Aims: Children with sickle cell disease (SCD) have a significantly increased risk of invasive pneumococcal disease and mortality. Previously we reported immunogenicity data from a 2-dose regimen of PCV13 given 6-months apart to 6-17 year-old children with SCD who had previously received PPSV23. Herein, we present data collected 1 year after dose 2 .

Methods: Anti-pneumococcal polysaccharide immunoglobulin (IgG) geometric mean concentrations (GMCs) and opsonophagocytic activity (OPA) titers (GMTs) were determined for each blood draw. Newly diagnosed chronic medical conditions and serious adverse events (SAEs) were recorded.

Results: 158 subjects 13.3 ( \pm 3.08$)$ year-old were enrolled and vaccinated; 146 received dose $2 ; 87$ continued for 1 year. Antibody levels after doses 1 and 2 were generally comparable. Antibody levels declined over the year after dose 2; however, OPA GMTs for all serotypes (Table 1) and IgG GMCs (except serotype 3) remained above prevaccination levels.

Table 1: OPA GMTs (95\% Confidence Interval) - Evaluable Population

\begin{tabular}{|c|c|c|c|}
\hline Serotype & $\begin{array}{l}\text { Pre-vaccination } \\
n=95-131\end{array}$ & $\begin{array}{l}\text { 1-month post-dose } 2 \\
n=89-118\end{array}$ & $\begin{array}{l}\text { 1-year post-dose } 2 \\
n=55-73\end{array}$ \\
\hline 1 & $7 \quad(5.7-8.8)$ & $78 \quad(59.5-101.2)$ & $25 \quad(17.5-35.4)$ \\
\hline 3 & $13 \quad(10.1-17.5)$ & $105 \quad(87.2-127.2)$ & $31 \quad(22.6-42.6)$ \\
\hline 4 & 215 (129.6-357.2) & 3051 (2536.7-3670.3) & 1107 (764.4-1602.2) \\
\hline 5 & 10 (7.8-13.9) & $273 \quad(213.9-349.2)$ & $102 \quad(70.5-148.0)$ \\
\hline $6 \mathrm{~A}$ & 246 (149.0-404.8) & 7633 (6439.6-9048.6) & 2485 (1921.2-3214.3) \\
\hline $6 B$ & 626 (377.5-1037.4) & $7601 \quad(6392.6-9038.6)$ & $3412 \quad(2746.4-4238.7)$ \\
\hline $7 F$ & 344 (220.5-537.9) & $3723 \quad(3276.2-4230.1)$ & 2166 (1861.4-2519.3) \\
\hline $9 \mathrm{~V}$ & 234 (137.6-398.7) & $3467 \quad(2784.0-4317.6)$ & $1690(1157.8-2465.5)$ \\
\hline 14 & $628(425.8-925.7)$ & 2081 (1770.5-2446.0) & 1595 (1281.6-1984.9) \\
\hline $18 \mathrm{C}$ & 426 (235.7-771.4) & $5271 \quad(4267.8-6510.1)$ & 1604 (1107.2-2324.2) \\
\hline $19 A$ & 137 (100.0-187.4) & 1314 (1084.4-1592.6) & $(449.4-771.1)$ \\
\hline $19 F$ & $94 \quad(55.0-160.7)$ & 1507 (1139.9-1992.2) & $620 \quad(376.6-1020.4)$ \\
\hline $23 \mathrm{~F}$ & 34 (21.5-54.8) & $2330 \quad(1880.4-2887.0)$ & $(654.2-1306.1)$ \\
\hline
\end{tabular}

Most SAEs were due to vaso-occlusive crises. No deaths occurred.

Conclusion: Children with SCD previously vaccinated with PPSV23 responded well to PCV13. At 1 year after dose 2, antibodies persisted above PCV13 pre-vaccination levels for all serotypes.

Conflict of interest 
ISPPD-0032

Controlling Pneumococcal Disease Around the Globe

\title{
ACTIVITY OF BARK EXTRACTS OF TWO MEDICINAL PLANTS ON STREPTOCOCCUS PNEUMONIAE AND KLEBSIELLA PNEUMONIAE
}

\author{
K.O. Akinyemi ${ }^{1}$, O.K. Oluwa ${ }^{1}$, A.O. Coker $^{2}$ \\ ${ }^{1}$ Microbiology, Lagos State University Ojo Lagos, Lagos, Nigeria; ${ }^{2}$ Medical Microbiology and Parasitology, College of Medicine, University of La- \\ gos, Lagos, Nigeria
}

Background and Aims: The increasing incidence of death associated with respiratory tract infection in developing countries continues to draw the attention of the health care provider. Conventional antibiotics are no longer effective against some respiratory tract pathogens and the newer drugs are highly expensive and unaffordable for the teaming population in developing countries, thus resulting in the a high patronage of herbal medicine. This study was initiated to determine anti-pneumococcal activity of Bridelia ferruginea, and Garcina kola.

Methods: The aqueous and ethanolic bark extracts of the two medicinal plants Bridelia ferruginea, and Garcina kola were screened for anti-pneumococcal activity (Streptococcus pneumoniae, Klebsiella pneumoniae) using standard procedures.

Results: The results revealed that only the ethanolic extracts of Garcina kola inhibited the strains of these pathogens tested. The Minimum Inhibitory Concentration (MIC) recorded for the pathogens ranged from $18 \mathrm{mg} / \mathrm{ml}$ to $24 \mathrm{mg} / \mathrm{ml}$. Garcinia kola showed positive reactions to alkaloids, tannins, flavonoids and anthraquinones but in variable degrees.

Conclusion: This study showed that Garcinia kola may serve as potential natural source of drugs for respiratory diseases, thus justifying the efficacies claimed by traditional medical practitioners.

No conflict of interest

ISPPD-0247

Controlling Pneumococcal Disease Around the Globe

\section{PNEUMONIA AND MENINGITIS HOSPITALIZATIONS BEFORE AND AFTER PCV IMPLEMENTATION IN THE NATIONAL VACCINATION SCHEDULE IN URUGUAY}

M.C. Pirez ${ }^{1}$, G. Giachetto ${ }^{1}$, G. Algorta ${ }^{2}$, A. Cedres $^{1}$, H. Sobrero ${ }^{1}$, M. Sanchez Varela ${ }^{3}$, A. Varela ${ }^{4}$, J. Galazka ${ }^{5}$, T. Picón ${ }^{5}$, M. Uría ${ }^{5}$, C. Arocena ${ }^{5}$, S. Gutierrez ${ }^{6}$, A.I. Boix 6 , G. Sotelo ${ }^{6}$, M.C. Ferreiro ${ }^{7}$, M. Castro ${ }^{7}$, A. Montano ${ }^{1}$

${ }^{1}$ Clinicas Pediatricas, Facultad de Medicina. Universidad de la República, Montevideo, Uruguay; ${ }^{2}$ Bacteriología y Virología, Facultad de Medicina. Universidad de la República, Montevideo, Uruguay; ${ }^{3}$ Pediatria, British Hospital, Montevideo, Uruguay; ${ }^{4}$ Laboratorio, CHPR ASSE, Montevideo, Uruguay; ${ }^{5}$ Pediatria, Asociación Española ASESP, Montevideo, Uruguay; ${ }^{6}$ Pediatria, Centro Asistencia SMU, Montevideo, Uruguay

${ }^{7}$ DIVISA, Ministerio de Salud Publica, Montevideo, Uruguay

Background: Streptococcus pneumoniae was the most common cause of bacterial community-acquired pneumonia (CAP) and meningitis. In 2008 and 2010 PCV7 and PCV13 were incorporated ( $2+1$ schedule) for $<2$ years. Catch up were offered. High coverage (96\%) with PCV13 3 doses was achieved.

Aim: To compare hospitalization rates for CAP and pneumococcal meningitis (PM) three years before and three after universal vaccination with PCV7/PCV13 in 4 health care centers in Montevideo.

Methods: Design: retrospective study. Patients: children 0 month-14 years hospitalized at one public health provider (HP-CHPR) and three non public (ASESP, British Hospital, CASMU). Outcomes: Annual rates/10,000 discharges $(95 \% \mathrm{Cl})$ for CAP and PM before PCV7 vaccination (2005-2007), the year of vaccine implementation (2008), and after (2009-2011). Doses of PCV7/PCV13 and serotypes were described. Data were obtained from hospitals databases and vaccination records.

Results: 123,283 children were hospitalized for all causes $(91,227$ in HP-CHPR). Rates (95\%Cl) for CAP prevaccination and after vaccination were: HP-CHPR: 885 (859-912) and 314 (295-333), 65\% reduction. Other providers: 564 (525-604) and 323 (294-353), 43\% reduction. In the whole period 409 children with Pneumococcal-CAP (P-CAP) were hospitalized in HP-CHPR and 56 in the others. After PCV introduction 111 P-CAP were hospitalized. 102/111 strains were serotyped, 12 PCV7 serotypes, 66 additional and 26 non vaccine serotypes. Vaccine failure was observed in 4 children (serotypes 3, 23F, 1, 19A).

After PCV introduction, only one child completely vaccinated (with spinal fluid fistula) developed 2 meningitis episodes (6B, 19A).

Conclusion: CAP and PM rates decrease after PCV vaccination in different children populations.

Conflict of interest 
ISPPD-0439

Controlling Pneumococcal Disease Around the Globe

\section{THE BURDEN OF INVASIVE PNEUMOCOCCAL DISEASE AMONG OLDER PATIENTS IN THE ERA OF CONJUGATE VACCINE}

J. Azevedo ${ }^{1}$, M. Soares Santos ${ }^{1}$, V. Santos Galvão' ${ }^{1}$, E. Cunegundes Escobar ${ }^{1}$, R. Vilasboas ${ }^{2}$, S. Machado Cordeiro ${ }^{3}$, M. Galvão Reis ${ }^{1}$, A. Icksang Ko ${ }^{4}$, L. Carvalho Campos ${ }^{1}$, J. Neves Reis ${ }^{1}$

${ }^{1}$ Centro de Pesquisas Gonçalo Moniz, Fundação Oswaldo Cruz, Salvador, Brazil; ${ }^{2}$ Laboratório Central de Análises Clínicas, Secretaria de Saúde do Estado da Bahia, Salvador, Brazil; ${ }^{3}$ Faculdade de Farmácia, Universidade Federal da Bahia, Salvador, Brazil; ${ }^{4}$ Division Head Epidemiology of Microbial Disease, Yale School of Public Health, New Haven, USA

Background and Aims: A conjugate vaccine targeting 10 pneumococcal serotypes was licensed for young children in 2010 in Brazil. The aim of this study was to determine among adults aged 50 years or older whether incidence of invasive pneumococcal disease (IPD) have changed over the 3 years since pneumococcal conjugate vaccine licensure.

Methods: Prospective laboratory surveillance for IPD was performed from 2008 to 2013 in Salvador, Brazil. Incidence was compared for the 2 and 3 year period preceding and following pneumococcal vaccination. Capsular serotype was determined by multiplex PCR and/or Quellung reaction.

Results: Incidence of invasive pneumococcal disease among adults aged 50 years or older declined $20 \%$ from 0.36 cases/100.000 in 2008-2012 to 0.29 in 2010-2013. Among those aged 60 years or older, the incidence decreased $23 \%$ from 0.26 to 0.20 per $100,000 /$ inhabitants in pre- and post-PCV10 period. The most frequent pneumococcal vaccine related serotypes in the post-PCV10 were: 6A/B (17\%), $14(5.9 \%)$ and $19 \mathrm{~F}(5.9 \%)$. The non-PCV10 serotypes causing IPD in the period following vaccine implementation were: 7C (17\%), $3(13 \%), 19 \mathrm{~A}(8.7 \%)$ and $12 \mathrm{~F}(8.7 \%)$. Conclusion: Our findings indicate that use of conjugate vaccine in children may be reducing the rate of disease in older adults. Policy makers would consider the benefits seen in older adults to recommend pneumococcal conjugate vaccine in this age group.

Acknowledgments: Conselho Nacional de Desenvolvimento Científico e Tecnológico (\#482755/2010-5), Programa Estratégico de Apoio à Pesquisa em Saúde (PAPES\#407551/2012-3) and Fundação Oswaldo Cruz.

No conflict of interest

ISPPD-0354

Controlling Pneumococcal Disease Around the Globe

\section{7-VALENT PNEUMOCOCCAL CONJUGATE VACCINE: IMPACT ON EPIDEMIOLOGY OF PNEUMOCOCCAL MENINGITIS IN AUSTRALIA}

S. Bag ${ }^{1}$, S. Jayasinghe ${ }^{1}$, H. Wang ${ }^{1}$, H. Gidding ${ }^{2}$, F. Beard ${ }^{1}$, P. Mclntyre ${ }^{3}$

${ }^{1}$ Immunisation research, National Centre for Immunisation Research and Surveillance, Westmead, Australia; ${ }^{2}$ Immunisation research, National Centre for Immunisation Research and Surveillance and University of New South Wales, Sydney, Australia; ${ }^{3}$ Immunisation research, National Centre for Immunisation Research and Surveillance and University of Sydney, Sydney, Australia

Background: Following the introduction of a 7-valent pneumococcal conjugate vaccine (7vPCV) program in Australia for all infants in 2005, the incidence of invasive pneumococcal disease (IPD) decreased significantly. 7vPCV was replaced with 13-valent pneumococcal conjugate vaccine (13vPCV) in July 2011.

The most severe form of IPD is pneumococcal meningitis, which has a high case-fatality rate. Significant decreases in pneumococcal meningitis caused by 7VPCV serotypes have been reported in the United States and the United Kingdom, both in children and older age groups, following introduction of universal infant 7vPCV programs. Aims: To assess the impact of 7vPCV on pneumococcal meningitis epidemiology in Australia.

Methods: We examined trends in pneumococcal meningitis from 2004 to 2010 using Australian notification data. Analysis was stratified by age and serotype.

Results: In the $<5$ year age group, there was a $96 \%$ decrease in the number of meningitis cases caused by $7 \mathrm{vPCV}$ serotypes between the periods before (2004; 36 cases) and after7vPCV introduction (2007-2010; annual average 1.3 cases) ( $p<0.0001$ ). A non-significant $71 \%$ decrease ( 7 to 2 cases; $p=0.06$ ) was observed in the $\geq 65$ year old group over this period. There was a moderate increase in meningitis caused by non-7vPCV serotypes in both age groups ( $<5$ year olds: 5 to $16, p=0.0001 ; \geq 65$ year olds: 1.3 to $7.8, p=0.0001$ ), predominantly due the extra serotypes found in $13 \mathrm{vPCV}$.

Conclusion: Our study documents a significant decrease in pneumococcal meningitis in young children in Australia following introduction of the 7vPCV program. Ongoing surveillance is important to assess the impact of 13vPCV introduction.

No conflict of interest 
ISPPD-0289

Controlling Pneumococcal Disease Around the Globe

\title{
SURVEILLANCE OF INVASIVE PNEUMOCOCCAL DISEASES IN VENETO REGION, ITALY
}

\author{
T. Baldovin ${ }^{1}$, F. Russo ${ }^{2}$, R. Lazzari ${ }^{1}$, C. Bertoncello ${ }^{1}$, P. Furlan ${ }^{1}$, S. Cocchio ${ }^{1}$, V. Baldo ${ }^{1}$ \\ ${ }^{1}$ University of Padova Dept. of Molecular Medicine, Section of Public Health Laboratory of Hygiene and Public Health, Padova, Italy; ${ }^{2}$ Veneto Re- \\ gion Direction of Prevention, Promotion and Development of Hygiene and Public Health Service, Venice, Italy
}

Background and Aims: Streptococcus pneumoniae is characterized by $>90$ serotypes and it is a major cause of morbidity and mortality worldwide, especially in children $<5$ years old and the elderly. It occurs mostly as meningitis, pneumonia and bacteremia. This study aims to describe the epidemiology of invasive pneumococcal disease (IPD) in the Veneto Region (Northeast Italy).

Methods: Data were collected through a system of active surveillance of invasive bacterial diseases based on microbiology laboratories of the hospitals and local health authorities.

Results: In January 2007-June 2013 period, the Surveillance System of the Veneto Region received 963 IPD notifications. The notification rate was $3.0 / 100,000$. The age distribution show a higher incidence in subjects $<5$ $y$ old $(7.4 / 100,000)$ and in $\geq 65$ y old age group $(7.2 / 100,000)$. A decreased IPD incidence in children $<5$ y old is pointed out from the last quarter of 2009 , declining from 5.4 to $1.7 / 100,000$ in the first quarter of 2013 ( $p<0.001$ ); for the $\geq 65$ y old age group, the notification rate trend shows a slow increase in the analyzed period ( $p=n$.s.). A total number of 499 isolates were typified: $69.3 \%$ belongs to PCV13 serotypes, $17.6 \%$ to the additional PPSV 23 serotypes; $13.0 \%$ of remaining isolates belongs to strains not included in the available vaccines. In the analyzed period, 68 deaths occurred, with a lethality rate of $7.1 \%$; the $67.6 \%$ of deaths occurred in subjects $\geq 65$ y old

Conclusion: Current strategies have significantly reduced the IPD burden in subjects $<5$ y old Instead in the elderly, it is necessary a change of the vaccination strategy.

No conflict of interest

\section{DECLINE IN INCIDENCE AND MORTALITY OF INVASIVE PNEUMOCOCCAL DISEASE AFTER INTRODUCTION OF 13-VALENT CONJUGATE CHILDHOOD VACCINATION}

Z. Barrella Harboe ${ }^{1}$, T. Dalby ${ }^{1}$, D. Weinberger ${ }^{2}$, T. Benfield ${ }^{3}$, K. Mølbak ${ }^{4}$, H.C. Slotved ${ }^{5}$, C. Suppli ${ }^{4}$, H.B. Konradsen ${ }^{5}$, P. Valentiner-Branth ${ }^{4}$

${ }^{1}$ Meningitis and Respiratory Tract Infections, Statens Serum Instítut, Copenhagen, Denmark; ${ }^{2}$ Yale School of Medicine, Department of Epidemiology of Microbial Diseases Yale School of Public Health, New Haven Connecticut, USA; ${ }^{3}$ Department of Infectious Diseases, Hvidovre Hospital and Clinical Research Center, Copenhagen, Denmark; ${ }^{4}$ Department of Infectious Disease Epidemiology, Statens Serum Institut, Copenhagen, Denmark; ${ }^{5}$ Meningitis and Respiratory Tract Infections, Statens Serum Institut, Copenhagen, Denmark

Background: We wanted to explore PCV13's effect in reducing IPD-attributable morbidity and mortality, and whether serotype-specific changes were attributable to PCV13's introduction.

Methods: Population-based cohort study based on the linkage of national laboratory surveillance data on IPD and several national registries. We measured changes in IPD-incidence and mortality during baseline (2000-2007), PCV7 (2008-2010) and PCV13 (2011-2012) periods.

Results: We observed a $17 \%(95 \% \mathrm{Cl}, 13 \%-22 \%)$ reduction in the total incidence of IPD in the PCV-13 period. We estimated a 85 (95\% Cl, 64\%-95\%) reduction in the incidence of the six additional PCV13-serotypes and 74\% (95\% $\mathrm{CI}, 63 \%-82 \%)$ for IPD caused by all serotypes in children <2 years. We observed a $28 \%(95 \% \mathrm{Cl}, 18 \%-27 \%)$ reduction in IPD-attributable 30-day mortality, from 3.4 deaths $(95 \% \mathrm{Cl}, 3.2-3.6)$ to $3.1(95 \% \mathrm{Cl}, 2.8-3.4)$ and $2.4(95 \% \mathrm{Cl}$, 2.2-2.7) per 100.000 population in the PCV7 and PCV13-periods. The decline in mortality was observed across all age groups, but mainly in the non-vaccinated population. For serotypes 1 and 3 , there were no significant changes in incidence beyond what would be expected from the natural cyclical patterns. There was a significant increase in 19A-incidence following PCV7, but by 2012, the incidence declined towards baseline levels.

Conclusion: We observed a further decline on the incidence of IPD shortly after the shift from PCV-7 to PCV-13 in the national immunization programme. There was a substantial population-level decline in pneumococcalattributable mortality of nearly $30 \%$ among non-vaccinated persons.

Conflict of interest 
ISPPD-0182

Controlling Pneumococcal Disease Around the Globe

\section{NEAR ELIMINATION OF OTITIS MEDIA (OM) CAUSED BY THE PCV13 SEROTYPES, SHORTLY AFTER SEQUENTIAL INTRODUCTION OF PCV7/PCV13 IN ISRAEL}

S. Ben-Shimol ${ }^{1}$, N. Givon-Lavi ${ }^{1}$, E. Leibovitz ${ }^{1}$, D. Greenberg ${ }^{1}$, R. Dagan ${ }^{1}$

${ }^{1}$ Pediatric Infectious Disease Unit, Soroka University Medical Center and Ben-Gurion University of the Negev, Beer-Sheva, Israel

Background and Aims: Before PCV introduction, PCV7/PCV13 serotypes constituted 50\%/ 80\% of all pneumococcal middle ear fluid (MEF) isolates. We assessed pneumococcal OM (Pnc-OM) incidence in children $<24$ mo during Jul 2004 - June 2013.

Methods: Our medical center serves a captive population of $\sim 30,000$ children $<24$ months. PCV7 and PCV13 were introduced in Jul 2009 and Nov 2010, respectively. 70\% of children <24mo received $\geq 2$ PCV7 and PCV13 doses in Apr 2010 and Apr 2012, respectively. We defined 3 sub-periods: Pre-PCV (Jul 2004 - Jun 2008), PCV7 (Jul 2010 - Jun 2011) and PCV13 (Jul 2012 - Jun 2013). Populations at risk and culture methods were described before (Dagan, 49th IDSA, Abst. 1343).

Results: Overall, 1,893 Pnc-OM episodes were identified. $\geq 1$ factors associated with complicated OM was present in $61.3 \%$ of episodes. During the PCV7 and PCV13 periods, reduction of $73 \%$ and $96 \%$, respectively, was observed in OM caused by PCV7+6A serotypes vs. pre-PCV period (Figure/Table). Serotypes 1, 3, 5, 7F, 19A (grouped) incidence increased by $25 \%$ in the PCV7 period, but decreased by $85 \%$ in the PCV13 period. No significant increase in nonPCV13 OM was observed. This resulted in 31\% and 77\% reduction of Pnc-OM in the PCV7 and the PCV13 periods, respectively.

Conclusion: A rapid 2-step reduction in Pnc-OM in children $<2$ years was observed after PCV7/PCV13 introduction.

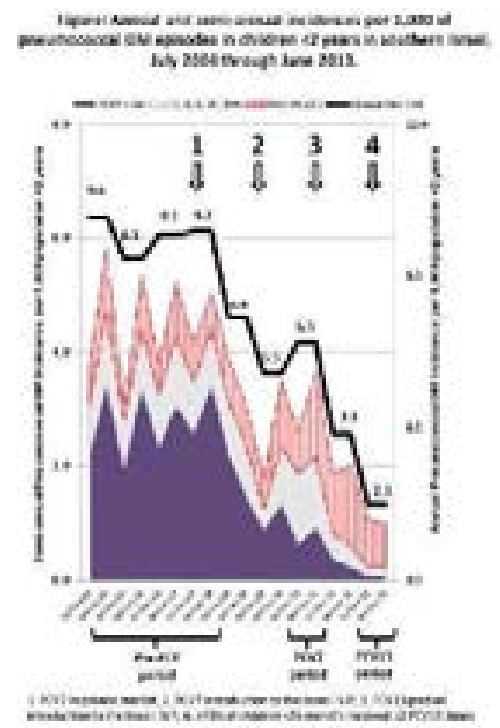

\begin{tabular}{|c|c|c|c|c|c|}
\hline & 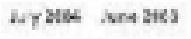 & 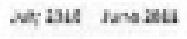 & 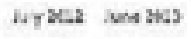 & messes & wapeco \\
\hline & 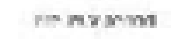 & swrent & Fristian & 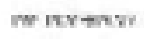 & 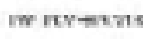 \\
\hline 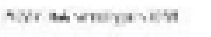 & 55204 & is & 23 & c.estas. & $200 ; 236360$ \\
\hline 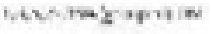 & 22,63 & 25 & 23 & $+E\{643-4.7 \%$ & $3: 5 ; 2 \pi n-230$ \\
\hline mareson & $25 \pi 02$ & $\Delta$ & 29 & $3.4755>2141$ & $\therefore 00 \cos 200$ \\
\hline 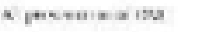 & $21=0$ & 63 & 21 & C.E 1653 C.AH & $320 ; 2.30230$ \\
\hline
\end{tabular}

Conflict of interest 
ISPPD-0183

Controlling Pneumococcal Disease Around the Globe

\section{SEROTYPE-SPECIFIC PCV7/PCV13 EFFECT ON INVASIVE PNEUMOCOCCAL DISEASE (IPD) IN CHILDREN <5 YEARS IN ISRAEL, JULY 2004 - JUNE 2013}

S. Ben-Shimol ${ }^{1}$, D. Greenberg ${ }^{1}$, N. Givon-Lavi ${ }^{1}$, R. Dagan $^{1}$

${ }^{1}$ Pediatric Infectious Disease Unit, Soroka University Medical Center and Ben-Gurion University of the Negev, Beer-Sheva, Israel

Background and Aims: Seven-valent pneumococcal conjugate vaccine (PCV7) Introduction to the Israeli national immunization plan (NIP) in Jul-2009 resulted in a fast reduction of PCV7 serotype-specific IPD rate. Since Nov-2010, PCV13 gradually replaced PCV7 without catch-up. We assessed PCV7/PCV13 impact on serotype-specific IPD in children $<5$ years in Israel.

Methods: Methods were previously described (Ben-Shimol et-al, 50th IDSA, Abstr. 442, 2013).

Results: Overall-IPD incidence (per 100,000 children $<5$ years) declined by $63 \%$, from a mean of $50.6 \pm 2.3$ in the pre-PCV period (Jul-2004 through Jun-2008) to 18.8 in 2012-2013. (Figure and Table) Comparing the last study year (Jul-2012 through Jun-2013) to the pre-PCV period: PCV7+6A serotypes disease incidences declined by $\geq 91 \%$; the 5 additional PCV13 serotypes (PCV5) decreased significantly by 70\%; single serotypes 1, 5 and $19 \mathrm{~A}$ decreased significantly, while serotypes 7F and 3 IPD were not significantly reduced. Non-PCV13 serotypes IPD rates increased overall by $140 \%(p<0.001)$.

Conclusion: Substantial and significant reduction of overall IPD and IPD caused by 10 of PCV13 serotypes was observed shortly after PCV13 introduction to NIP. Disease caused by serotypes 3,4 and 7F was rare and was not significantly reduced. Non-PCV13 IPD increased, but this had only moderate effect on overall-IPD.

Figure: Annual serotype-specific IPD rates in children <5 years old, Israel (July 2004 - June 2013)
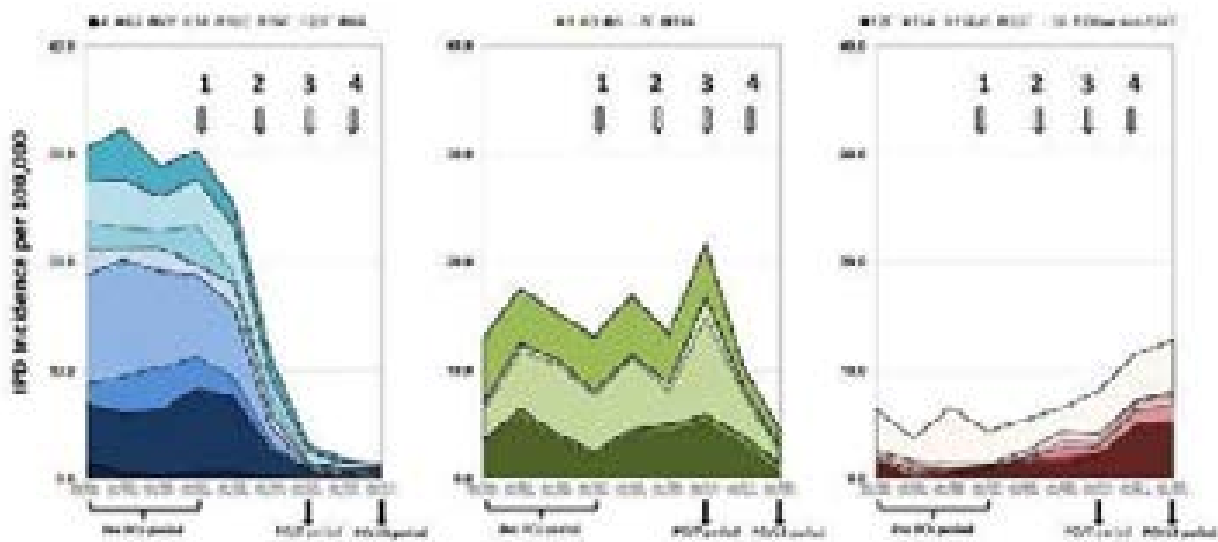

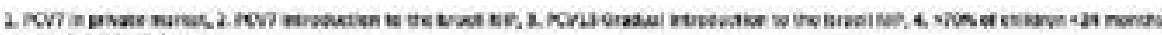
rewenterevisiders

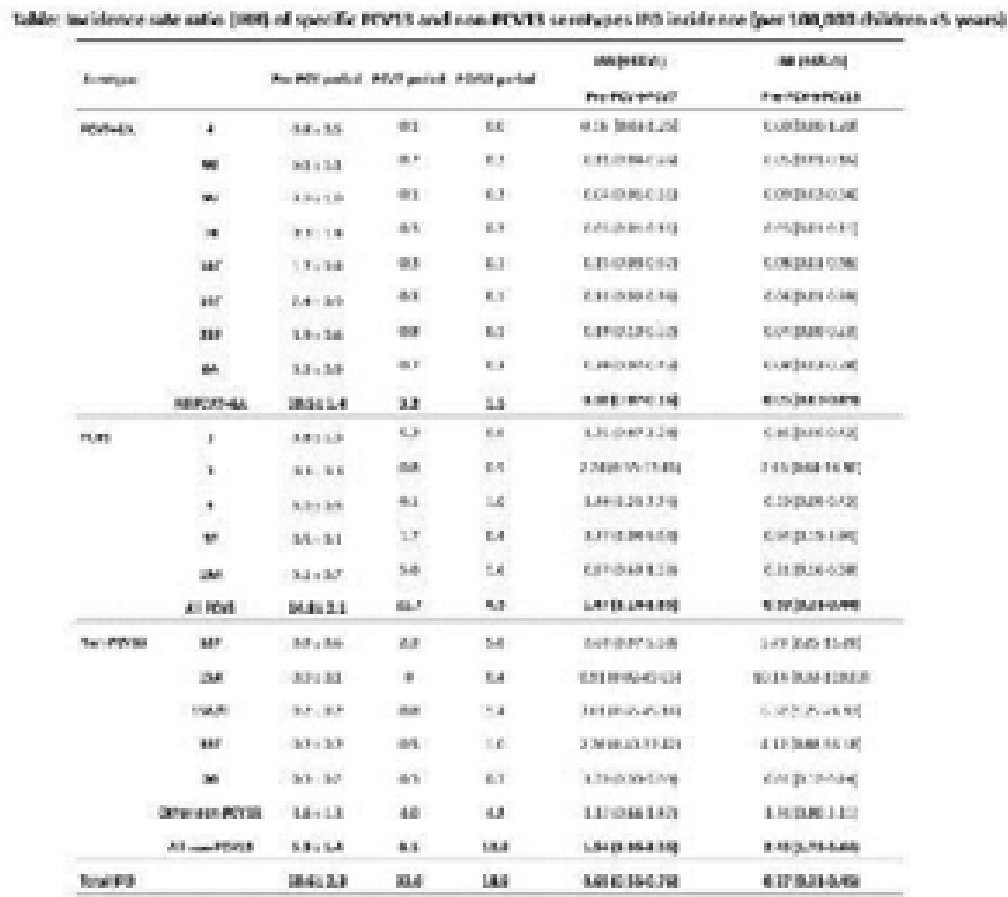


ISPPD-0520

Controlling Pneumococcal Disease Around the Globe

\title{
MATERNAL IMMUNIZATION WITH PNEUMOCOCCAL POLYSACCHARIDE VACCINE: PERSISTENCE OF MATERNAL ANTIBODIES IN INFANTS
}

\author{
E. Berezin ${ }^{1}$, C. Lopes ${ }^{2}$, D. Goldblatt ${ }^{3}$, L. Ashton ${ }^{3}$, M.R. Cardoso ${ }^{4}$ \\ ${ }^{1}$ Pediatrics, Faculdade de Ciências Médicas da Santa Casa de São Paulo, São Paulo, Brazil; ${ }^{2}$ Pediatrics, Santa Casa, São Paulo, Brazil \\ ${ }^{3}$ Imunology, Institute of Child Health, London, United Kingdom; ${ }^{4}$ Epidemiology, Faculdade de Saúde Pública Universidade de S. Paulo, São Paulo, \\ Brazil
}

Objective: To evaluate the level and the persistence of maternal antibodies in infants after maternal immunization with pneumococcal polysaccharide vaccine.

Methods: A total of 100 pregnant women without relevant obstetric clinical conditions was included in the study between May 2005 and January 2006. The pregnant women were assigned to two groups, based on a randomized list during routine low-risk pre-natal visits. The first Group (VAC) received the $23 \mathrm{~V}$ non-conjugate pneumococcal polysaccharide vaccine (Pn23V) shortly after enrolment (pregnant women at 28 weeks or later) and the second Group (NO VAC) received no vaccine to investigate the antibody persistence, we collected blood samples from the mothers after 1 month of delivery and from the infants at 1 month and 6 months of age. Antibody titers were measured for serotypes 1,6B and 14.

Results: Mother's immunization induced antibody responses to all serotypes tested. GMC's of specific IgG were significantly higher in the vaccinated group compared to unvaccinated controls for all 3 serotypes tested. A significantly higher proportion of vaccinated mothers achieved protective concentrations of IgG to serotype 1 compared to unvaccinated controls. However, proportions above both 0.35 and $1.0 \mu \mathrm{g} / \mathrm{ml}$ were relatively high for serotypes $6 \mathrm{~B}$ and 14 in unvaccinated mothers. For the infants at one month of age, GMC's and proportions above 0.35 and $1.0 \mu \mathrm{g} / \mathrm{ml}$ were significantly higher in the infants born from mothers who received vaccine compared to infants born from unvaccinated mothers. At one month of age infant IgG to serotypes $1,6 \mathrm{~B}$ and 14 were $19.6 \%$, $20.8 \%$ and $20.8 \%$ of the maternal levels. By 6 months of age antibody levels had dropped by $>90 \%$ to all 3 serotypes. Despite this rapid decline in antibody, at 6 months of age proportions $>0.35 \mu \mathrm{g} / \mathrm{ml}$ remained higher in the infants of vaccinated mothers than controls for all three serotypes although proportions $>1.0 \mu \mathrm{g} / \mathrm{ml}$ were not significant for any serotype.

No conflict of interest

ISPPD-0127

Controlling Pneumococcal Disease Around the Globe

\section{IMMUNOGENICITY AND SAFETY OF 13-VALENT PNEUMOCOCCAL CONJUGATE VACCINE IN HIV INFECTED CHILDREN AND ADULTS NOT PREVIOUSLY IMMUNIZED WITH PNEUMOCOCCAL VACCINE}

A. Bhorat ${ }^{1}$, S. Madhi' ${ }^{2}$, F. Laudat ${ }^{3}$, V. Sundaraiyer ${ }^{4}$, A. Gurtman ${ }^{3}$, K. Jansen ${ }^{3}$, D. Scott ${ }^{3}$, E. Emini ${ }^{3}$, W. Gruber ${ }^{3}$, B. Schmoele-Thoma ${ }^{3}$

${ }^{1}$ Family Physician, Soweto Clinical Trials Centre, Johannesburg, South Africa; ${ }^{2}$ Department of Science/National Research Foundation, University of Witwatersrand, Johannesburg, South Africa; ${ }^{3}$ Vaccine Research, Pfizer Vaccine Research, Collegeville, USA; ${ }^{4}$ Statistics, InVentiv Health Clinical LLC, Princeton, USA

Background and Aims: HIV-infected individuals, including those on highly active antiretroviral therapy (HAART), are at increased risk of pneumococcal disease. This open-label, single-arm study assessed safety, tolerability, and immunogenicity of 13-valent pneumococcal conjugate vaccine (PCV13) followed by 23-valent polysaccharide vaccine (PPSV23) in pneumococcal vaccine-naive, HIV-infected subjects.

Methods: Subjects aged $\geq 6$ years received 3 PCV13 doses followed by 1 PPSV23 dose at 1 month intervals. Blood samples were obtained before and 1 month after each vaccination; serotype-specific anti-capsular IgG and antipneumococcal opsonophagocytic activity (OPA) were measured. Local and systemic reactions were collected for 14 days after PCV13; adverse events, graded for severity, relation to PCV13, and seriousness, were collected for 6 months after PPSV23.

Results: 150 subjects aged $6-17$ years and 151 subjects $\geq 18$ years were enrolled and vaccinated; $97 \%$ were on HAART; $71 \%$ had viral loads $<50$ copies/mL. Mean CD4 count was $717 \pm 381$ cells $/ \mu \mathrm{L}$. After one PCV13 dose, IgG geometric mean fold-rises (GMFRs) and OPA GMFRs were 1.15-15.79 and 4.8-89.4, respectively, in 6-17-year-olds and 1.62-8.29 and 4.1-52.2, respectively, in $\geq 18$-year-olds. OPA GMFRs were similar or higher following doses 2 and 3; IgG GMFRs were mostly higher in the younger age group. Injection site pain (mostly mild to moderate) was the most frequent local reaction. There were no PCV13-related serious adverse events.

Conclusion: PCV13 appeared safe and immunogenic in pneumococcal vaccine-naive, HIV-infected subjects aged $\geq 6$ years with CD4 cell counts $\geq 200$ cells/ $\mu \mathrm{L}$. Vaccine serotype-specific increases in OPA were observed after one PCV 13 dose; and similar or higher after subsequent PCV13 doses.

Conflict of interest 
ISPPD-0031

Controlling Pneumococcal Disease Around the Globe

\section{EFFECT OF PCV1O VACCINATION ON PNEUMOCOCCAL SEROTYPES IN BRAZIL USING THE NATIONAL PNEUMOCOCCAL LABORATORY NETWORK SURVEILLANCE}

M.C.C. Brandileone ${ }^{1}$, S.C.G. Almeida ${ }^{1}$, R.C. Zanella ${ }^{1}$, M.L.L.S. Guerra ${ }^{1}$, S. Bokermann ${ }^{1}$, L.S. Prado ${ }^{1}$, L.L. Silva ${ }^{1}$, A.L. Andrade $^{2}$

${ }^{1}$ Bacteriology, Adolfo Lutz Institute, São Paulo, Brazil; ${ }^{2}$ Institute of Tropical Pathology and Public Health, Federal University of Goiás, Goiânia, Brazil

Background and Aims: In 2010, Brazil introduced the PCV10 into the National Immunization Program in a $3+1$ dose schedule (2,4,6 and 12-18m of age), and in a catch-up campaign for children aged $12-<24 \mathrm{~m}$. In $2011 / 2012$, the PCV10 coverage for $<1$ y old was $\sim 80 \%$. After two years of PCV10-introduction, we investigated the vaccination effect on the PCV10-serotypes in vaccinated and unvaccinated population.

Methods: We used data from the national Streptococcus pneumoniae laboratory based surveillance. The mean number of PCV10-types and non-vaccine types were compared between the pre- (Jan/2008-Dec/2009) and post(Jan/2011-Dec/ 2012) vaccination periods and used to assess the PCV10-effectiveness (1-Odd ratio).

Results: During 2008-2012, 2,399 invasive S. pneumoniae were studied. Annual number of PCV10-types in preand post-vaccination by age group is shown in table 1. PCV10-types were significantly less prevalent in the postvaccination period compared to the pre-vaccination for all age groups.

Table 1. Comparison of PCV10-types between pre- and post-PCV10 vaccination

\begin{tabular}{|c|c|c|c|c|c|c|}
\hline \multirow[b]{2}{*}{ Age group } & \multicolumn{2}{|c|}{ Pre-PCV10 } & \multicolumn{2}{|c|}{ Post-PCV10 } & \multirow{2}{*}{$\begin{array}{l}\text { PCV10 types } \\
\text { Post- vs Pre-PCV10 } \\
\text { p-value }\end{array}$} & \multirow{2}{*}{$\begin{array}{l}\text { PCV10 } \\
\text { Effectiveness }\end{array}$} \\
\hline & $\begin{array}{l}\text { *No. } \\
\text { PCV10 } \\
\text { types }\end{array}$ & *No.Total & $\begin{array}{l}\text { *No. } \\
\text { PCV10 } \\
\text { types }\end{array}$ & *No.Total & & \\
\hline$<2 y$ & 131 & 169 & 43 & 125 & $<0.005$ & $85 \%$ \\
\hline $2-<5 y$ & 58 & 78 & 42 & 70 & 0.048 & $50 \%$ \\
\hline $5-49 y$ & 180 & 341 & 156 & 390 & 0.005 & $40 \%$ \\
\hline $50-64$ y & 38 & 82 & 50 & 160 & 0.021 & $47 \%$ \\
\hline$\geq 65$ y & 24 & 60 & 41 & 112 & 0.662 & --- \\
\hline
\end{tabular}

*Annual media of number of isolates

Conclusion: Two-years after PCV10 introduction, vaccine-serotypes reduced significantly in vaccinated population and a significant herd effect was observed in all age-group but $\geq 65 \mathrm{y}$ old.

Acknowledgment: SIREVAII-PAHO for supplying the pneumococcal antisera; CNPQ for grant support to MCCBrandileone(\#302175/2010-5) and ALA(\#306096/2010-2).

No conflict of interest

\section{CLINICAL MANIFESTATIONS OF INVASIVE PNEUMOCOCCAL DISEASE CAUSED BY VACCINE AND NON-VACCINE TYPE} STRAINS IN CHILDREN AND ADULTS

S. Browall ${ }^{1}$, E. Backhaus ${ }^{2}$, P. Naucler ${ }^{3}$, I. Galanis ${ }^{1}$, K. Sjöström ${ }^{1}$, D. Karlsson ${ }^{4}$, S. Berg ${ }^{5}$, J. Luthander ${ }^{6}$, M. Eriksson ${ }^{6}$, C. Spindler ${ }^{7}$, M. Ejdebäck ${ }^{4}$, B. Trollfors ${ }^{5}$, M. Kalin ${ }^{7}$, R. Andersson ${ }^{8}$, B. Henriques-Normark ${ }^{1}$

${ }^{1}$ Department of Microbiology Tumor and Cell Biology, Karolinska Institutet, Stockholm, Sweden; ${ }^{2}$ Department of Infectious Diseases,

Skaraborg Hospital, Skövde, Sweden; ${ }^{3}$ Department of Infectious Diseases, Karolinska Institutet, Stockholm, Sweden; ${ }^{4}$ School of Life Sciences, University of Skövde, Skövde, Sweden; ${ }^{5}$ Queen Silvia Children's Hospital, Sahlgrenska University Hospital, Gothenburg, Sweden; ${ }^{6}$ Department of Paediatrics, Karolinska University Hospital, Stockholm, Sweden; ${ }^{7}$ Department of Infectious Diseases, Karolinska University Hospital, Stockholm, Sweden; ${ }^{8}$ Department of Infectious Diseases Institute of Biomedicine, Gothenburg University, Gothenburg, Sweden

Pneumococcal conjugated vaccines (PCVs) have recently been introduced in the childhood vaccination program in several countries, including Sweden showing a protective effect against invasive disease (IPD) caused by vaccine types, and an increase of non-vaccine type disease in both vaccinated children and non-vaccinated adults. It is not known to what extent non-vaccine serotypes provide the same ability to cause IPD and whether the disease manifestations are the same as for vaccine types. Here we used a large collection $(n=2288)$ of patient data and corresponding pneumococcal isolates from IPD in adults $(n=2096)$ and children $(n=192)$ to explore correlations of clinical manifestations to vaccine and non-vaccine types. Additional 165 invasive and 550 pneumococcal carriage 
isolates from children were used to calculate the invasive disease potential for different serotypes. The odds ratio for causing IPD in children was lower for non-PCV13 strains as compared to vaccine type strains. Children infected with non-PCV13 strains had more underlying diseases and tended to have meningitis more often (47\%) and to have a higher OR for intensive care attendance (33\%) than children infected with PCV13 serotypes (33\% and 23\% respectively). Adult IPD patients infected with non-PCV13 strains showed bacteremia more often, more underlying diseases but less pneumonia, while the OR for meningitis, death and age did not differ between infections with nonPCV13 or PCV13 strains. Childhood vaccination with PCV will likely not change the clinical manifestation of IPD in non-vaccinated adults even though there will be a replacement with non-vaccine type strains due to herd immunity.

No conflict of interest

ISPPD-0023

Controlling Pneumococcal Disease Around the Globe

\section{EPIDEMIOLOGY OF PNEUMOCOCCAL MENINGITIS IN THE METROPOLITAN REGION OF SALVADOR, BRAZIL, BEFORE AND AFTER THE INTRODUCTION OF PCV10}

P.R. Lobo' ${ }^{1}$, J. Azevedo ${ }^{1}$, E. Silva ${ }^{1}$, E.C. Escobar ${ }^{1}$, A.P.O. Menezes ${ }^{1}$, K. Salgado ${ }^{2}$, M.G. Reis ${ }^{1}$, J.N. Reis ${ }^{3}$, L.C. Campos $^{1}$

${ }^{1}$ Laboratorio de Patologia e Biologia Celular, Centro de Pesquisas Goncalo Moniz - FIOCRUZ, Salvador, Brazil; ${ }^{2} \mathrm{Hospital}$ Couto Maia, Hospital Couto Maia, Salvador, Brazil; ${ }^{3}$ Faculdade de Farmacia, Universidade Federal da Bahia, Salvador, Brazil

Background and Aims: 10-valent pneumococcal conjugate vaccine (PCV10) was implemented in Brazil in 2010. The aim of this study was to describe the incidence, clinical and epidemiological characteristics of pneumococcal meningitis and serotype distribution before (January 2008-May 2010) and after (June 2010-December 2012) PCV10 introduction.

Methods: We performed a 5-year (2008-2012) hospital-based surveillance at a reference hospital for infectious diseases in Salvador. All pneumococcal isolates were serotyped by multiplex PCR and/or Quellung method. Results: A total of 104 cases of pneumococcal meningitis were identified. The incidence of pneumococcal meningitis decreased from 0.71 (pre-PCV10) to 0.46 (post-PCV10) cases per 100,000 persons for all age groups ( $p$ $<0.03)$. A significantly decline was observed in children with $<2$ years of age $(p<0.058)$. The overall case-fatality rate decreased from $24 \%$ (pre-PCV10) to $17.6 \%$ (post- PCV10). Overall, the incidence of disease by PCV10 serotypes decreased from 0.31 (pre-PCV10) to 0.15 cases (post-PCV10) per 100,000 persons ( $<0.03$ ). The most prevalent serotypes were $14(14 \%), 23 \mathrm{~F}(10,55), 6 \mathrm{~B}(8.8 \%)$ and $3(7 \%)$ (pre-PCV10) and, $18 \mathrm{C}(11.8 \%), 23 \mathrm{~F}(8.8 \%)$ and $12 \mathrm{~F}$ (8.8\%) (post-PCV10).

Conclusion: There was a decline in the incidence of pneumococcal meningitis after PCV10 introduction. The distribution of serotypes showed a normal fluctuation without predominance of any serotype. Continued surveillance studies will be essential to evaluate the benefits of the vaccination and serotype dynamics in Brazil. Acknowledgments: Conselho Nacional de Desenvolvimento Científico e Tecnológico (\#483674/2010-9), Programa Estratégico de Apoio à Pesquisa em Saúde (PAPES \#407551/2012-3) and Fundação Oswaldo Cruz.

No conflict of interest

ISPPD-0285

Controlling Pneumococcal Disease Around the Globe

\section{INVASIVE PNEUMOCOCCAL SEROTYPE DISTRIBUTION IN THE PHILIPPINES}

M.R. Capeding ${ }^{1}$, L. Sombrero ${ }^{1}$, P. Retiban ${ }^{1}$

${ }^{1}$ Microbiology, Research Institute for Tropical Medicine, Muntinlupa City, Philippines

Background and Aims: Several hospital and community based studies on invasive pneumococcal disease (IPD) had been conducted for the past 20 years. In all the studies Streptococcus pneumoniae was one of the most prevalent etiologic agents. Only serogrouping was performed among isolates. This study aims to describe the serotype distribution, age distribution and antibiotic resistance patterns of S. pneumoniae isolates from hospitalized patients. Methods and Results: This study was conducted from June 2010 to September 2012. A total of 301 S. pneumoniae isolates were confirmed from 311 sample isolates collected from 42 tertiary hospital laboratories nationwide. These were collected from blood, CSF, pleural fluid and peritoneal fluid. The age range of patients were from 2 days to 87 years old. Pediatric patients comprised $41.5 \%$ whereas adult patients $56.4 \%$. Serotype distribution of $S$. pneumoniae and vaccine coverage for PCV13 for all age groups $68.4 \%$; Pediatrics $71.2 \%$ and adult age group $65.5 \%$. Antibiotic resistance patterns of $S$. pneumoniae from hospitalized patients was significantly low except resistance to co-trimoxazole.

Conclusion: Information on the serotypes distribution included in this study has implications for future pneumococcal immunization program in the country.

No conflict of interest 
ISPPD-0042

Controlling Pneumococcal Disease Around the Globe

\section{REDUCTION OF PNEUMOCOCCAL INVASIVE DISEASE, DISAPPEARANCE OF SEROTYPE 19-A AND PNEUMOCOCCAL MENINGITIS FOLLOWING 13-VALENT PNEUMOCOCCAL CONJUGATED VACCINE IN MEXICAN CHILDREN}

E. Chacon-Cruz ${ }^{1}$, A. Alvelais-Arzamendi ${ }^{2}$, T. Stevanovic-Mihailovic ${ }^{3}$, R.M. Rivas-Landeros ${ }^{4}$, M.L. Volker-Soberanes ${ }^{4}$, O. Armenta-Llanes ${ }^{5}$

${ }^{1}$ Pediartric Infectious Diseases, Hospital General de Tijuana, Tijuana, Mexico; ${ }^{2}$ Pediartrics, Hospital General de Tijuana, Tijuana, Mexico;

${ }^{3}$ School of Medicine, University of Xochicalco, Tijuana, Mexico; ${ }^{4}$ Microbiology, Hospital General de Tijuana, Tijuana, Mexico; ${ }^{5}$ Pediatrics, Hospital General de Tijuana, Tijuana, Mexico

Background and Aims: The Tijuana, Mexico and San Diego, California, is the world's most transited frontier. We have previously published and presented pneumococcal serotype replacement following 7-valent pneumococcal conjugated vaccine, especially by serotypes 19-A, 3 and 6A/C. Since May/2012 universal vaccination with the 13-valent pneumococcal conjugated vaccine (PCV-13) was introduced for all children. This study analyzes the effectiveness of PCV-13 following 16 months after PCV-13 implementation in the region.

Methods: Since October/2005 until September/2013 (8 years) we underwent an active surveillance for all Invasive Pneumococcal Diseases (IPD) in children $<16$ years admitted at the Tijuana General Hospital. Only cultureconfirmed cases were included. Following identification, serotyping was performed using the Quellung Reaction (Statens Serum Institute ${ }^{\circledR}$ Copenhagen, Denmark). Descriptive analysis was performed using Excel ${ }^{\circledR}$.

Results: 48 cases of confirmed IPD were found. Clinical diagnosis were pleural empyemas (48\%), sepsis with/ without other conditions (27\%), meningitis (25\%), otomastoiditis (18.75\%) and bacteremic pneumonia (4.15\%). From eight confirmed IPD cases during 2011-12, there were only two cases during $2012-13$ (75\% reduction in overall IPD's following PCV-13 introduction). From an average of 1.75 yearly cases due to pneumococcal serotype 19-A, and pneumococcal meningitis in the last three years, there were no cases of neither serotype 19-A S. pneumoniae associated IPD's and pneumococcal meningitis during 2012-13.

Conclusion: This is the first Mexican study based on active surveillance, and second in Latin America, that shows early findings of effectiveness of PCV-13 on reduction of overall IPD, and disappearance of pneumococcal serotype 19-A and pneumococcal meningitis in children.

No conflict of interest

ISPPD-0257

Controlling Pneumococcal Disease Around the Globe

\section{LONG TERM FEEDING OF O-3 PUFA PROVIDES ADDITIONAL ADVANTAGE TO BALB/C MICE UPON INFECTION WITH STREPTOCOCCUS PNEUMONIAE}

S. Chhibber ${ }^{1}$, A. Saini ${ }^{2}$, K. Harjai ${ }^{3}$

${ }^{1}$ Microbiology, Panjab University Chandigarh, Chandigarh, India; ${ }^{2}$ Microbiology, National Institute of Immunology, New Delhi, India; ${ }^{3}$ Microbiology, Panjab University, Chandigarh, India

Aims and Background: In the present study, effect of extended feeding of flaxseed and sea-cod oil and their effect on lung infection by Streptococcus pneumoniae was evaluated.

Methods: Animals were given flaxseed and sea-cod oil for a period of 90 days enterally and challenged intra-

tracheally with S. pneumoniae D39 serotype2. Survival of animals and various inflammatory parameters i.e. myeloperoxidase, malondialdehyde, nitric oxide, leukotriene B4, cytokines (IL-1 3 , TNF- $\alpha$, IL-10), ICAM-1 levels and fatty acid analysis in the lung homogenates were determined.

Results: Increased survival of mice accompanied with reduction in bacterial load and inflammatory parameters was observed. Maximum decrease in lung bacterial load was observed in sea-cod fed animals. Pathological evaluation of lung tissue showed that supplementation of diet with $\omega-3$ PUFA oils resulted in lowering the severity of infection from severe pneumonia to moderate pneumonia.

Conclusion: The results showed that 90 days feeding with these PUFA oils protected the animals from developing severe pneumonia as compared to control (normal saline fed) animals.

Significance and Impact of Study: In a recent study from our laboratory we have demonstrated that length of PUFA feeding has an impact on lung colonization by S. pneumoniae. The present study supports our earlier finding that extended feeding is beneficial to the host.

No conflict of interest 
ISPPD-0152

Controlling Pneumococcal Disease Around the Globe

\section{RAPID DECREASE OF INVASIVE PNEUMOCOCCAL DISEASE AFTER ONE YEAR OF THE INTRODUCTION OF PCV13 IN ARGENTINA}

D.L. Lazzarini ${ }^{1}$, M.M. Contrini ${ }^{1}$, E. Glatstein ${ }^{2}$, A.V. Garnero ${ }^{2}$, G.C. Ezcurra ${ }^{3}$, E. Teplitz ${ }^{4}$, M. lácono ${ }^{5}$, M. Gamarra ${ }^{1}$, A. Procopio $^{1}$, M.S. Vázquez ${ }^{1}$, E.L. López ${ }^{1}$

${ }^{1}$ Infectious Diseases, Hospital de Niños “Ricardo Gutiérrez", Buenos Aires, Argentina; ${ }^{2}$ Infectious Diseases, Hospital de Niños "Santísima Trinidad", Córdoba, Argentina; ${ }^{3}$ Infectious Diseases, Hospital de Niños “Orlando Alasia”, Santa Fe, Argentina; "Infectious Diseases, Hospital Italiano, Bahía Blanca, Argentina; ${ }^{5}$ Infectious Diseases, Hospital Provincial, Neuquén, Argentina

Background: Universal pneumococcal vaccination (UPV) in Argentina began in Jan 2012 using $2+1$ schedule for children $(\mathrm{CH})<1$ y old and catch-up with 2 doses for $\mathrm{CH} 12$ to 24 mo.

Objective: To evaluate the impact of PCV13 IP incidence in 2012 vs pre-vaccination period (2009-2011).

Methods: Multicenter, prospective study. Hospitalized $\mathrm{CH}<60$ mo with confirmed IPD and/or consolidated pneumonia (CP)(WHO criteria) were included. Patients (pts) with CP or empyema (E) confirmed by any other agent than S. pneumoniae were excluded. Demographic/clinical data recorded. Stata v11.2 was used.

Results: Pts included 1303; 1003 (77\%) CP, of them 56/939 (5,9\%) were confirmed as pneumococcal consolidated pneumonia (P-CP); E: 181 (13,8\%), 65/181 (35,9\%) confirmed as pneumococcal empyema (PE) by blood and/or pleural fluid culture; Meningitis (M) 28 (2,1\%); other IPD: 91 (6,98\%), including bacteremia, peritonitis, arthritis $\mathrm{CP}$ was more freq in $\mathrm{CH}<1$ y $(613$ vs $390, \mathrm{p}=0.0001$; in pts $>24$ mo $(112$ vs $69, \mathrm{p}<0.0001)$ and $64 \%$ of $\mathrm{M}$ occurred in $\mathrm{CH}<12$ mo. The decrease of IPD, CP and Pneumococcal Pulmonary Disease (PPD) (defined as P-CP + PE), during 2012 is shown.

\begin{tabular}{|c|c|c|c|c|c|c|c|}
\hline \multirow[b]{2}{*}{ Year } & \multirow[b]{2}{*}{ Discharge } & \multicolumn{2}{|c|}{ Global IPD } & \multicolumn{2}{|l|}{ CP } & \multicolumn{2}{|c|}{ PPD (P-CP + PE) } \\
\hline & & $\begin{array}{l}\text { No. } \\
\text { cases }\end{array}$ & Rate* $(95 \% \mathrm{Cl})$ & $\begin{array}{l}\text { No. } \\
\text { cases }\end{array}$ & Rate* $(95 \% \mathrm{Cl})$ & $\begin{array}{l}\text { No. } \\
\text { cases }\end{array}$ & $\begin{array}{l}\text { Rate* } \\
(95 \% \mathrm{Cl})\end{array}$ \\
\hline 2009-2011 & $\begin{array}{l}34,038 \\
\text { (average) }\end{array}$ & 48 & $\begin{array}{r}14.1 \\
(10.4-18.7) \\
\end{array}$ & 284 & $\begin{array}{r}83.4 \\
(74.0-93.7) \\
\end{array}$ & 34 & $\begin{array}{r}9.9 \\
(6.9-13.9) \\
\end{array}$ \\
\hline 2012 & 32,100 & 26 & $\begin{array}{r}8.1 \\
(5.3-11.9) \\
\end{array}$ & 152 & $\begin{array}{r}47.3 \\
(39.8-54.9) \\
\end{array}$ & 18 & $\begin{array}{r}5.6 \\
(3.3-8.8) \\
\end{array}$ \\
\hline Decrease $p$ & & $\begin{array}{r}-43 \% \\
0.02\end{array}$ & & $\begin{array}{r}-43.3 \% \\
<0.001\end{array}$ & & $\begin{array}{r}-47 \% \\
0.04\end{array}$ & \\
\hline
\end{tabular}

* Rates/10,000 hospitalizations. No significative difference in rates between yr in prevaccination period was observed.

Conclusion: 1.A rapid and significative decrease of IPD and PPD after 1 yr of PCV13 introduction was observed. 2. As 2012 was a transitional period, IPD surveillance should be continued to confirm effectiveness of PCV13 in CH. 3 To our knowledge this is the first multicenter study in Latin American countries on PCV13 effectiveness. These preliminary results should encourage the introduction of UPV in developing countries

Conflict of interest

ISPPD-0497

Controlling Pneumococcal Disease Around the Globe

\section{GENETIC DIVERSITY OF STREPTOCOCCUS PNEUMONIAE BELONGING TO NON-VACCINE SEROTYPES}

G.R. Cunha ${ }^{1}$, G.G. Vieira1 ${ }^{1}$, M.P. Mott ${ }^{2}$, T.C.A. Pinto ${ }^{3}$, J. Caierão ${ }^{1}$, P. Hawkins ${ }^{4}$, P.A. d'Azevedo ${ }^{1}$, L.M. Teixeira ${ }^{3}$, L. McGee $^{5}$, C.A.G. Dias ${ }^{1}$

${ }^{1}$ Gram Positive Cocci Laboratory, Ufcspa, Porto Alegre, Brazil; ${ }^{2}$ Microbiological Analysis, Ciscmpa, Porto Alegre, Brazil; ${ }^{3}$ Medical Microbiology, Ufrj, Rio de Janeiro, Brazil; ${ }^{4}$ Microbiology, Emory University, Atlanta, USA; ${ }^{5}$ Microbiology, Centers for Disease Control and Prevention, Atlanta, USA

Background and Aims: PCV10 was introduced in Brazil in 2010. This study aimed to analyze the genetic diversity of the most prevalent isolates belonging to non-vaccine serotypes in Porto Alegre, Southern Brazil.

Methods: Pneumococci were characterized using multilocus sequence typing (MLST), multiple-locus variable number (MLVA) and pulsed-field gel electrophoresis (PFGE). Seventy isolates (24 pre- and 46 post-vaccination) recovered from invasive sites between 2007 to 2013 were evaluated. Mean age of patients was 49.1 years old, with $11.4 \%(8 / 70) \leq 5$ years old and $35.7 \%(25 / 70) \geq 65$ years old. Based on trends to increase after PCV10 (previous data), it were selected the following serotypes: [3 $(n=25), 6 A(n=5), 6 C(n=3), 8(n=13), 12 F(n=13)$ and 19A $(n=11)$ ]. Results: Serotypes 12F [ST218 (13/13)] and 8 [ST53 (11/13); ST404 and ST 8885 (one isolate each)] were highly clonal by MLST and it was confirmed by PFGE. However, MLVA was able to characterize subgroups within STs. Serotypes 3 and 19A were more heterogeneous. ST180 was the most common ST among serotype 3 (72\%) and MLVA characterized 3 major subgroups. Among serotype 19A, ST2878 was the most common ST (4/11), followed by ST320 (2/11). Three pneumococci from ST2878 shared at least $87 \%$ similarity according to MLVA, while the two ST320 pneumococci were $85 \%$ similar. Serotypes $6 \mathrm{~A}$ and $6 \mathrm{C}$ were highly diverse with only the occurrence of 
singletons (MLST); no major groups were observed by MLVA and PFGE.

Conclusion: Our results suggest that MLVA was more discriminatory than MLST and PFGE. This is the first evaluation of the population biology of non-vaccine serotypes in Brazil.

No conflict of interest

ISPPD-0175

Controlling Pneumococcal Disease Around the Globe

\section{INVASIVE PNEUMOCOCCAL DISEASE IN CHILDREN: LATE IMPACT OF PCV7 AND PCV13 VACCINE IN BELGIUM}

I. De Schutter ${ }^{1}$, J. Verhaegen ${ }^{2}$, S. Blumental ${ }^{3}$, C. Genin ${ }^{4}$, M. Hainaut ${ }^{5}$, G. Hanquet $^{6}$, P. Lepage ${ }^{3}$, J. Levy ${ }^{5}$, A. Malfroot $^{7}$, P. Philippet ${ }^{4}$, M. Proesmans ${ }^{8}$, J. Ramet ${ }^{9}$, P. Schelstraete ${ }^{10}$, B. Swennen ${ }^{11}$, D. Tuerlinckx $^{12}$, G. Top $^{13}$, S. Van Lierde ${ }^{14}$, A. Vergison ${ }^{3}$, M. Sabbe 6

${ }^{1}$ Pediatric Pulmonology and Pediatric Infectious diseases, Universitair Ziekenhuis Brussel (UZ Brussel), Brussels, Belgium; ${ }^{2}$ Dept. of Laboratory Medicine-Microbiology Pneumococcal Reference Laboratory, Universitair Ziekenhuis Katholieke Universiteit Leuven, Leuven, Belgium; ${ }^{3}$ Pediatric Infectious Diseases dept., Université Libre de Bruxelles-Hôpital Universitaire des Enfants Reine Fabiola, Brussels, Belgium; ${ }^{4}$ Pediatrics, Clinique de I' Espérance, Liège, Belgium; ${ }^{5}$ Pediatrics, CHU Hôpital St. Pierre, Brussels, Belgium; ${ }^{6}$ Public Health and Surveillance, Scientific Institute of Public Health, Brussels, Belgium; ${ }^{7}$ Pediatric Pulmonology and Pediatric Infectious Diseases, Universitair Ziekenhuis Brussel (UZ Brussel), Brussels, Belgium; ${ }^{8}$ Dept. of Pediatric Pulmonology, Universitair Ziekenhuis Katholieke Universiteit Leuven, Leuven, Belgium; ${ }^{9}$ Pediatrics, Universitair Ziekenhuis Antwerpen, Antwerpen, Belgium; ${ }^{10}$ Pediatric Pulmonology, Universitair Ziekenhuis Gent, Gent, Belgium; ${ }^{11}$ PROVAC, Université Libre de Bruxelles, Brussels, Belgium; ${ }^{12}$ Pediatrics, Cliniques Universitaires UCL Mont-Godinne, Mont-Godinne, Belgium; ${ }^{13}$ Vaccinaties, Vlaams Agentschap Zorg en Gezondheid, Brussels, Belgium; ${ }^{14}$ Pediatrics, Heilig Hart Ziekenhuis, Tienen, Belgium

Background and Aims: In 2007, PCV7 was implemented for infants ( $2+1$ schedule) with a catch-up for $<2$ y olds, and was substituted with PCV13 starting from July 2011. PCV uptake (3rd dose) was estimated at 89-97\% (2012). We present changes in IPD incidence, clinical presentation and serotype distribution during PCV7 implementation (2006-2011) and early impact of PCV13 (2011-2012).

Methods: IPD cases were identified through the national reference centre and paediatrician's surveillance network 'Pedisurv'. Capture-recapture allowed estimating the total number of cases.

Results: Period 2006-2011: IPD-rates decreased in children <2 y from 124.3/100,000 to 90.8/100,000 (-27\%;

$95 \% \mathrm{Cl} 4-44 \%)$, but increased in $2-4$ y olds from $40.5 / 100,000$ to $74.7 / 100,000$ (+83\%, 95\% Cl $25-168 \%)$. IPD-rates attributable to PCV7-serotypes decreased significantly, while non-PCV7-serotype rates increased. In 2-4 y olds, a significant increase in invasive pneumococcal pneumonia was noted from 21.0/100,000 to 47.3/100,000 (+226\%, $95 \% \mathrm{Cl} 135-378 \%$ ), with most common serotypes: 1 (42\%), $5(12 \%)$ and $19 \mathrm{~A}(9 \%)$.

Period 2011-2012: the 6 additional PCV13 serotypes decreased by 31\% (95\%Cl 19-43\%) in children <2 y old (Figure 1).

Conclusion: PCV7 implementation resulted in a significant decrease in IPD in the target group, but simultaneously a significant increase in IPD, mainly pneumonia, was noticed in 2-4 y olds. Based on the preliminary favourable impact of PCV13 introduction, a significant decrease in incidence of IPD and of pneumococcal pneumonia can be expected in the following years.

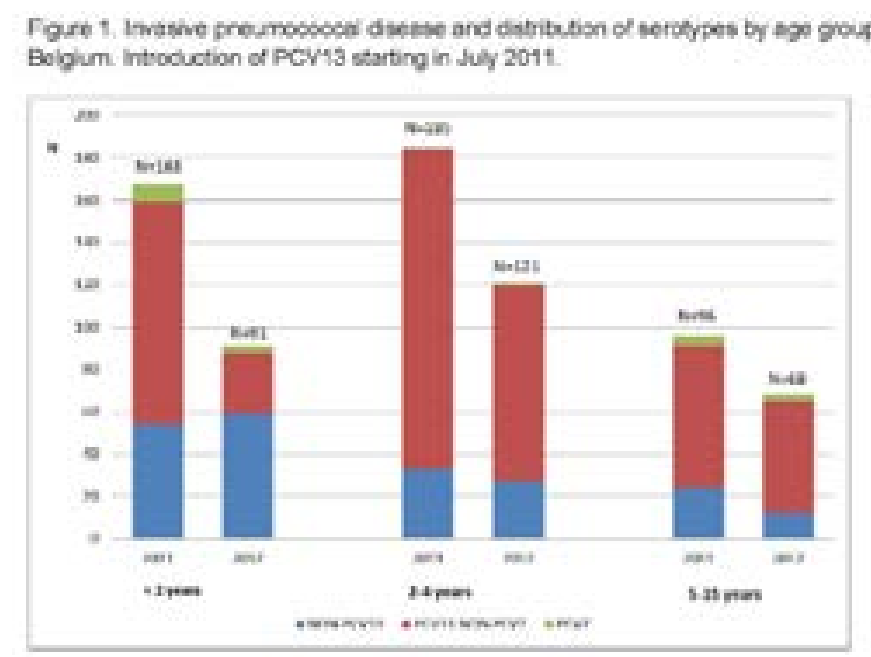

No conflict of interest 


\title{
EFFECTIVENESS OF THREE PNEUMOCOCCAL CONJUGATE VACCINES (PCVs) TO PREVENT INVASIVE PNEUMOCOCCAL DISEASE (IPD) IN QUEBEC, CANADA
}

\section{G. Deceuninck ${ }^{1}$, P. De Wals ${ }^{2}$}

${ }^{1}$ Vaccine Research Unit, Quebec University Hospital Research Centre, Quebec City, Canada; ${ }^{2}$ Social and Preventive Medicine, Laval University, Quebec City, Canada

Background: In Quebec, Canada, a PCV program was implemented in December 2004 and the recommended schedule is $2+1$ doses for low-risk infants. PCV-7 was first used (including catch-up for children up to age 5 years), replaced by PCV-10 in June 2009, and by PCV-13 in January 2011 (no catch-up in both instances). From the beginning, vaccine uptake has been high: $>90 \%$ of children are receiving the recommended number of doses. Objective: To estimate the effectiveness of PCVs.

Methods: IPD cases in children 2-59 months and reported during the years 2005-2012 were eligible and controls randomly identified in the provincial health insurance registry. Parents were interviewed by telephone and immunization records reviewed. Vaccine effectiveness (VE) was computed using logistic regression models. Results: Out of 830 IPD cases reported, full participation was obtained for 480 cases (58\%) and for 1,647 controls. Increased IPD risk was associated with year, colder seasons, younger age, lower maternal education and family income, day-care attendance, comorbidity and influenza immunization whereas PCV vaccination was protective. VE estimates ( $\geq 1$ dose) are shown in the Table $\left({ }^{*} p<0.05\right)$.

\begin{tabular}{|l|l|l|l|}
\hline IPD serotypes & PCV7 & PCV10 & PCV13 \\
\hline PCV7+6A & $90 \% *$ & $100 \%$ & $100 \%$ \\
\hline PCV10+6A & $79 \% *$ & $99 \% *$ & $100 \%$ \\
\hline 19A & $42 \%$ & $67 \% *$ & $69 \%$ \\
\hline PCV13 & $64 \% *$ & $85 \% *$ & $87 \% *$ \\
\hline Non PCV13 & $-13 \%$ & $10 \%$ & $32 \%$ \\
\hline All serotypes & $53 \% *$ & $75 \% *$ & $79 \%$ \\
\hline
\end{tabular}

Conclusion: Results suggest that PCV10 and PCV13 are more effective than PCV7 thanks to the higher number of serotypes, and a high level of cross-protection against 19A for PCV10.

Conflict of interest

ISPPD-0565

Controlling Pneumococcal Disease Around the Globe

\section{FOUR YEARS LABORATORY-BASED SURVEILLANCE OF INVASIVE PNEUMOCOCCAL DISEASES IN MOROCCO: WHAT IMPACTS OF THE PNEUMOCOCCAL CONJUGATE VACCINE IN MOROCCAN POPULATION?}

\author{
I. Diawara ${ }^{1}$, K. Zerouali ${ }^{1}$, H. Belabbes ${ }^{1}$, N. Elmdaghri ${ }^{2}$ \\ ${ }^{1}$ Microbiology, Faculty of medicine and pharmacy - Ibn Rochd Hospital University, Casablanca, Morocco; ${ }^{2}$ Microbiology, Faculty of Medi- \\ cine and Pharmacy - Ibn Rochd Hospital University - Pasteur Institute, Casablanca, Morocco
}

Background and Aims: In October 2010, pneumococcal conjugate 13-valent vaccine (PCV-13) was introduced in Moroccan National Program of Immunization, which was replaced (July 2012) by the PCV-10. We determined the incidence of invasive pneumococcal diseases in Casablanca, Morocco 2 years pre- and 2 years post PCV introduction as well as the rates of antibiotic resistance and serotype distribution.

Methods: This study was conducted at the Ibn Rochd University Hospital of Casablanca, Morocco. Time periods encompassing pre- and post-implementation of PCV (January 2008 to December 2009 and January 2011 to December 2012, respectively) were examined. Serotyping was done by Quellung capsular swilling and by multiplex PCR typing. Antimicrobial susceptibility was determined using Clinical and Laboratory Standards Institute recommendations.

Results: A total of 186 invasive Streptococcus pneumoniae strains were isolated. The ages of the patients ranged from 10 days to 80 years old. Prior to PCV introduction, 112 cases of invasive pneumococcal diseases (IPD) were observed and after 74 cases in overall population study. A significant reduction of the IPD incidence rate was observed in children less than 5 years old, from $1.49 / 1000$ to $0.68 / 1000$ admissions $(p=0.0005)$ compared between the two periods.

PCV-13 serotypes are decreased from $82.6 \%$ to $63.6 \%(p=0.02)$ and a reduction was also observed in penicillin resistant S. pneumoniae strains ( $43 \%$ vs. $22 \%, p=0.09)$ in the same age.

Conclusion: Data from this study suggest that PCV contributed to decrease IPD rate among children less than 5 years old. Continued surveillance is critical to measure the emerging of new serotypes and antibiotic resistance strain.

No conflict of interest 
ISPPD-0232

Controlling Pneumococcal Disease Around the Globe

\section{SEROTYPES AND GENOTYPES AMONG PNEUMOCOCCAL ISOLATES IN QATAR (2009 -2012)}

S. Doiphode ${ }^{1}$, S. Chochua², P. Hawkins², J. Klena², A. Deshmukh¹, M. Al Hajri ${ }^{3}$, L. McGee ${ }^{2}$

${ }^{1}$ Lab Medicine \& Pathology, Hamad Medical Corporation, Doha, Qatar; ${ }^{2}$ Streptococcus Laboratory, Centers for Disease Control and Prevention, Atlanta, USA ${ }^{3} \mathrm{CDC}$, Supreme Council of Health, Doha, Qatar

Background and Aims: PCV7 was introduced into Qatar in August 2005 and PCV13 in November 2010. In this study we describe serotype distribution and genotypes associated with disease in both children and adults post PCV7 and PCV13 introduction.

Methods: We characterized 242 isolates from blood $(n=153)$, cerebrospinal fluid $(n=9)$, bronchoalveolar lavage $(n=10)$, sputum $(n=50)$ and other $(n=20)$ from both children and adults in 2009-2012. All isolates were subjected to serotyping by Quellung and multilocus sequence typing (MLST).

Results: Post-PCV7 (2009-2010) included 110 isolates and post-PCV13 (2011-2012) 132 isolates were characterized. We identified 42 different serotypes with $9.5 \%(n=23)$ and $44.2 \%(n=107)$ included in PCV7 and PCV13 vaccines, respectively. PCV13 serotypes in 2009/2010 were $46.4 \%$ and in $2011 / 2012$ were $50.4 \%$ for all ages. The most prevalent serotypes were 19A (13.6\%), $3(9.9 \%), 8(6.6 \%), 1(6.6 \%)$ and $11 \mathrm{~A}(5 \%)$. Genotyping revealed diverse population of isolates with 122 sequence types (STs) identified. Seven STs represented $21.5 \%$ of isolates (ST156, ST172, ST180, ST306, ST320, ST558 and ST3377). Twenty percent of isolates belonged to one of 15 PMEN clones. Fourteen new alleles and 48 new STs were identified within this population.

Conclusion: Data from Qatar shows a low prevalence of PCV7 serotypes seven years after PCV7 introduction. Early in the PCV13 program, PCV13 serotypes still accounted for $44.2 \%$ of disease. MLST data suggests that the clonal structure is diverse, with many major PMEN clones circulating. Continued surveillance post-PCV13 introduction will assist in developing continued strategies to combat infections in children and adults in Qatar.

No conflict of interest

ISPPD-0251

Controlling Pneumococcal Disease Around the Globe

\section{STREPTOCOCCUS PNEUMONIAE CAUSING INFECTION IN CHILDREN LESS THAN 60 DAYS OF AGE: SEROTYPES AND ANTIMICROBIAL SUSCEPTIBILITY}

G. Echaniz-Aviles ${ }^{1}$, A. Soto-Noguerón ${ }^{1}$, M.N. Carnalla-Barajas ${ }^{1}$, J.C. Tinoco-Favila ${ }^{2}$, P. Arzate-Barbosa ${ }^{3}$, A. AnzuresGutiérrez ${ }^{4}$, M. Sireva-Network ${ }^{5}$

${ }^{1}$ Vaccine Evaluation, Instituto Nacional de Salud Publica, Cuernavaca Morelos, Mexico; ${ }^{2}$ Infectología, Hospital General de Durango, Durango, Mexico; ${ }^{3}$ Infectología, Instituto Nacional de Pediatria, Mexico City, Mexico; ${ }^{4}$ Infectología, Hospital General de León, León Guanajuato, Mexico

${ }^{5}$ Sireva, Network, Mexico, Mexico

Background and Aims: Streptococcus pneumoniae infections in small children are relatively unusual events, around $1-11 \%$ of neonatal sepsis, but are associated with substantial morbidity and mortalty. The aim of this study was to determine the distribution of pneumococcal serotypes and antimicrobial susceptibility of invasive and non-invasive pneumococcal diseases in infants younger than 60 days of life in 16 Mexican hospitals that participate in the SIREVA network.

Methods: An eleven year retrospective study was conducted during the period 2000-2013. Pneumococcal isolates were serotyped by the Quellung reaction with type and factor specific antisera (Statens Seruminstitut, Copenhagen, Denmark). Susceptibility testing was conducted by broth microdilution, in accordance with the Clinical and Laboratory Standards Institute (CLSI 2012) procedures for penicillin, cefotaxime, vancomycin, erythromycin, cholamphenicol and trimethoprim/sulfamethoxazole (TMP/SMX).

Results: A total of 120 pneumococcal isolates were collected from which 68\% (81/120) were from male children. Pneumonia was the most frequent diagnose $41 \%$ (49/120), followed by meningitis $26 \%$ (31/120), septicemia $19 \%$ $(23 / 120)$ and others like otitis and conjunctivitis $14 \%(17 / 120)$. The most frequent serotypes were $19 \mathrm{~F}, 23 \mathrm{~F}, 6 \mathrm{~B}$, $14,7 \mathrm{~F}$ and $35 \mathrm{~B}$. Serotype $19 \mathrm{~A}$ was isolated in $4 / 12$ meningitis and $4 / 12$ pneumonia cases. After introduction of pneumococcal conjugate vaccines in 2008, serotypes 10A, 12F, 15A, 15B and 23B were more frequently found. Except for TMP/SMX, most strains were susceptible to the antimicrobial tested.

Conclusion: Laboratory surveillance of pneumococcal serotypes causing invasive and non-invasive diseases in children less than 60 days of life is important, considering they are not protected by any vaccine.

No conflict of interest 


\title{
ISPPD-0400
}

Controlling Pneumococcal Disease Around the Globe

\section{SAFETY AND IMMUNOGENICITY OF THE 13-VALENT PNEUMOCOCCAL CONJUGATE VACCINE (PCV13) IN HIV- INFECTED ADULTS}

\author{
P. Farmaki ${ }^{1}$, N. Mangafas², M. Chini' ${ }^{2}$ N. Tsogas ${ }^{2}$, M. Theodoridou ${ }^{1}$, M. Lazanas², V. Spoulou ${ }^{1}$ \\ ${ }^{1}$ Infectious Diseases, “Aghia Sophia” Children's Hospital, Athens, Greece; ${ }^{2}$ Infectious Diseases, Red Cross General Hospital, Athens, Greece
}

\begin{abstract}
Aim and Methods: Safety and immunogenicity of PCV13 were evaluated in 25 HIV-infected adults aged 37-61 years (median 47 years) on ART with $\mathrm{VL}<40 \mathrm{c} / \mathrm{ml}$. At enrolment 12 patients were on $\mathrm{CDC} A$ (Group $\mathrm{A}$ ) and 13 on $\mathrm{CDC} B / \mathrm{C}$ (Group B), 10 had $C D 4>500$ cells/ $\mu$ l; 22 had received $\geq 1$ 23-valent pneumococcal polysaccharide vaccine (PPV23s) within the last 6 years. PCV13 was given to subjects and blood was collected at baseline and 28 day. IgG against pneumococcal serotypes (PS) 3, 19A, 14, 9V, 18 were quantified by ELISA.

Results: No serious vaccine-associated adverse events were observed during the 1- month follow up. At baseline, $>90 \%$ of patients had IgG $0.35 \mu \mathrm{g} / \mathrm{ml}$ against PS 19A, 14, 9V and $68 \%$ and $45 \%$ for PS 18 , and 3 respectively. At 1 month, geometric mean concentrations (GMC, $\mu \mathrm{g} / \mathrm{ml}$ ) for all PS increased significantly compared to baseline (PS19A $p=0.05$, PS18 $p=0.05$, PS14 $p=0.04$, PS9V $p=0.007$, PS3 $p=0.05)$. However, Group B patients achieved lower GMCs for all PS compared to Group A (1.973 vs $3.071 p=0.04,0.761$ vs $1.494 p=0.01,0.886$ vs $3.051 p=0.001$, 2.596 vs $3.787 p=0.0001$ and 0.316 vs $1.364 p=0.002$ for PS19A, PS18,PS9V, PS14 and PS3). Similarly patients with CD4<500 cells/ $\mu$ gave inferior 1 month responses to all PS, compared to those with CD4 $>500$ cells/ $\mu$ l whereas for PS3 they failed to achieve even the protective threshold.

Conclusion: PCV13 is safe and immunogenic in HIV-infected adults on ART although immune responses are affected by CD4 and stage of HIV. PS 3 is not immunogenic in subjects with CD4 $<500$ cells $/ \mu$ l.
\end{abstract}

No conflict of interest

ISPPD-0293

Controlling Pneumococcal Disease Around the Globe

\section{INVASIVE PNEUMOCOCCAL DISEASE AMONG ADULTS IN ARGENTINA (2010-2012): SEROTYPE DISTRIBUTION, ANTIBIOTIC RESISTANCE AND VACCINE SEROTYPE COVERAGE}

S. Fossati ${ }^{1}$, M. Regueira ${ }^{1}$, P. Gagetti ${ }^{2}$, D. Napoli ${ }^{1}$, A. Corso ${ }^{2}$, D. Stamboulian ${ }^{3}$, S. Pneumoniae Adult Group of Argentina $^{4}$

${ }^{1}$ Bacteriologia Clinica, INEI-ANLIS “Dr Carlos G Malbran”, Capital Federal, Argentina; ${ }^{2}$ Antimicrobianos, INEI-ANLIS “Dr Carlos G Malbran”, Capital Federal, Argentina; ${ }^{3}$ FIDEC, Fighting Infectious Diseases in Emerging Countries, Miami, USA; "Bacteriologia, INEI-ANLIS “Dr Carlos G Malbran", Capital Federal, Argentina

Background and Aims: Streptococcus pneumoniae (Spn) is a major cause of pneumonia and meningitis worldwide. We aimed to determine serotype distribution and antimicrobial susceptibility of Spn causing invasive pneumococcal disease (IPD) in adults, and serotype coverage of PCV13 and PPSV23.

Methods: Spn isolates from sterile fluids were collected in adults $>18$ years from 26 hospitals in Argentina(7 provinces and Buenos Aires City). Strains received at INEI were serotyped by Quellung reaction and MICs determined by agar dilution (CLSI-2013).

Results: Between 2010 and 2012, a total of 96 IPD patients were included (29/2010, 30/2011, 37/2012); 55 (57\%) were women and $41 / 91(45 \%)$ were $>65$ years. Strains were isolated from: blood $(74 \%)$, cerebrospinal fluid $(16.7 \%)$, peritoneal fluid (4.2\%), pleural fluid (2.1\%), other (3\%). Main diagnoses: pneumonia (69.8\%), meningitis(17.7\%), sepsis(2.1\%), pericarditis(2.1\%), other (8.3\%). More than 20 serotypes were identified: 7F (11), 1 (9), 3 (8), 5 (7), NT (7), $14(6), 19 \mathrm{~A}(6), 12 \mathrm{~F}(6), 9 \mathrm{~V}(4), 22 \mathrm{~F}(4), 8(4), 9 \mathrm{~N}(3), 11 \mathrm{~A}(2), 15 \mathrm{~B}(2), 19 \mathrm{~F}(2), 6 \mathrm{~A}(2), 6 \mathrm{C}(2), 10 \mathrm{~A}(1), 23 \mathrm{~A}(1)$, 23F (1), 33F (1), 16F (1), other (6). PCV13/PPSV23 serotype coverage was $58.3 \% / 81.2 \%$ respectively. Overall, $18.8 \%$ of isolates were penicillin non-susceptible $(15.6 \% \mathrm{MIC}=0.12-1 \mathrm{mg} / \mathrm{L} ; 3.1 \% \mathrm{MIC} \geq 2 \mathrm{mg} / \mathrm{L})$, most were serotypes $19 \mathrm{~A}$ and 6 A. Non-susceptibility rates were: $4.2 \% / 3.1 \%$ for meningitis/non-meningitis cefotaxime break-points, $2.1 \%$ amoxicillin, $6.2 \%$ meropenem, $13.5 \%$ erythromycin, $8.3 \%$ tetracycline, $31.2 \%$ trimethoprim-sulfamethoxazole. All strains were susceptible to rifampin, levofloxacin, chloramphenicol and vancomycin.

Conclusion: This is the first time that this data is presented for Argentina. The four most common serotypes were 7F, 1, 3 and 5; almost 20\% of isolates were penicillin non-susceptible. A continuous National Surveillance Program of Spn serotypes in adult IPD is warranted.

No conflict of interest 
ISPPD-0295

Controlling Pneumococcal Disease Around the Globe

\section{SEROTYPE DISTRIBUTION AND ANTIBIOTIC RESISTANCE OF STRETOCOCCUS PNEUMONIAE BEFORE AND AFTER INTRODUCTION OF 13-VALENT PNEUMOCOCCAL CONJUGATE VACCINE (PCV13) IN ARGENTINA}

S. Fossati ${ }^{1}$, P. Gagetti ${ }^{2}$, V. Reijtman ${ }^{1}$, D. Napoli ${ }^{1}$, M. Moscoloni ${ }^{1}$, L. Irazu ${ }^{3}$, M. Regueira ${ }^{1}$, S. pneumoniae Group of Argentina ${ }^{4}$, A. Corso ${ }^{2}$

${ }^{1}$ Bacteriologia Clinica, INEI-ANLIS “Dr Carlos G Malbran”, Capital Federal, Argentina; ${ }^{2}$ Antimicrobianos, INEI-ANLIS “Dr Carlos G Malbran”, Capital Federal, Argentina; ${ }^{3}$ Estadística, INEI-ANLIS “Dr Carlos G Malbran”, Capital Federal, Argentina; ${ }^{4}$ Bacteriologia, INEI-ANLIS “Dr Carlos G Malbran", Capital Federal, Argentina

Background and Aims: Diseases caused by Streptococcus pneumoniae are a major worldwide public health problem especially in children. We aimed to determine serotype distribution and antibiotic resistance of S. pneumoniae causing invasive disease(IPD) in children in $<2$ y old before and after introduction of PCV13 in the National Schedule in January 2012.

Methodology: S. pneumoniae isolates from sterile fluids (110 hospitals, 20 provinces and Buenos Aires city) from January 2010-August 2013 were serotyped by Quellung. MIC was performed by agar dilution (CLSI2013).

Results: From 1220 S. pneumoniae isolated in $<6$ old, $56 \%$ were $<2$ y old Diagnosis: pneumonia (53\%), meningitis (19.8\%), sepsis (11.8\%), other (15.4\%). We compared isolates from children < 2 y old in two periods JanuaryAugust: 2010-2011 (prePCV13, annual average $=147$ ) and 2013 (postPCV13, $n=77)$. Serotype distribution ( $n$ prePCV13/n postPCV13) was: 14 (34/7), 6B (9/2), 7F (9/5), $1(10 / 5), 3$ (7/5), 5 (16/6), 23F (7/1), 19A (11/5), 6A (8/1), $18 \mathrm{C}(6 / 0), 12 \mathrm{~F}(4 / 4), 19 \mathrm{~F}(5 / 0), 9 \mathrm{~V}(4 / 2)$, NT $(4 / 15)$, other (14/19). We observed decrease in serotype 14 (25.2\%$9.1 \%, p=0.004)$ and increase in NT $(2.4 \%-19.5 \%, p<0.0001)$ and other $(9.5 \%-27.7 \%, p=0.0008)$. The prevalence of PCV13 serotypes decreased from $86.4 \%$ in prePCV13 to $50.6 \%$ in postPCV13 $(p<0.0001)$. No significant differences in resistance were observed between prePCV13 and postCV13 periods: penicillin (PEN) MIC $\geq 0.12 \mathrm{mg} / \mathrm{L} 38 \% / 29 \%$; erythromycin (ERY) 32\%/31\%, tetracycline (TET) 19\%/32\%; cotrimoxazole (SXT) 35\%/45\%. However the number of cases of IPD associated with resistance decreased ( $n$ prePCV13/n postPCV13): PEN (56/19), ERY (47/20), TET (29/21), SXT (51/29). Resistance to chloramphenicol, levofloxacin, rifampicin nor vancomycin was not detected. Conclusion: Although PCV13 was recently introduced into the immunization schedule, we observed decrease in the number of cases of IPD, as well as in PCV13 serotypes and associated resistance. Surveillance is needed to continue monitoring the impact of PCV13-vaccination program.

No conflict of interest

ISPPD-0106

Controlling Pneumococcal Disease Around the Globe

\section{IMPACT OF RISK FROM COMORBIDITIES AND AGE ON OUTCOMES IN HOSPITALIZED PATIENTS WITH PNEUMONIA, MENINGITIS, AND SEPTICEMIA IN FRANCE}

P. Casez ${ }^{1}$, J. Fauconnier ${ }^{2}$, E.M. Gutterman ${ }^{3}$, S. Gray ${ }^{4}$, S. Munson ${ }^{4}$, N. Dartois ${ }^{5}$, J. Gaillat ${ }^{6}$

${ }^{1}$ Service d'Information et d'Evaluation Médicale, Centre Hospitalier de la Région d'Annecy, Pringy, France; ${ }^{2}$ Département d'Information Médicale, Université Joseph Fourier, Grenoble, France; ${ }^{3}$ Global Epidemiology, Via Research LLC, Princeton Junction, USA; ${ }^{4}$ Medicines Development Group, Pfizer Inc, Collegeville, USA; ${ }^{5}$ Medicines Development Group, Pfizer Inc, Paris, France; ${ }^{6}$ Service des maladies infectieuses, Centre Hospitalier de la Région d’Annecy, Pringy, France

Background and Aims: Because of the paucity of data describing the impact of risk factors on outcomes in French patients with pneumonia, meningitis, and septicemia, we quantified risk effects in population-based cohort data from the French Diagnosis Related Groups program database for residents of all ages from the Rhone-Alpes region of France who were admitted to any hospital in France in 2010.

Methods: Prespecified ICD-10 codes were used to identify cases of all-cause and pneumococcal pneumonia, meningitis, and septicemia in hospitalized patients. Distribution of pre-defined risk (high-risk [HR], at-risk [AR], no-risk [NR]) from comorbidities identified using ICD-10 codes during preceding 5 years was calculated overall and by age group. In-hospital mortality rate (IHMR) and length of stay (LOS) were calculated and compared across risk levels, overall, and by age.

Results: Proportion of each risk group varied by condition; HR was most common in septicemia patients. Risk increased with age. For all-cause pneumonia, IHMR increased with age and was highest in HR patients. Risk increased IHMR among patients with pneumococcal pneumonia overall and in adults aged $\geq 65$ years. IHMRs for HR and AR were higher than NR for all-cause meningitis and septicemia $(p<0.001)$. For pneumococcal meningitis and septicemia, IHMR was highest in HR adults aged $\geq 65$ years, although not significantly $(p>0.05)$. LOS increased with risk level. 


\begin{tabular}{|c|c|c|c|c|c|c|c|c|}
\hline \multirow[b]{2}{*}{ Disease } & \multicolumn{4}{|c|}{ All-cause } & \multicolumn{4}{|c|}{ Preumocoscal } \\
\hline & $n$ & NR & AR & NER & $n$ & NR & $A R$ & HR \\
\hline Pneumonla, \% & 25155 & 17.11 & 48.50 & 34.39 & 1044 & 34.20 & 41.09 & 24.71 \\
\hline HIMR, $\%$ & & 2.35 & $10.36^{\circ}$ & $1804^{\prime}$ & & 0.56 & $10.72^{\prime \prime}$ & $10.85^{\prime}$ \\
\hline tos, days & & 4 & 9 & 12 & & 4 & 12 & 12.5 \\
\hline Meringltis, \& & 765 & 57.12 & 23.66 & 19.22 & 78 & 39.74 & 43.59 & 16.67 \\
\hline HMR, $\%$ & & 0.69 & $9.99^{\circ}$ & $13.87^{\circ}$ & & 6.45 & 14.71 & 7.69 \\
\hline Los, days & & 4 & 13 & 14 & & 12 & 16 & 19 \\
\hline Septicemia, $\mathrm{N}$ & $\gamma 519$ & 12.96 & 30.14 & 57.91 & 215 & 25.58 & 41.40 & 33.02 \\
\hline FHMR, $\%$ & & 5.56 & $19.64^{\prime}$ & 25.06 & & 3.64 & $14,61^{\circ}$ & $23.94 \%$ \\
\hline IOS. dors & & 8 & 14 & 16 & & 6 & 14 & 11 \\
\hline
\end{tabular}

Conclusion: In France, presence of comorbidities is associated with increased IHMR and LOS for most study conditions. These data are useful for establishing vaccination policy.

Conflict of interest

ISPPD-0298

Controlling Pneumococcal Disease Around the Globe

\section{FIRST YEAR POST PCV13 VACCINE INTRODUCTION IN ARGENTINA: A HOSPITAL-BASED PNEUMONIA SURVEILLANCE IN TEN PEDIATRIC HOSPITAL UNITS}

A. Gentile ${ }^{1}$, J. Bakir ${ }^{1}$, S. Lopez Papucci ${ }^{2}$, M. Lucion ${ }^{1}$, F. Molina ${ }^{3}$, H. Abate ${ }^{4}$, A. Gajo Gane ${ }^{5}$, E. Casanueva ${ }^{6}$, V. Firpo ${ }^{7}$, A. Caruso ${ }^{8}$, A.L.D.O. Cancellara ${ }^{9}$, G. Ensinck ${ }^{2}$, N. Miguez $^{10}$, P. Scoleri ${ }^{11}$, C. Aguirre $^{12}$, N. Duci ${ }^{12}$, P. Carrizo Herrera ${ }^{13}$, M. Uranga ${ }^{14}$, N. Fernandez ${ }^{15}$

${ }^{1}$ Epidemiology, R. Gutierrez Children Hospital, Capital Federal, Argentina; ${ }^{2}$ Infectious Disease, V. Vilela Children Hospital, Rosario, Argentina;

${ }^{3}$ Pediatrics, O. Alassia Children Hospital, Santa Fe, Argentina; ${ }^{4}$ Infectious Disease, H. Notti Pediatric Hospital, Mendoza, Argentina; ${ }^{5}$ Infectious Disease, J. Pablo II Pediatric Hospital, Corrientes, Argentina; ${ }^{6}$ Infectious Disease, San Justo Children Hospital, Buenos Aires, Argentina; ${ }^{7}$ Infectious Disease, Jesus Child Hospital, Tucuman, Argentina; ${ }^{8}$ Infectious Disease, H. Quintana Children Hospital, Jujuy, Argentina; ${ }^{9}$ Infectious Disease, P. Elizalde Children Hospital, Buenos Aires, Argentina; ${ }^{10}$ Infectious Disease, Pneumococcal Working Group, Rosario, Argentina; ${ }^{11}$ Pediatrics, Pneumococcal Working Group, Santa Fe, Argentina; ${ }^{12}$ Infectious Disease, Pneumococcal Working Group, Mendoza, Argentina; ${ }^{13}$ Infectious Disease, Pneumococcal Working Group, Catamarca, Argentina; ${ }^{14}$ Infectious Disease, Pneumococcal Working Group, Buenos Aires, Argentina; ${ }^{15}$ Infectious Disease, Pneumococcal Working Group, Tucuman, Argentina

Background and Aims: In Jan/2012, PCV13 was introduced into routine immunization program, in $2+1$ schedule for children $<2$ years, in Argentina. The coverage rates were $75.7 \%$ and $43.0 \%$ for the 1 st and 2 nd dose in infants $<1$ year. Objectives: to estimate the burden and clinical-epidemiological pattern of community-acquired pneumonia (CAP) through hospital-based surveillance at pediatric sentinel units before and after PCV13 vaccine introduction in Argentina.

Methods: We compare population characteristics, annual hospitalized incidence (per 10.000 discharges) for CAP and pneumococcal-CAP (P-CAP) between pre-vaccine years: 2007-2008 (1st period)) and vaccine introduction 2012 (2nd period). Three years prospective active surveillance of CAP conducted at 10 pediatric hospital sentinel units. First year report (January-December/12).

Results: Significant reduction was observed for CAP incidence in the 10 hospitals, between both periods from 623 (5441/87331) CI95\% 607-640 to 495 (4051/82027) CI95\% 479-509 with an effectiveness of 20.73\% CI95\% 17.4423.89. In 6 hospitals, the P-CAP rates decreased $23.4 \%$, from $10.2(65 / 63441)(95 \% \mathrm{Cl} 8-13$ to 7.8$)(47 / 59890)(95 \% \mathrm{Cl}$ 6-10) without statistical significance. Out of 277 culture confirmed CAP cases of the 1st period, $196(70.8 \%)$ were P-CAP, while in the 2 nd period 59.3\% (99/167) corresponded to P-CAP ( $p=0.017)$. The percentage of children $<2$ years with P-CAP was reduced $45.4 \%(95 \% \mathrm{Cl} 14.2-65.2)$ from $44.4 \%(87 / 196)$ to $24.2 \%(24 / 99)$ for 2 nd period $(p=$ 0.001). No significant associations were found in other population characteristics between both periods.

Conclusion: In the 1st year after PCV13 introduction, there was a significant reduction in CAP incidences and in the percentage of children $<2$ years with pneumococcal-CAP.

No conflict of interest 
ISPPD-0212

Controlling Pneumococcal Disease Around the Globe

\title{
INTRODUCTION OF THE PCV13 IN ARGENTINA: ASSESSMENT OF EFFECTIVENESS TO PREVENT CONSOLIDATED PNEUMONIA IN CHILDREN
}

\author{
A. Gentile ${ }^{1}$, J. Bakir ${ }^{1}$, L. Bialorus², L. Caruso³ , M. Fernández², D. Mirra², C. Santander², M. Terluk², P. Zurdo², F. Gen- \\ tile $^{4}$ \\ ${ }^{1}$ Epidemiology, R. Gutierrez Children Hospital, Bs As, Argentina; ${ }^{2}$ Pediatrics, F. Falcon Pediatric Hospital, Bs As, Argentina; ${ }^{3}$ Pediatrics, J. Sangui- \\ netti Hospital, Bs As, Argentina; ${ }^{4}$ Diagnostic Imaging, R. Gutierrez Children Hospital, Bs As, Argentina
}

Background and Aims: In Jan/2012, PCV13 was introduced into routine immunization program, in 2+1 schedule for children <2 years, in Argentina. Pilar vaccination coverage was $85 \%$ and $94 \%$ for the 1 st and 2 nd in $<1$ year; and $61 \%$ for 3rd dose. Objective: to evaluate the effectiveness of PCV13 in Pilar Department.

Methods: Prospective population-based study after PCV13 introduction. Inclusion criteria: all children $<5$ y with clinical signs of pneumonia living in Pilar, assisted in references hospitals, between Jan/12-Dec 2013 Comparison of population characteristics, and annual consolidated pneumonia (CP) incidence (per 10.0000 discharges) between pre-vaccine years (2003-2005) and 1st two years of vaccine introduction.

Results: The study included 666 patients with suspected pneumonia; 96.4\% (642/666) had chest X-ray digital photos, CP was diagnosed in 48.1\% (309/642). Five percent of them (17/309) were laboratory-confirmed bacterial pneumonia, $52.1 \%(161 / 309)$ were female, $70.2 \%(217 / 309)<2$ years, $58.9 \%$ had underlying disease and $56.4 \%$ were vaccinated with PCV13. A significant reduction in CP incidence was observed between both periods from 750 $(204 / 27209)$ to $548(167 / 30475)$ in 2012 and 453 (138/30475) in 2013, with an effectiveness of 26.9\% (10.4-40.4) and $39.6 \%$ (25.0-51.3) respectively. Reduction for $<1$ year was $33.9 \%$ in 2012 and $44.6 \%$ in 2013 and for children $12-23$ months was $20.9 \%$ and $57.9 \%$ respectively. No significant differences were observed in the population characteristics neither in older ages between both periods.

Conclusion: Two years after PCV13 introduction into Argentina vaccination schedule, there was a significant reduction in consolidated pneumonia, mainly in $<2$ years of age.

No conflict of interest

ISPPD-0254

Controlling Pneumococcal Disease Around the Globe

\section{DISTRIBUTION OF SEROTYPES AND ANTIMICROBIAL SUSCEPTIBILITY OF STREPTOCOCCUS PNEUMONIAE IN PEDIATRIC PATIENTS WITH INVASIVE INFECTIONS}

\author{
A.C.V. Villagra' ${ }^{1}$, M.L.G. Gonzalez², J.D. Assa² \\ ${ }^{1}$ Tucuman, Hospital del Niño Jesus, San Miguel de Tucuman, Argentina; ${ }^{2}$ Tucuman, Hospital del Niño Jesus, San Miguel de Tucuman, Argentina
}

To determine frequency of Streptococcus pneumoniae vaccine serotypes in pediatric invasive infections in period of introduction of 13-valent conjugate vaccine to the national immunization. Relationship antibiotype.

Methods: A descriptive, retrospective study of 38 patients with invasive infections caused by strains of Spn, Sept 2011 to Sept 2013, Hospital del Niño Jesus. The antimicrobial susceptibility testing penicillin (Pen) and ceftriaxone (Cro) were performed by MIC using E-test bioMérieux according to Clinical and Laborartory Standards Institute (CLSI) 2012.

Results: Of 38 patients in 20 (53\%) was determined Spn serotype. 5 of them, of patients with meningitis were detected serotypes 5, 3, 7F, 23F and $18 \mathrm{C}$. The 15 patients: bacteremic pneumonia (13), 3 with pleural effusion, peritonitis (1) and occult bacteremia (1) serotypes detected: 5 (1), 4 (5), 2 (23F), 2 (14), 1 (3) and one (11A). 5 and 1 serotypes accounted for $50 \%$. The Pen and Cro MICs could not be performed in 3 strains, 34 strains were sensitive to Cro and Pen, 1 strain of meningitis had Pen MICs of $2 \mathrm{mg} / \mathrm{ml}(\mathrm{R})$. Of the 38 patients in 8 without evidence of vaccine, no vaccine 24 and 6 with insufficient doses, including here 3 patients affected by serotype 23F. We found patients affected with Spn vaccine serotypes, as 1, 5, 7F, 14 with high invasiveness. 1 and 5 are almost never nasopharyngeal colonizers therefore usually are susceptible to antibiotics. After two doses of primary vaccination between $20 \%$ and $40 \%$ of children do not achieve protective titers against serotype $23 \mathrm{~F}$, which would explain the infection in vaccinated patients

No conflict of interest 
ISPPD-0132

Controlling Pneumococcal Disease Around the Globe

\title{
PNEUMOCOCCAL SEROTYPE DISTRIBUTION PATTERNS AMONG ADULTS: GROWING EFFECTS OF PEDIATRIC PCV VACCINATION PROGRAMS
}

\author{
J.D. Grabenstein ${ }^{1}$, D.J. Weber ${ }^{2}$ \\ ${ }^{1}$ Medical Affairs \& Policy, Merck Vaccines, West Point, USA; ${ }^{2}$ Department of Medicine, University of North Carolina, Chapel Hill, USA
}

Background \& Aims: Indirect effects of widespread pediatric use of pneumococcal conjugate vaccines (PCVs) influence the frequency of serotype-specific pneumococcal disease among adults.

Methods: To characterize the effect of PCVs on adult pneumococcal serotype distribution, we systematically reviewed the literature. From all eligible papers, differences in serotype distribution for invasive pneumococcal disease (IPD) isolates matching the 23-valent pneumococcal polysaccharide vaccine (PPSV23) or PCV13 were assessed. Similarly, differences in IPD rates corresponding to PPSV23 and PCV13 serotypes were evaluated. Reports were stratified by pre- and post-introduction of childhood PCV programs.

Results: For IPD serotype distribution among adults in 21 reports before widespread pediatric use of PCV, the median differential between the 23 and 13 vaccine serotypes was 16\% (interquartile range, IQR, 12-22). For 8 studies after widespread pediatric PCV7 use, the median differential was $25 \%$ (IQR 19-29) ( $p<0.0002$, compared to $16 \%)$. For serotype-specific IPD incidence rates among adults in 10 reports before widespread pediatric PCV7 use, the median 23 -vs-13 differential was 0.5 cases per 100,000 population (IQR 0.3-4.9). For 5 studies after widespread pediatric PCV use, the median differential was 4.6 cases per 100,000 (IQR 2.8-5.7) ( $p<0.01$, compared to 0.5). Corresponding values for settings with widespread pediatric PCV13 programs will be presented on site. Conclusion: The differential between the serotypes included in PPSV23 and PCV13 has widened, both as proportions of IPD isolates and clinical incidence rates. These differentials are relevant to vaccination strategies and vaccine formulation decision-making. These differences may widen further, with more extensive pediatric uptake of PCV10 or PCV13.

Conflict of interest

ISPPD-0211

Controlling Pneumococcal Disease Around the Globe

\section{3-VALENT POLYSACCHARIDE PNEUMOCOCCAL VACCINES INDUCE ROBUST ANTIBODY RESPONSES AGAINST LETHAL PNEUMOCOCCAL SEROTYPES}

K.L. Ciprero ${ }^{1}$, R.D. Marchese ${ }^{1}$, P. Richard ${ }^{2}$, M. Baudin², L.K. Musey ${ }^{3}$, S.B. Manoff ${ }^{3}$, D. Radley ${ }^{3}$, J. Stek ${ }^{3}$, B. Soubeyrand ${ }^{4}$, J.D. Grabenstein ${ }^{5}$, S.I. Samson ${ }^{6}$

${ }^{1}$ Clinical, MSD, West Point, USA; ${ }^{2}$ Clinical, SPMSD, Lyon, France; ${ }^{3}$ Clinical, MSD, West Point, USA; ${ }^{4}$ Medical, SPMSD, Lyon, France; ${ }^{5}$ Medical, MSD, West Point, USA; ${ }^{6}$ Medical, SPMSD, Lyon, France

Background and Aims: PNEUMOVAX ${ }^{\circledR}$ II (also called PNEUMOVAX ${ }^{\circledR} 23$ ), a 23-valent polysaccharide pneumococcal vaccine (PPV23), covers $65 \%$ to $91 \%$ of the isolates responsible for invasive pneumococcal disease. Harboe and colleagues (PLOS Med 2009) showed 3.3- to 5.7-fold increased case-fatality rates associated with pneumococcal disease caused by serotypes $31,11 \mathrm{~A}, 35 \mathrm{~F}, 17 \mathrm{~F}, 3,16 \mathrm{~F}, 19 \mathrm{~F}, 15 \mathrm{~B}$, and $10 \mathrm{~A}$ in comparison to serotype 1 as control. This study evaluated the immune response of PNEUMOVAX ${ }^{\circledR}$ II for four of these nine serotypes (10A, 11A, 15B, and 17F), that are included in PNEUMOVAX ${ }^{\circledR}$ II but not in pneumococcal conjugate vaccines.

Methods: Serotype-specific IgG geometric mean concentrations (GMCs) for these four serotypes were measured by ELISA in 104 subjects $>50$ years old (mean 58, range 52-72) who were enrolled in a study evaluating the safety and immunogenicity of one dose of PNEUMOVAX ${ }^{\circledR} \|$.

Results: For serotypes 10A, 11A, 15B and 17F, 1 month post-vaccination, GMCs were 6.5, 4.3, 14.7, and $5.1 \mathrm{mcg} /$ $\mathrm{mL}$, respectively. GMC fold rises from baseline were $9.0,4.5,8.4$, and 11.5 , respectively. The percentage of subjects achieving a $>2$-fold increase of antibody concentration post-vaccination was $90 \%, 85 \%, 88 \%$ and $89 \%$, respectively. Conclusion: PNEUMOVAX ${ }^{\circledR}$ II induces a robust immune response in adults to pneumococcal serotypes 10A, 11A, $15 \mathrm{~B}, 17 \mathrm{~F}$, which were associated with elevated case-fatality rates. It has is the only pneumococcal vaccine that includes these

Conflict of interest

ISPPD-0133

Controlling Pneumococcal Disease Around the Globe

\section{DIFFERENCES IN SERIOUS CLINICAL OUTCOMES OF INDIVIDUAL PNEUMOCOCCAL SEROTYPES AMONG ADULTS}

\author{
J.D. Grabenstein ${ }^{1}$, L.K. Musey ${ }^{2}$ \\ ${ }^{1}$ Medical Affairs \& Policy, Merck Vaccines, West Point, USA; ${ }^{2}$ Clinical Research, Merck Research Laboratories, Upper Gwynedd, USA
}

Background and Aims: Streptococcus pneumoniae serotypes differ in their clinical manifestations among adults, varying in propensity for disease severity, invasiveness, and lethality. To characterize differentials in serious outcomes by various pneumococcal serotypes, we systematically reviewed the literature.

Methods: After distilling 658 hits to 27 relevant articles, statistically significant differences in serotypes associated 
with empyema, meningitis, septic shock, necrotizing pneumonia, and case-fatality rates were assessed. Serotypes repeatedly associated with elevated risk of serious clinical outcomes were evaluated in terms of serotypes included in licensed adult pneumococcal vaccines (i.e., 23-valent pneumococcal polysaccharide vaccine (PPSV23) and 13-valent pneumococcal conjugate vaccine (PCV13)).

Results: Among adult studies evaluating serious clinical outcomes, the following serotypes were repeatedly associated with elevated risk:

\begin{tabular}{|l|l|l|}
\hline Manifestation & Studies & Serotypes with Elevated Risk \\
\hline Empyema & 4 & $1,3,5,7 \mathrm{~F}, 8,19 \mathrm{~A}$ \\
\hline Meningitis & 7 & Repeatedly, 16B, 10A, 12F, 15B, 19F, 23F \\
\hline Septic shock & 3 & $3,19 \mathrm{~A}$ \\
\hline Necrotizing pneumonia & 1 & 3 \\
\hline Case-fatality & 18 & $3,6 \mathrm{~B}, 9 \mathrm{~N}, 11 \mathrm{~A}, 16 \mathrm{~F}, 19 \mathrm{~F}, 19 \mathrm{~A}$ \\
\hline
\end{tabular}

Conclusion: Pneumococcal serotypes most often associated with a significant elevation in risk for serious clinical outcomes were included in both PPSV23 and PCV13. Four studies found elevated risk from serotype 6A (unique to PCV13). Thirteen studies found notably elevated risk from serotypes unique to PPSV23 (case-fatality: 11A and $9 \mathrm{~N}$, meningitis: 15B, 10A $12 \mathrm{~F}$ ). Seven studies found elevated risk from serotypes not represented in any vaccine formulation. The pneumococcal serotypes repeatedly associated with elevated risk of serious outcomes in adults are an important consideration for vaccine policy making.

Conflict of interest

ISPPD-0149

Controlling Pneumococcal Disease Around the Globe

\title{
NATIONWIDE SEROTYPE DISTRIBUTION OF INVASIVE PNEUMOCOCCAL ISOLATES IN CHILDREN AND ADULTS IN THAILAND, 2008-2012
}

\author{
C.J. Gregory ${ }^{1}$, A. Kerdsin², W. Paveenkittiporn ${ }^{2}$, S. Bhengsri ${ }^{1}$, H.C. Baggett ${ }^{1}$, S. Dejsirilert ${ }^{2}$, S. Sirinavin ${ }^{3}$
}

${ }^{1}$ Global Disease Detection Center, Thailand MOPH-US CDC Collaboration, Nonthaburi, Thailand; ${ }^{2}$ National Institute of Health, Department of Medical Sciences Thailand Ministry of Public Health, Nonthaburi, Thailand; ${ }^{3}$ Department of Pediatrics, Faculty of Medicine Ramathibodi Hospital Mahidol University, Nonthaburi, Thailand

Background: Pneumococcal conjugate vaccine (PCV) is not currently in Thailand's national vaccination schedule, and pneumococcal serotype data from Thailand have been reported only for a limited number of isolates and geographic areas. We evaluated serotype distribution of IPD isolates from multiyear nationwide surveillance and vaccine serotype coverage in individuals $<5$ years and $\geq 5$ years old.

Methods: Pneumococcal isolates from 47 hospitals in Thailand's nationwide Invasive Bacterial Infection Surveillance (Thai-IBIS) network from 2008-2012 were sent to the Thailand National Institute of Health for confirmation and serotyping by sequential multiplex PCR. The 6 geographic regions in Thailand contributed 84 to 251 isolates each. Results: Among 836 IPD isolates, 97 were in children $<5$ and 739 in persons $\geq 5$ years old. Of these, 785 were from blood, 35 from cerebrospinal fluid (CSF) and $90.3 \%$ could be serotyped. Predominant serotypes in children $<5$ were $6 \mathrm{~B}(26.4 \%), 14(22.0 \%)$ and $19 \mathrm{~F}(9.9 \%)$. In persons $\geq 5$ years, most common were serotypes $3(10.7 \%), 6 \mathrm{~B}(9.7 \%), 19 \mathrm{~A}$ $(8.7 \%)$ and $23 \mathrm{~F}(8.7 \%)$. Among children $<5,74 \%$ and $82 \%$ of serotyped isolates were contained in 10 -valent PCV (PCV10) and PCV13, respectively, compared to $48 \%$ and $70 \%$ in $\geq 5$ year olds.

Conclusion: Based on the largest reported sample of isolates in Thailand, PCV10 and PCV13 would cover $>70 \%$ of IPD in children $<5$ years, and PCV13 would cover the majority of IPD in older individuals. Vaccine serotype coverage for young children is close to previous estimates, but the large number of isolates from older individuals and national scope of the sample contribute important data for vaccine policy considerations.

No conflict of interest

ISPPD-0185

Controlling Pneumococcal Disease Around the Globe

\section{ACUTE OTITIS MEDIA AND PNEUMONIA IN YOUNG CHILDREN IN ICELAND: AN EARLY REDUCTION OF INCIDENCE AFTER PCV-10 IMMUNIZATION}

\author{
S. Sigursson ${ }^{1}$, K.G. Kristinsson², H. Erlendsdóttir², B. Hrafnkelsson ${ }^{3}$, A. Haraldsson ${ }^{4}$
}

${ }^{1}$ Faculty of Medicine, University of Iceland, Reykjavík, Iceland; ${ }^{2}$ Department of Clinical Microbiology, Landspitali - University Hospital Iceland \& University of Iceland, Reykjavík, Iceland; ${ }^{3}$ Department of Mathematics, University of Iceland, Reykjavík, Iceland; ${ }^{4}$ Children's Hospital Iceland, Landspitali - University Hospital Iceland \& University of Iceland, Reykjavík, Iceland

Background: Streptococcus pneumoniae is a common pathogen of pneumonia and acute otitis media (AOM) in children. PCV-10 was introduced to the childhood immunization program in Iceland in 2011. The aim of the study was to evaluate the effect on hospital visits and admissions in a population not earlier vaccinated against pneumococci.

Methods: The number of children $<15$ months of age visiting or admitted to The Children's Hospital for pneumonia 
or AOM from January 2008 through March 2013 were studied retrospectively. Children <15 months of age of each yearly birth cohort were included. Children born in 2011 (vaccinated) were compared to children born in 2008-2010 (unvaccinated) (odds ratio and likelihood ratio test).

Results: The mean annual incidence/1000 children $<15$ months of age with AOM was 106 (standard deviation (SD):15.4) and for children born in 2011 the incidence was 85, significantly lower than for children born in the previous 3 years $($ mean $=113 ; \mathrm{SD}: 7.2$; odds ratio $(\mathrm{OR}): 0,720 ; 95 \% \mathrm{Cl}: 0,632-0,820, p=10-6)$. For pneumonia the mean annual incidence was 28 (SD:5.7), and for 2011 it was 20, significantly lower in the vaccinated group (mean = 31; SD:2.8; OR:0,652; $95 \% \mathrm{Cl}: 0,508-0,837, \mathrm{p}<0.006)$. For comparison, the incidence of acute bronchiolitis in $2008-$ 2011 was 30, 34, 52, and 47 respectively.

Conclusion: A significant reduction in hospital visits and admissions due to AOM and pneumonia was seen in vaccinated children $<15$ months of age very soon after initiation of the immunisation with PCV-10. The early effect of the immunization in this young age group is encouraging.

Conflict of interest

ISPPD-0393

Controlling Pneumococcal Disease Around the Globe

\begin{abstract}
FIVE YEARS OF POST-LICENSURE EXPERIENCE WITH THE 10-VALENT PNEUMOCOCCAL NON-TYPEABLE HAEMOPHILUS INFLUENZAE PROTEIN D CONJUGATE VACCINE (PHID-CV): A REVIEW
\end{abstract}

W.P. Hausdorff ${ }^{1}$, T. Mrkvan ${ }^{1}$, M. Moreira ${ }^{1}$, J. Ruiz Guiñazú ${ }^{1}$, D. Borys ${ }^{1}$

${ }^{1}$ GlaxoSmithKline Vaccines, Wavre, Belgium

Background and Aims: Both extended valency pneumococcal conjugate vaccines (PCVs) in use today were originally licensed based on immunogenicity comparison with the heptavalent formulation. To understand how this has translated into public health impact, we reviewed the considerable amount of efficacy and effectiveness data generated with GlaxoSmithKline Vaccines' 10-valent PCV, PHiD-CV, since its licensure in 2008-2009.

Methods: PHiD-CV efficacy and effectiveness data for invasive pneumococcal disease (IPD) and pneumonia from 2 double-blind randomized controlled trials (DBRCTs), FinIP and COMPAS (focusing on intent-to-treat analyses), and multiple post-marketing surveillance (PMS) studies were reviewed.

Results: The DBRCTs and PMS studies demonstrated robust efficacy and effectiveness of infant and childhood immunization with PHiD-CV against IPD and pneumonia across a wide range of epidemiological, socio-economic and geographical settings (Table). In addition, decreases in IPD due to cross-reactive serotypes in the vaccineeligible age groups were evident from PMS studies in several countries. Significant reductions in vaccine-type IPD or nasopharyngeal carriage were also apparent in some vaccine-ineligible age groups in Finland and Brazil, suggestive of herd effects.

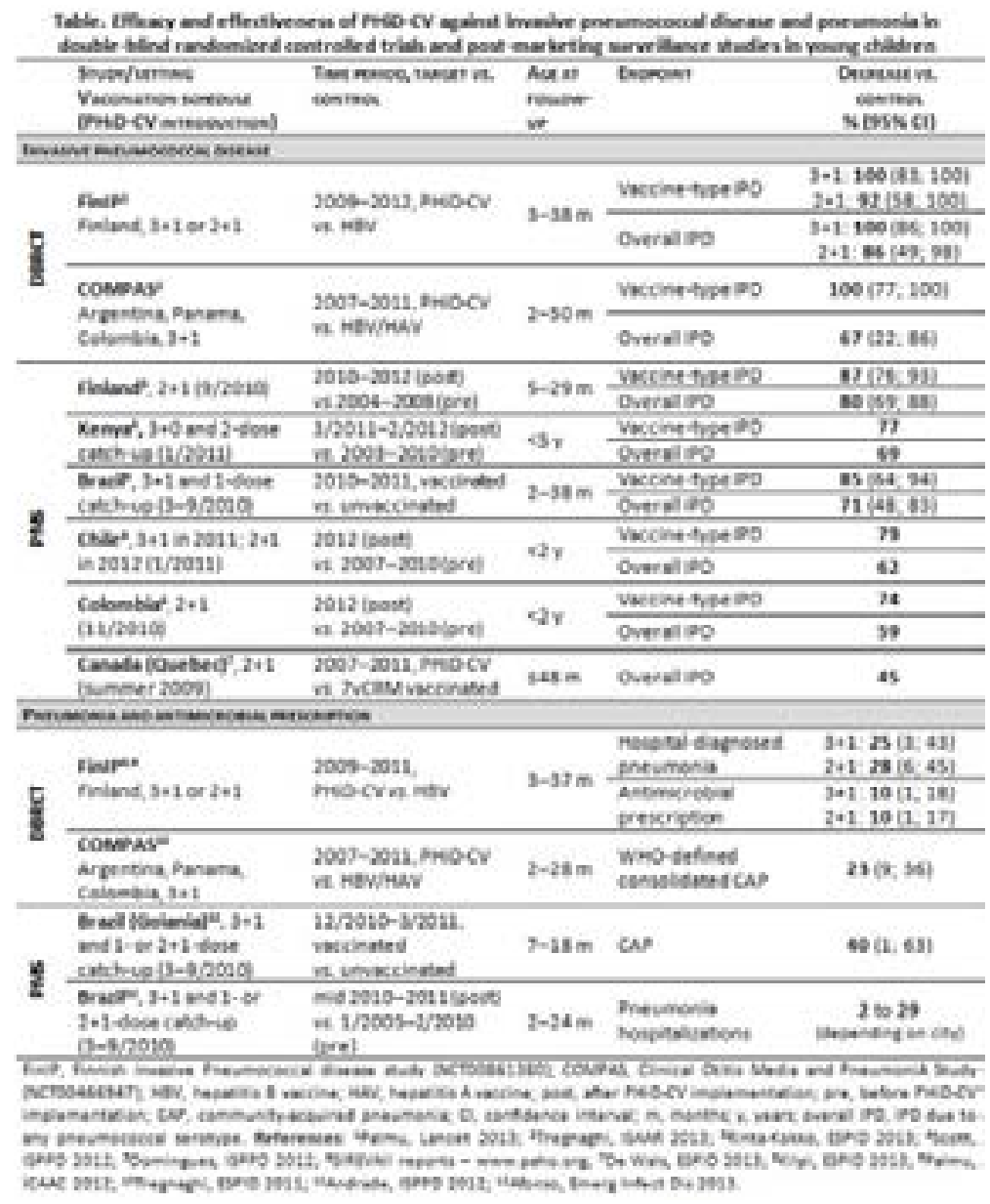


Conclusion: Immunization of infants and young children with PHiD-CV in different schedules, settings and populations have led to considerable reductions in vaccine-type and overall IPD, with early indications of herd effects and possible cross-protection against 6A and 19A IPD. PHiD-CV also proved effective in preventing pneumonia and reducing antibiotic consumption. These results highlight the public health value of PHiD-CV. Funding: GlaxoSmithKline Biologicals SA

Conflict of interest

ISPPD-0108

Controlling Pneumococcal Disease Around the Globe

\title{
THE BURDEN AND REPORTABLE CAUSATIVE AGENTS ASSOCIATED WITH HOSPITALIZED PNEUMONIA, MENINGITIS, AND SEPTICEMIA IN NORWAY (2008-2009)
}

\author{
S. Munson ${ }^{1}$, D. Lambrelli ${ }^{2}$, M. Raluy ${ }^{2}$, D. Eriksson ${ }^{2}$, R. Wasiak ${ }^{2}$, R. Hollingsworth ${ }^{1}$, R. Isturiz ${ }^{1}$, S. Gray $^{1}$ \\ ${ }^{1}$ Medicines Development Group, Pfizer, Collegeville, USA; ${ }^{2}$ Health Economics and Epidemiology, Evidera, London, United Kingdom
}

Background and Aims: Estimate the burden and infectious agents associated with pneumonia, meningitis, and septicemia in Norway from 2008-2009.

Methods: This retrospective database study examined the causative agents, hospitalization rates, in-hospital mortality rates (IHMR), and length of stay (LOS) of patients with pneumonia, meningitis, and septicemia identified through reported infectious agents and ICD-10 codes. Data from patients matched through a unique patient identifier between the Norwegian Patient Registry (NPR; hospitalization data) and Surveillance System for Communicable Disease (MSIS; laboratory-confirmed cases of 58 notifiable infectious diseases and 14 pathogens) was used.

Results: Across the two study years, 793, 125, and 335 cases of pneumonia, meningitis, and septicemia, respectively, were matched using NPR and MSIS. Of the pathogens reportable to MSIS, Streptococcus pneumoniae was associated with approximately $90 \%$ of hospitalizations and deaths due to pneumonia; Mean LOS was 10.6 days. IHMR was $5.8 \%$. S. pneumoniae was also the most common pathogen associated with meningitis (62\%), followed by Neisseria meningitidis serogroup B (15\%). S. pneumoniae caused all meningitis deaths; IHMR was 9.1\%. Mean LOS was 16.6 days. For septicemia, the most frequent infectious agents were S. pneumoniae (35\%), Staphylococcus pyogenes $(27 \%)$, and Streptococcus agalactiae (24\%). Overall IHMR associated with these pathogens ranged from 8.5\%-21.6\%; mean LOS ranged from 14.2-16.4 days. For all conditions, incidence rates were highest in adults aged $\geq 65$ years and children aged $0-4$ years; IHMR generally increased with age.

Conclusion: Of reportable pathogens, S. pneumoniae was the leading infectious agent in Norway across all study years, diseases, and age groups.

Conflict of interest

ISPPD-0107

Controlling Pneumococcal Disease Around the Globe

\section{THE BURDEN OF HOSPITALIZED ALL-CAUSE AND PNEUMOCOCCAL MENINGITIS AND SEPTICEMIA IN SPAIN (2010)}

S. Munson ${ }^{1}$, S. Gray ${ }^{1}$, M. Raluy², D. Lambrelli², R. Wasiak ${ }^{2}$, R. Hollingsworth ${ }^{1}$, R. Isturiz ${ }^{1}$, A. Gil ${ }^{3}$

${ }^{1}$ Medicines Development Group, Pfizer, Collegeville, USA; ${ }^{2}$ Health Economics and Epidemiology, Evidera, London, United Kingdom;

${ }^{3}$ Department of Preventive Medicine \& Public Health \& Medical Immunology \& Microbiology, Rey Juan Carlos University, Madrid, Spain

Background and Aims: Estimate the incidence of hospitalized all-cause and pneumococcal meningitis (acute cerebrospinal meningitis (ACM) and pneumococcal meningitis (PM) and septicemia (ACS and PS) among all ages in Spain in 2010.

Methods: This retrospective study utilized IASIST, a Spanish database covering $>65 \%$ of National Health System discharges, to determine hospitalization rates, in-hospital mortality rates, co-morbidities, and length of stay (LOS) of hospitalized residents with an ICD-9 diagnosis code of all-cause or pneumococcal meningitis or septicemia. Population data were provided by IASIST $(10,984,724)$.

Results: 1093 and 9835 hospitalized patients were diagnosed with ACM or ACS, respectively; $11.4 \%$ and $1.9 \%$ of these patients had ACM or ACS associated with Streptococcus pneumoniae. The incidence of hospitalized ACM was 10.2/100,000; the incidence of ACS was 91.4/100,000. For ACM and PM, incidence rates were highest in children aged $<4$ years $(42.5 / 100,000 ; 3.7 / 100,000$, respectively), followed by adults aged $\geq 65$ years $(11.9 / 100,000$; $2.5 / 100,000)$. Incidence of ACS and PS was highest in adults aged $\geq 65$ years $(316.5 / 100,000 ; 5.2 / 100,000)$, and children aged $<4$ years $(217.0 / 100,000 ; 1.5 / 100,000)$. In-hospital mortality rates for ACM and ACS generally increased with age, averaging $4.3 \%$ and $29.7 \%$, respectively, for all age groups combined. LOS was similar for ACM and ACS, averaging 11.3 and 13.9 days, respectively. The most frequent co-morbidities for ACM and ACS were central nervous system disorders and renal disease, respectively; co-morbidities increased with age.

Conclusion: Meningitis and septicemia remain significant sources of morbidity and mortality in Spain in the youngest and oldest age groups.

Conflict of interest 


\title{
RESERVOIR AND TRANSMISSION DYNAMICS OF MULTISTRAIN BACTERIAL PATHOGENS
}

\author{
I. Megiddo ${ }^{1}$, E.Y. Klein², R. Laxminarayan ${ }^{3}$, D.L. Smith ${ }^{4}$
}

${ }^{1}$ Center for Disease Dynamics Economics \& Policy, Center for Disease Dynamics Economics \& Policy, Washington, USA; ${ }^{2}$ Center for Advanced Modeling Department of Emergency Medicine, Johns Hopkins University, Boltimore, USA; ${ }^{3}$ Center for Disease Dynamics Economics \& Policy, Center for Disease Dynamics Economics \& Policy, New Delhi, India; ${ }^{4}$ Johns Hopkins Bloomberg School of Public Health, Johns Hopkins University, Washington, USA

Background and Aims: Flexible and extensible complex mathematical models provide an in silico laboratory for testing ideas about the factors that scale from individuals to populations and affect policy. Here we present a model of transmission and resistance applicable to Streptococcus pneumoniae and other clinically important bacteria that commonly colonize hosts and only occasionally cause disease. For these opportunistic pathogens, the colonizing bacteria (but usually not the bacteria in an infection) constitute the reservoir of contagion. Even as medicine focuses on managing invasive disease, public health and policy must also consider the reservoir dynamics that drive vaccineinduced strain replacement and the evolution of resistance to antibiotics.

Methods: Our new Markov-chain model shows the reservoir and transmission dynamics of colonizing bacteria simultaneously considers several biological phenomena.

Results and Conclusion: The effects of these biological factors on policy can lead to misleading conclusions if the wrong assumptions are made. We predict the effects of the factors in a flexible framework and identify the following to be important for bacterial reservoir dynamics and thus for policy: differences in the transmissibility, invasiveness, immunogenicity, and persistence of strains; strain-specific, cross-, or strain-transcending immunity; simultaneous colonization with multiple strains with no strain interaction, and with direct inhibition and competitive dominance; bacterial population responses to drug treatment and the evolution of drug resistance; and changes in immunity that are related to the host's age.

No conflict of interest

ISPPD-0429

Controlling Pneumococcal Disease Around the Globe

\section{EARLY IMPACT OF 3+0 SCHEDULE OF 13VPCV IN AUSTRALIAN CHILDREN AND ADULTS}

\author{
S. Jayasinghe ${ }^{1}$, C. Chiu $^{1}$, R. Menzies ${ }^{1}$, C. Bareja ${ }^{2}$, V. Krause ${ }^{3}$, P. Mclntyre ${ }^{1}$
}

${ }^{1}$ National Centre for Immunisation Research and Surveillance, The Children's Hospital at Westmead, Westmead, Australia; ${ }^{2}$ Vaccine Preventable Diseases Surveillance Section Office of Health Protection, Department of Health, Canberra, Australia; ${ }^{3}$ Centre for Disease Control, Department of Health Northern Territory Government, Darwin, Australia

Background \& Aims: On 1st July 2011, PCV13 replaced PCV7 in the childhood pneumococcal vaccination program in Australia. The unique schedule of 3 primary doses with no booster $(3+0)$ was continued for $13 \mathrm{vPCV}$. A single $13 \mathrm{vPCV}$ dose was offered, for one year, to children aged 12-35 months who had completed a 7vPCV course. This study compares impact of 13vPCV program in the first year with that in the same period post-7vPCV introduction.

Methods: IPD notifications to the national passive surveillance system, NNDSS, were used in the study. Age and serotype specific incidence rate ratios (IRRs) of IPD due to 13-non-7v types were calculated for pre- (2009-10) vs. the first complete calendar year (2012) post-13vPCV introduction. The IRRs for 13v-non-7v were compared with those for $7 \mathrm{v}$ types in comparable pre- versus post-7vPCV periods.

Results:

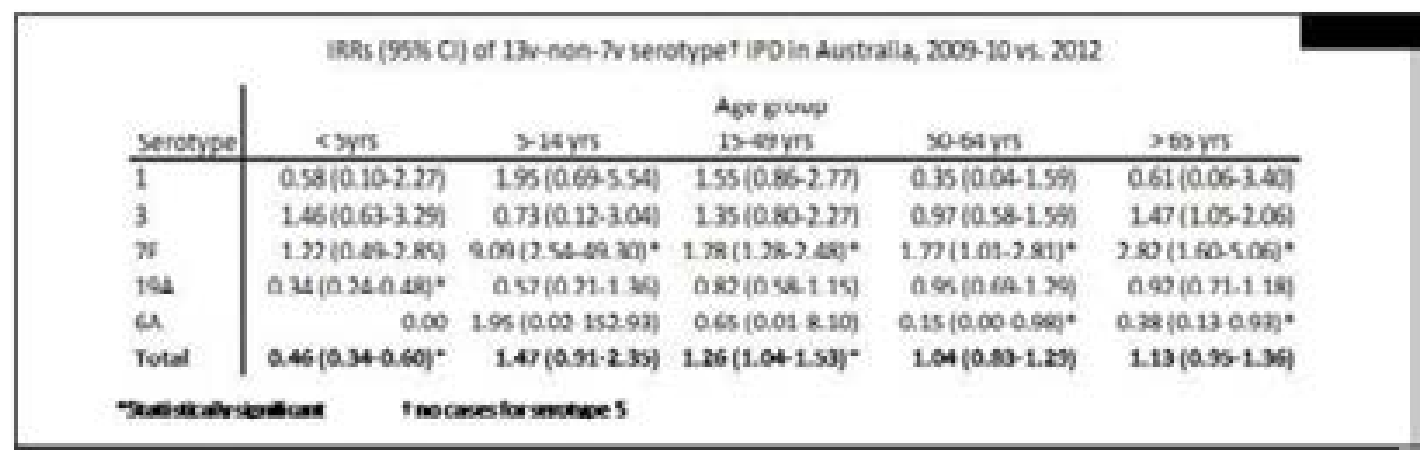

In children $<5$ y of age, 66 isolates of $13 v-n o n-7 v$ IPD were identified in 2012 compared with the average for 2009-10 of 140.5, a significant reduction in total $13 v-$ non-7v (54\%) and in 19A (66\%). There were non-significant decreases in 19A in those $>5$ years, offset by increases in 7F. In the comparable period post-7vPCV introduction in 2005 , vaccine type IPD decreased $79 \%$ and $27-36 \%$ in $<5$ and $>5$ year ages respectively.

Conclusion: A marked reduction in 13v-non-7v IPD was seen post-13vPCV introduction in the target age group and all young children. The impact of 13vPCV on IPD due to these 6 serotypes was less compared to that from 7vPCV on vaccine type IPD in a comparable period.

No conflict of interest 
ISPPD-0319

Controlling Pneumococcal Disease Around the Globe

\title{
ACUTE MENINGOENCEPHALITIS WITH STREPTOCOCCUS PNEUMONIAE IN CHILD - CASE REPORT
}

\author{
G. Jugulete ${ }^{1}$, M. Luminos ${ }^{1}$, M. Merisescu ${ }^{1}$, E. Osman ${ }^{1}$, O. Dorobat ${ }^{1}$
}

${ }^{1}$ Pediatric, Institute of Infectious Diseases “Prof. dr. Matei Bals”, Bucharest, Romania

Introduction: Streptococcus pneumoniae is a leading cause of serious diseases among children worldwide. The development and increasing prevalence of multiple-antibiotic-resistant strains has made prevention of pneumococcal disease a priority. The mortality rate of untreated bacterial meningitis approaches $100 \%$ and, even with optimal therapy, morbidity and mortality can occur sometime.

Methods: We present the case of 5-month-old infants, unvaccinated with pneumococcal vaccine, admitted to the National Institute of Infectious Diseases 'Prof. Dr. Matei Bals', diagnosed with acute meningoencephalitis with $S$. pneumoniae, serotype 14 . The child was transferred to our clinic from another Pediatric Clinic, in critical condition after cardiopulmonary arrest resuscitation. Positive diagnosis was established on clinical criteria, laboratory and imaging data.

Results: Evolution was unfavorable, with clinical, hemodynamics and neurological deterioration, requiring ventilatory support since the first day of hospitalization, subsequent to septic shock, multiple organ failure, coma and death, despite proper treatment instituted.

Conclusion:

1. Severe pneumococcal sepsis and meningoencephalitis with fatal outcome to an infant previously hospitalized for a viral bronchiolitis, without other risk factors associated.

2. Fatale invasive pneumococcal disease to an unvaccinated infant with pneumococcal conjugate vaccine, despite appropriate antibiotic therapy and intensive care treatment.

No conflict of interest

ISPPD-0161

Controlling Pneumococcal Disease Around the Globe

\section{REAL-TIME SURVEILLANCE TO EVALUATE THE IMPACT OF THE PNEUMOCOCCAL CONJUGATE VACCINE (PCV) IN ENGLAND AND WALES}

\author{
P. Kaye ${ }^{1}$, R. Malkani ${ }^{1}$, M. Slack ${ }^{2}$, S. Ladhani ${ }^{1}$, R. Borrow ${ }^{3}$, N. Andrews ${ }^{4}$, E. Miller ${ }^{1}$ \\ ${ }^{1}$ Immunisation Hepatitis and Blood Safety Dept, Public Health England, London, United Kingdom; ${ }^{2}$ Respiratory and Vaccine Preventable Bacte- \\ ria Reference Unit, Public Health England, London, United Kingdom; ${ }^{3}$ Public Health Laboratory Manchester Medical Microbiology Partnership, \\ Public Health England, Manchester, United Kingdom; ${ }^{4}$ Statistics Unit, Public Health England, London, United Kingdom
}

Background and Aims: PCV7 was introduced in England and Wales (E\&W) in September 2006 and replaced by PCV13 from April 2010. To provide rapid evaluation of the impact of PCV, the Health Protection Agency (now PHE) established a national real-time enhanced IPD surveillance system.

Methods: Weekly computerised reports of Invasive Pneumococcal Disease (IPD) cases from all laboratories in E\&W are reconciled with weekly data from pneumococcal isolates sent to the reference laboratory for serotyping creating one data set. Children in PCV-eligible cohorts are followed up each week for vaccination history risk factors for IPD and outcome by telephone and postal questionnaire. Vaccine failures are rapidly identified and serotype-specific IgG antibody testing offered to confirm adequate responses to subsequent PCV doses. Bi-weekly counts of IPD cases by age and serotype are collated into cumulative graphs and published on the internet.

Results: 3139 cases of IPD in vaccine-eligible children were followed up by October 2013. Vaccine histories were obtained for $99.9 \%$, identifying 77 PCV7 and 73 PCV13 vaccine failures. 187 children died of which $75 \%$ were attributable to IPD. Information on clinical risk factors was obtained for over $90 \%$. The first efficacy estimates for the new serotypes in PCV13 (78\%) were available within a year and the cumulative graphs showed rapid generation of herd protection in older age groups.

Conclusion: The enhanced IPD surveillance system in E\&W allows rapid monitoring of the impact of PCV vaccination, including evaluation of herd protection, serotype replacement and the serotype-specific protection afforded to the vaccinated child.

No conflict of interest 
ISPPD-0466

Controlling Pneumococcal Disease Around the Globe

\section{IMPACT OF 13-VALENT PNEUMOCOCCAL CONJUGATE VACCINE (PCV13) ON INVASIVE PNEUMOCOCCAL DISEASE (IPD) AMONG ADULTS IN THE U.S.}

L. Kim ${ }^{1}$, T. Taylor ${ }^{1}$, T. Pondo ${ }^{1}$, E. Zell ${ }^{2}$, M. Farley ${ }^{3}$, W. Schaffner ${ }^{4}$, A. Thomas ${ }^{5}$, A. Reingold ${ }^{6}$, L. Harrison ${ }^{7}$, R. Lynfield ${ }^{8}$, C. Lexau ${ }^{8}$, S. Zansky ${ }^{9}$, S. Petit ${ }^{10}$, M. Barnes ${ }^{11}$, J. Bareta $^{12}$, C. Whitney ${ }^{1}$

${ }^{1}$ Respiratory Diseases Branch, Centers for Disease Control and Prevention, Atlanta, USA; ${ }^{2}$, Stat-Epi Associates Incorporated, Atlanta, USA ${ }^{3}$ Department of Medicine, Emory University and Atlanta VA Medical Center, Atlanta, USA; ${ }^{4}$ Department of Medicine, Vanderbilt University, Nashville, USA; ${ }^{5}$ Acute and Communicable Disease Prevention Program, Oregon Public Health Division, Portland, USA; ${ }^{6}$ Division of Epidemiology, University of California Berkeley, Berkeley, USA; ${ }^{7}$ Department of International Health, Johns Hopkins Bloomberg School of Public Health, Baltimore, USA; ${ }^{8}$ Infectious Diseases Division, Minnesota Department of Health, St. Paul, USA; ${ }^{9}$ Bureau of Communicable Disease Control, New York State Department of Health, Albany, USA; ${ }^{10}$ Epidemiology and Emerging Infections Program, Connecticut Department of Public Health, Hartford, USA; ${ }^{11}$ Emerging Infections Program, Colorado Department of Public Health and Environment, Denver, USA; ${ }^{12}$ Infectious Disease Epidemiology Bureau, New Mexico Department of Public Health, Santa Fe, USA

Background and Aims: In February 2010, PCV13 replaced 7-valent pneumococcal conjugate (PCV7) vaccine in the U.S. infant immunization schedule with catch-up for children $<5$ years. We evaluated the impact of PCV13 on adult disease.

Methods: IPD cases (isolation of pneumococcus from sterile sites) among adults $\geq 18$ years were identified through Active Bacterial Core surveillance (ABCs) during July 2004-June 2012. Isolates were serotyped by Quellung and classified as PCV5 (19A, 7F, 3, 5, 1) and non-PCV13 serotypes. We used pre-PCV13 observed cases in time-series models to forecast post-PCV13 incidence in the absence of PCV13 (counterfactual scenario). PCV13 impact was the difference between forecasted incidence and observed incidence. We derived incidence estimates and confidence intervals from the distribution of Monte-Carlo simulations.

Results: ABCs identified 26,061 adult IPD cases. Average IPD incidence declined from 15.7/100,000 to 12.2/100,000. Incidence of PCV5-type IPD declined after PCV13 introduction (Table), driven primarily by serotypes 19A and 7F. Non-PCV13 serotype IPD incidence did not change. During January 2012-June 2012, 29\% of IPD was due to PCV13 serotypes.

Table: PCV5-type IPD rates (cases per 100,000)

\begin{tabular}{|l|l|l|l|}
\hline Age (years) & $\begin{array}{l}\text { Pre-PCV13 } \\
\text { (July 2004-June 2010) }\end{array}$ & $\begin{array}{l}\text { Post-PCV13 } \\
\text { (July 2011-June 2012) }\end{array}$ & $\begin{array}{l}\text { Percent decrease } \\
(95 \% \mathrm{Cl})\end{array}$ \\
\hline $18-49$ & 2.8 & 1.5 & $65(60-68)$ \\
\hline $50-64$ & 6.8 & 4.5 & $54(51-58)$ \\
\hline$\geq 65$ & 11.7 & 7.8 & $47(39-53)$ \\
\hline
\end{tabular}

Conclusion: PCV13 use among children reduced IPD incidence among adults. The remaining proportion of PCV13type cases suggests that sustained, high vaccination coverage might further increase PCV13 impact in adults.

No conflict of interest

ISPPD-0261

Controlling Pneumococcal Disease Around the Globe

\section{INCIDENCE OF INVASIVE PNEUMOCOCCAL DISEASE IN THE NETHERLANDS AFTER INTRODUCTION OF 7-VALENT AND 10-VALENT PNEUMOCOCCAL CONJUGATE VACCINATION}

M.J. Knol ${ }^{1}$, E.A.M. Sanders ${ }^{2}$, B. Vlaminckx ${ }^{3}$, H.E. De Melker ${ }^{1}$, E. Van der Ende ${ }^{4}$

${ }^{1}$ Center for Infectious Disease control, National Institute for Public Health and the Environment, Bilthoven, Netherlands; ${ }^{2}$ Pediatric Immunology, University Medical Center Utrecht, Utrecht, Netherlands; ${ }^{3}$ Medical Microbiology, St. Antonius hospital, Nieuwegein, Netherlands; ${ }^{4}$ Medical Microbiology, Amsterdam Medical Center, Amsterdam, Netherlands

Background and Aims: In the Netherlands, 7-valent pneumococcal conjugate vaccine (PCV7) was introduced in the national immunization program in June 2006 at 2, 3, 4 and 11 months of age without a catch-up campaign and was replaced by PCV10 in May 2011. Vaccine coverage has been $95 \%$ since introduction. We compared the incidence of invasive pneumococcal disease (IPD) before and after introduction of PCV7 and PCV10.

Methods: Isolates from blood and/or cerebrospinal fluid of patients with IPD from nine sentinel laboratories, covering $25 \%$ of the Dutch population, were serotyped. We compared the serotype-specific IPD incidences in June 2004-May 2006 with those in June 2009-May 2011 and June 2011-June 2013.

Results: Compared to 2004-2006, the PCV7-type IPD incidence declined in $2009-2011$ by $95 \%$ and $71 \%$ and in $2011-$ 2013 by $95 \%$ and $87 \%$ in 0-4 and $\geq 65$ year olds, respectively. In 2009-2011, non-PCV7-type IPD incidence increased by $64 \%$ and $23 \%$ and in $2011-2013$ by $77 \%$ and $40 \%$ in $50-64$ and $\geq 65$ year olds, respectively. Incidence of $19 A$ IPD increased in all age groups in 2009-2011 and this continued in 2011-2013 except for 0-4 year olds. Impact of PCV10 implementation was not detected due to the low number of serotype 1, 5, 7F cases among young children. Conclusion: PCV7 resulted in the eradication of PCV7-type IPD within 5 years after its introduction with no increase of non-PCV7-type IPD in young children. Trends in IPD did not change substantially after introduction of PCV10. 
ISPPD-0390

Controlling Pneumococcal Disease Around the Globe

\title{
AN EARLY REDUCTION OF INVASIVE PNEUMOCOCCAL INFECTIONS AFTER PCV-10 IMMUNISATION
}

\author{
H. Erlendsdóttir ${ }^{1}$, A. Haraldsson ${ }^{2}$, B. Hrafnkelsson ${ }^{3}$, K.G. Kristinsson ${ }^{1}$
}

${ }^{1}$ Clinical Microbiology, Landspitali University Hospital and University of Iceland, Reykjavík, Iceland; ${ }^{2}$ Children's Hospital Iceland, Landspitali University Hospital and University of Iceland, Reykjavík, Iceland; ${ }^{3}$ Mathematics, University of Iceland, Reykjavík, Iceland

Background: Iceland introduced pneumococcal conjugate vaccine (PCV10, Synflorix) in the childhood vaccination program in 2011. Our aim was to monitor changes in the prevalence of invasive pneumococcal infections (IPI) and serotypes following the vaccination.

Material: All IPI in Iceland are recorded at the Department of Microbiology, Landspitali University Hospital. Information on age, mortality and serotypes was gathered for pre- and post vaccine periods, i.e. 2008-2010 (until Oct 15th) and 2011-2013 (until Oct 15th). Information on the population and mortality was obtained from national registries.

Results: A total of 116 and 79 IPI were diagnosed in Iceland in the pre- and post vaccine periods (annual incidence: 12 and $8.2 / 100.000, p=0.007)$. For the same periods the annual incidence in patients $<2$ years of age declined from 73 to $14 / 100.000$, ( $p<0.001)$, from 6.8 to $5.4 / 100.000$ in patients $>2<65(p=0.273)$ and from 36 to $26 / 100.000$ in patients $>65$ years $(p=0.157)$. The proportion of PCV-10 serotypes declined between the two periods $(p<0.001)$; from $71 \%$ to $50 \%$ in $<2$ years of age, $64 \%$ to $36 \%$ in $2-65$ years and $58 \%$ to $36 \%$ in $>65$ years. In the pre vaccine period one child (5\%) and 12 adults patients (14\%) died within one month of the diagnosis, $62 \%$ had PCV-10 serotypes. In the post vaccine period no child and 15 adult patients $(20 \%)$ died, $20 \%$ had PCV-10 serotypes. Conclusion: A rapid decline in IPI was recorded already three years following the vaccine implementation. This was mainly due to a decrease in the youngest children and a decline in PCV10 serotypes overall.

Conflict of interest

ISPPD-0271

Controlling Pneumococcal Disease Around the Globe

\section{MULTICENTER PROSPECTIVE SURVIEILLANCE OF INVASIVE PNUEMOCOCCAL DISEASE IN CHILDREN (<5 YEARS) FROM MAHARASHTRA , INDIA}

S. Lalwani ${ }^{1}$, M. Modak ${ }^{2}$, K. Suryavanshi ${ }^{2}$, S. Palkar ${ }^{3}$, A. Manoharan ${ }^{4}$, A. Sapru ${ }^{5}$, M. Satpute ${ }^{6}$, S. Karande ${ }^{7}$, G. Natara-

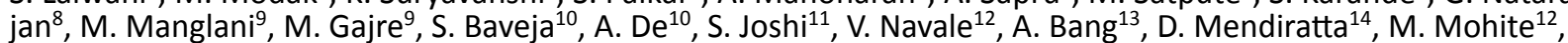
Y. Amdekar' ${ }^{12}$, I. Khosala ${ }^{12}$

${ }^{1}$ Pediatrics, Bharati Vidyapeeth Medical college pune, Pune, India; ${ }^{2}$ Microbiology, Bharati Vidyapeeth Deemed University Medical College, Pune, India; ${ }^{3}$ Pediatrics, Bharati Vidyapeeth Deemed University Medical College, Pune, India; ${ }^{4}$ Infectious Disease, Christian Medical College, Vellore, India; ${ }^{5}$ Pediatrics, KEM Hospital \& Research Center, Pune, India; ${ }^{6}$ Microbiology, KEM Hospital \& Research Center, Pune, India; ${ }^{7}$ Pediatrics, KEM Hospital \& Medical College Parel, Mumbai, India; ${ }^{8}$ Microbiology, KEM Hospital \& Medical College Parel, Mumbai, India; ${ }^{9}$ Pediatrics, Lokmanya Tilak Municipal Corporation Hospital Sion, Mumbai, India; ${ }^{10}$ Microbiology, Lokmanya Tilak Municipal Corporation Hospital Sion, Mumbai, India ${ }^{11}$ Microbiology, Sentinel Laboratory, Mumbai, India; ${ }^{12}$ Pediatrics, Sentinel Site, Mumbai, India; ${ }^{13}$ Pediatrics, Mahatma Gandhi Institute of Medical Sciences Sewagram, Wardha, India; ${ }^{14}$ Microbiology, Mahatma Gandhi Institute of Medical Sciences Sewagram, Wardha, India

Background: Streptococcus pneumoniae is a major vaccine preventable cause of mortality worldwide, including India. At least 93 pneumococcal serotypes are known, but prevalent serotypes \& their antibiogram data from invasive pneumococcal disease (IPD) in Indian children ( $<5$ years) is limited, knowledge of which is crucial for appropriate vaccine selection.

Aim: To identify the serotypes, antibiogram of pneumococcal isolates.

Methods: A prospective hospital and sentinel pediatric, laboratory based Study with central coordinating center was conducted over 2 years at 6 centers as part of Alliance for Surveillance of Invasive Pneumococci (ASIP). Based on WHO case definition, suspected cases of pneumonia, meningitis or bacteremia in $<5$ years children were selected \& enrolled after obtaining informed parent consent. Microbiology workup was based on World Health Organization (WHO)/Centers for Disease Control and Prevention (CDC) surveillance manual. Blood/cerebrospinal fluid/other sterile fluids were cultured. Isolates were serotyped by Quellung technique, antibiogram was done with E-test. Results: Out of 1215 , occurrence of IPD among study subjects was $6 \%$. Total isolates were 73 . Table depicts serotyping results of isolates.

\begin{tabular}{|l|l|}
\hline SEROTYPE & NUMBER OF ISOLATE \\
\hline 5 & 11 \\
\hline 14 & 10 \\
\hline 1 & 7 \\
\hline $19 \mathrm{~F}$ & 6 \\
\hline $10 \mathrm{~F} / 19 \mathrm{~A} / 6 \mathrm{~A} / 6 \mathrm{~B}$ & $16,(4$ isolate of each serotypes $)$ \\
\hline $2 / 23 \mathrm{~F} / 35 \mathrm{~F}$ & $6,(2$ isolate of each serotypes $)$ \\
\hline $21 / 34 / 36 / 15 \mathrm{~B} / 17 \mathrm{~F} / 18 \mathrm{C} / 23 \mathrm{~A} / 18 \mathrm{~F} / 24 \mathrm{~A} / 33 \mathrm{~B} / 7 \mathrm{~B} / 7 \mathrm{C} / 9 \mathrm{~A} / 9 \mathrm{~V}$ & $14,(1$ isolate of each serotypes $)$ \\
\hline \multicolumn{2}{|c|}{ Unable to type-3 } \\
\hline
\end{tabular}


Mortality was about $10 \%$. $>90 \%$ isolates were susceptible to penicillin, cefotaxime but over 60 and $30 \%$ isolates were resistant to trimethoprim-sulphamethoxazole, erythromycin respectively.

Conclusion: Data although limited, is important. Continued surveillance efforts are needed.

No conflict of interest

ISPPD-0053

Controlling Pneumococcal Disease Around the Globe

\section{PEDIATRIC INVASIVE DISEASE POTENTIAL OF PNEUMOCOCCI AT THE 13-VALENT PNEUMOCOCCAL CONJUGATE VACCINE ERA}

E. Varon ${ }^{1}$, C. Levy ${ }^{2}$, S. Béchet ${ }^{3}$, C. Doit ${ }^{4}$, R. Cohen ${ }^{2}$

${ }^{1}$ Microbiology, cnrp, Paris, France; ${ }^{2}$ Epidemiology, Activ Gpip, St Maur, France; ${ }^{3}$ Epidemiology, Activ, St Maur, France; ${ }^{4}$ Microbiology, Robert Debré Hosp, Paris, France

Background: Changes in serotypes (STs) distribution have been observed, particularly following the pneumococcal conjugate vaccines (PCVs) implementation, and uncommon non vaccine STs so far are now circulating. There is a need to distinguish among these latter, those with higher invasive disease potential.

Methods: Invasive pneumococcal disease (IPD) STs isolated from children 6 to 24 months were compared with nasopharyngeal (NP) colonizing STs. To assess the invasive potential of a given ST, odds ratio (OR) was calculated. For each ST, OR $>1$ indicated an increased probability to be associated with IPD, while OR<1 indicated a decreased probability. Pneumococcal strains were conventionally serotyped with antisera from Statens Seruminstitute.

Results: Between 2011 and 2012, the pooled dataset included pneumococci isolated from 189 healthy carriers and 567 IPD (among which 143 meningitis and 73 pneumonia). Mean age \pm SD was 12.9 months \pm 5.1 (median 12.0) without any difference between the groups (healthy carriers and IPD). ST7F and ST19A were positively associated to IPD, meningitis and pneumonia with the highest ORs found for ST7F. ST24F was positively associated to IPD and meningitis, but not to pneumonia. Conversely, the lowest significant OR (0.1) was observed for ST23B in IPD and meningitis, and for ST15A in pneumonia. For two major invasive STs, case/carrier ratios were not calculated since no carrier was recorded (ST12F, ranking at the 2 nd place in all invasive diseases and ST1, ranking at the 4th place for pneumonia).

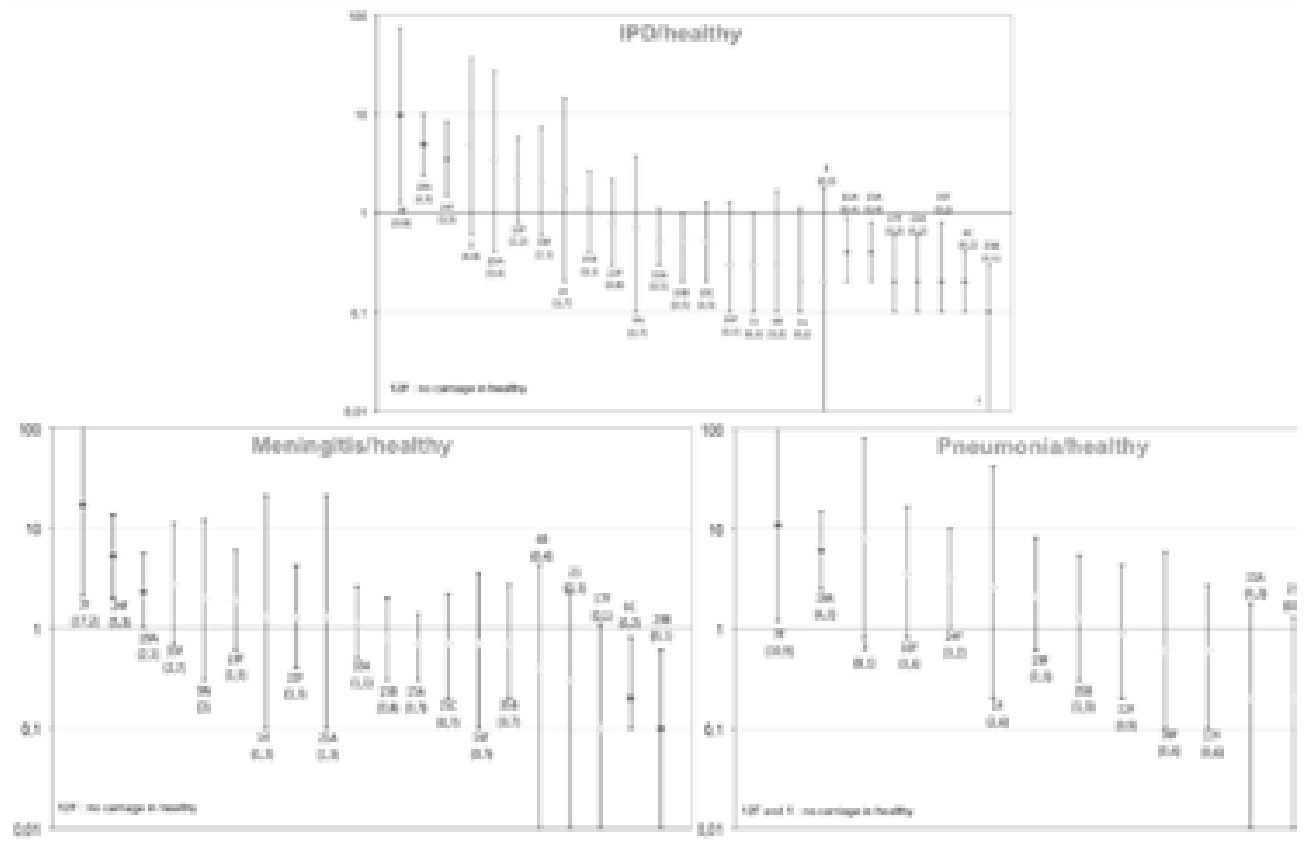

Conclusion: Our results suggest that $24 \mathrm{~F}$ had the most important invasive disease potential among the non vaccine STs. Conversely ST 11A, 15A, 17F, 23A, 35F, 6C and 23B were poorly associated with IPD but for most of the other non vaccine STs, the risk was not assessed.

No conflict of interest 
ISPPD-0052

Controlling Pneumococcal Disease Around the Globe

\title{
PNEUMOCOCCAL MENINGITIS CAUSED BY VACCINE SEROTYPES IN PNEUMOCOCCAL CONJUGATE VACCINATED CHILDREN IN FRANCE
}

\author{
C. Levy ${ }^{1}$, E. Varon², S. Béchet ${ }^{3}$, S. Bonacorsi ${ }^{4}$, R. Cohen ${ }^{1}$, G.P.I.P. Groupe des Pédiatres et Microbiologistes ${ }^{5}$ \\ ${ }^{1}$ Epidemiology, Activ Gpip, St Maur, France; ${ }^{2}$ Microbiology, cnrp, Paris, France; ${ }^{3}$ Epidemiology, Activ, St Maur, France; ${ }^{4}$ Microbiology, Robert De- \\ bré Hosp, Paris, France; ${ }^{5}$ Epidemiology, Activ, Paris, France
}

Background: In 2002, the 7-valent pneumococcal conjugate vaccine (PCV7) was recommended in France, followed by PCV13 since June 2010. In order to describe the characteristics of vaccinated children with pneumococcal meningitis (PM) caused by PCV serotypes (STs), GPIP/ACTIV set up an active surveillance network.

Methods: From 2001 to 2013, 227 pediatric wards enrolled children with PM. Risk factors, PCV vaccination status (analysed according to French recommendations), and case fatality rate were recorded. Pneumococcal strains were conventionally serotyped with antisera from Statens Serum Institute.

Results: Among the 1441 PM reported during 13 years we identified, in PCV vaccinated children, 40 PM caused by PCV STs (PCV7, $n=30,6$ additional STs of PCV13, $n=10$ ). Among the 25 children who had received at least one PCV7 dose before PM caused by PCV7 ST, 12 were incompletely vaccinated according to the age, and 13 fulfilled the criteria of vaccine failure: ST $4(n=1), 23 F(n=1), 14(n=2), 6 B(n=3)$, and $19 F(n=6)$. Among the 15 children who had received at least one PCV13 dose before PM caused by PCV13 ST, 8 were incompletely vaccinated and 7 fulfilled the criteria of vaccine failure: ST $19 F(n=3)$, and $19 A(n=4)$.

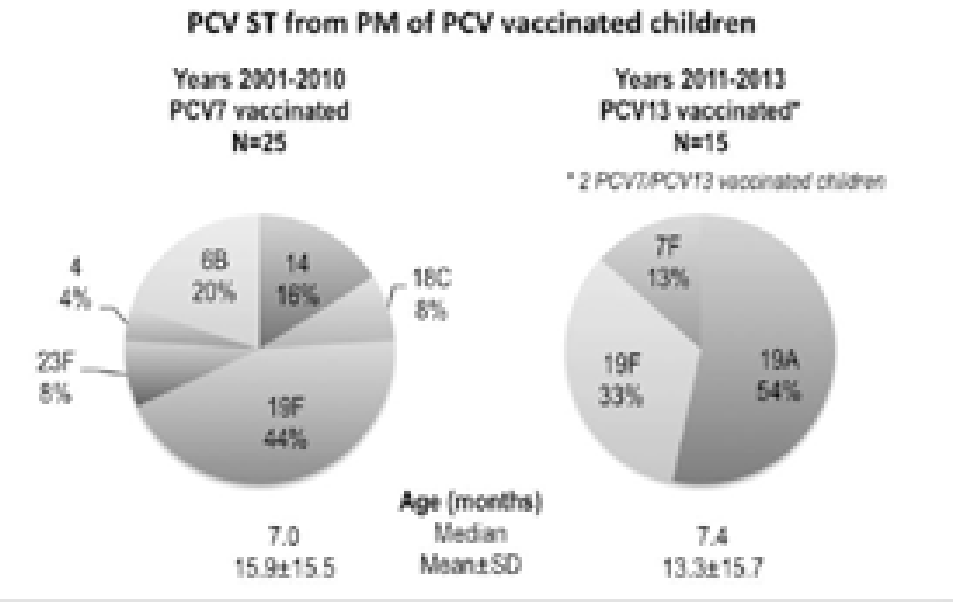

Conclusion: During 13 years, 1.4\% of PM caused by PCV ST occurred in PCV vaccinated children (adequately for age). Since PCV13 implementation, we observed, in vaccinated children, the disappearance of PCV7 STs except 19F, which represented $40 \%$ of overall cases.

No conflict of interest

ISPPD-0187

Controlling Pneumococcal Disease Around the Globe

\section{REDUCED HOSPITALIZATION DUE TO SINUSITIS AND PNEUMONIA AFTER INTRODUCTION OF PCV7}

A. Lindstrand ${ }^{1}$, R. Bennet ${ }^{2}$, I. Galianis ${ }^{3}$, M. Blennow ${ }^{4}$, L. Schollin Ask ${ }^{4}$, S. Hultman Dennisson ${ }^{5}$, M. Ryd Rinder ${ }^{4}$, M. Eriksson ${ }^{6}$, B. Henriques Normark ${ }^{7}$, A. Örtqvist ${ }^{8}$, T. Alfvén ${ }^{9}$

${ }^{1}$ Vaccine Unit, Swedish Institute for Communicable DIsease Control, Solna, Sweden; ${ }^{2}$ Astrid Lindgren Children's Hospital, Karolinska University Hospital, Solna, Sweden; ${ }^{3}$ Epidemiology, Swedish Institute for Communicable Disease Control, Solna, Sweden; ${ }^{4}$ Sachs' Children's Hospital, South General Hospital, Stockholm, Sweden; ${ }^{5}$ Otorhinopharyngeal department, Karolinska University Hospital, Stockholm, Sweden; ${ }^{6}$ Astrid Lindgren Children's Hospital, Karolinska University Hospital, Stockholm, Sweden; ${ }^{7}$ Department of Microbiology Tumor and Cell Biology, Karolinska Institutet, Stockholm, Sweden; ${ }^{8}$ Department of Communicable Disease Control and Prevention, Stockholm County Council, Stockholm, Sweden; ${ }^{9}$ Sachs' Children's Hospital, South General Hospital Stockholm Sweden, Stockholm, Sweden

Background: Streptococcus pneumoniae is the major cause of bacterial pneumonia and sinusitis. S. pneumoniae kills about 1.3 million children $<5$ years annually and sinusitis is a feared paediatric disease due to orbital and intracranial complications. Although effective against invasive pneumococcal disease, the effectiveness of pneumococcal conjugate vaccine (PCV) against pneumonia is less consistent and its effect on sinusitis is not known. Here, we compared hospitalizations rates due to sinusitis and pneumonia 4 years before and after introduction of PCV7 in 2007.

Method: Retrospective study of hospital registry data on hospitalizations due to sinusitis and pneumonia in all children $0-<18$ years. Trend analysis, incidence rates and relative risks were calculated comparing July 2003-June 2007 to July 2008-June 2012, excluding the year of PCV7 introduction.

Results: Sinusitis hospitalizations decreased significantly in children $0-<2$ years from 70 cases/100 000/year before the introduction of PCV7 to 25 cases/100 000/year ( $R R=0.35, P<0.001)$ after the introduction (Fig 1). Pneumonia 
hospitalizations decreased significantly in children $0-<2$ years $(R R 0.81, P<0,001)$ and $2-<5$ years $(R R=0.85, P=0.002)$ during the same period (Fig 2).

Conclusion: Introduction of PCV led to a decreased risk of hospitalization due to sinusitis (65\% decreased risk) and to pneumonia ( $19 \%$ decreased risk) in children $0-<2$ years.

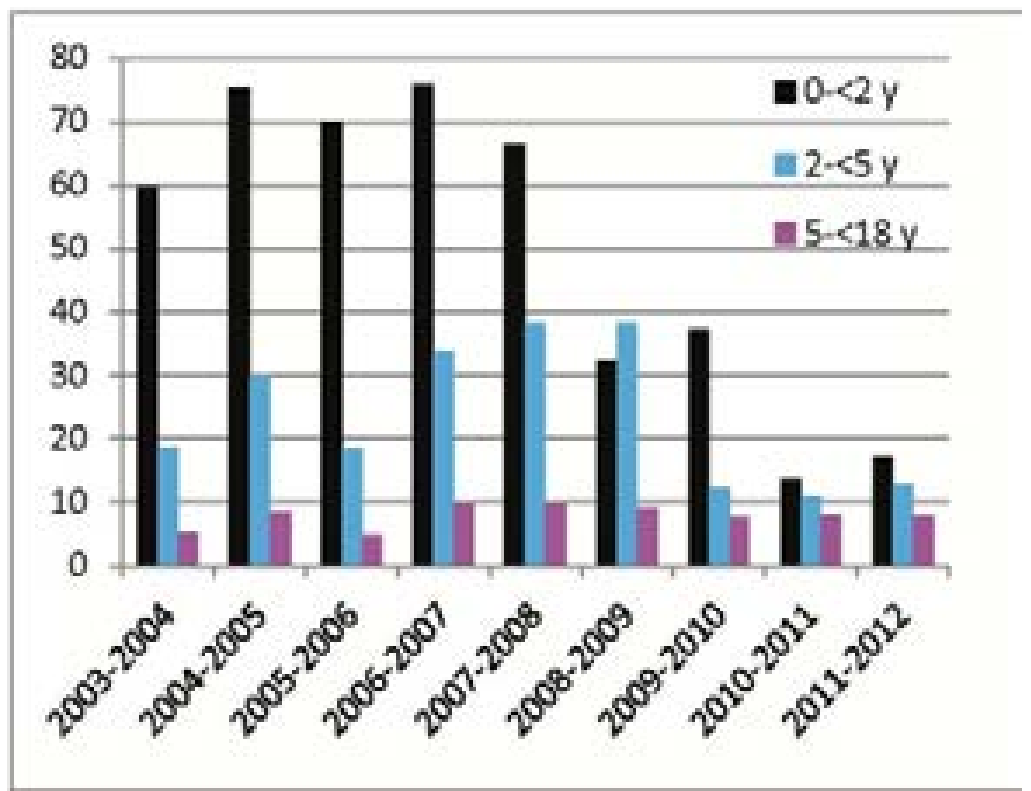

Fig 1 Incidence hospitalisations due to sinusitis /100000 children age $0-<2$ y

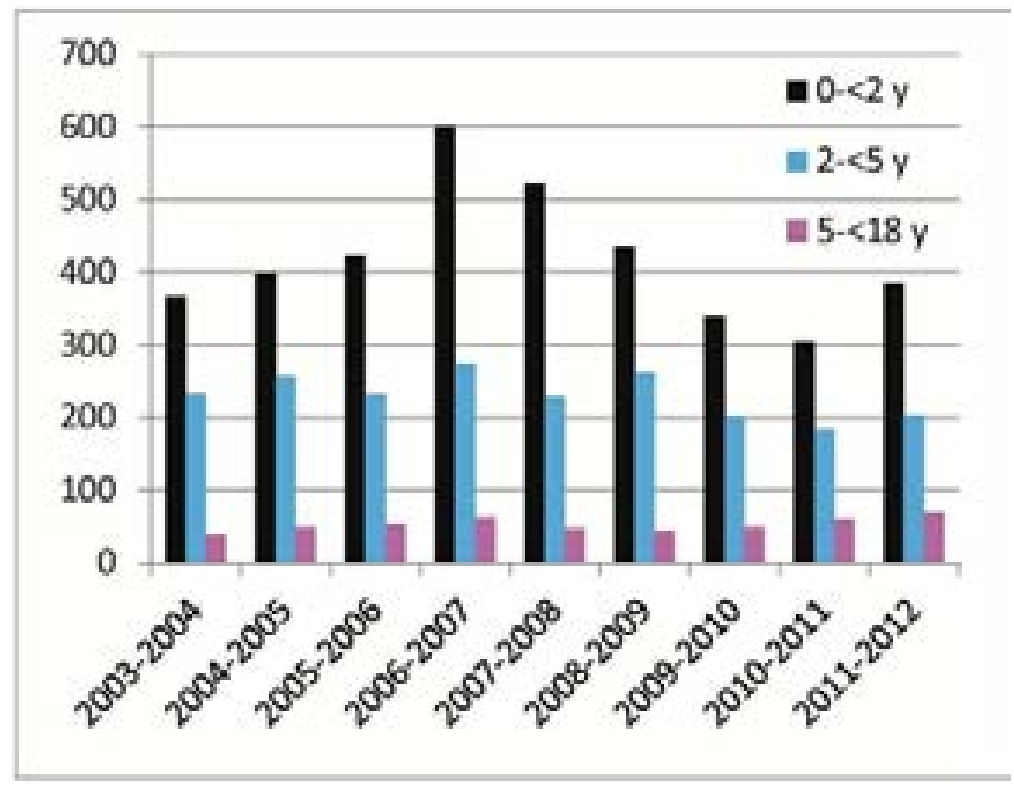

Fig 2 Incidence hospitalisations due to bacterial pneumonia /100000 children age $0-<2$ y

No conflict of interest 
ISPPD-0351

Controlling Pneumococcal Disease Around the Globe

\section{IMPACT OF INTRODUCTION OF PCV-13 ON HOSPITALIZATIONS IN ROSARIO-ARGENTINA. PRELIMINARY RESULTS FOR INVASIVE PNEUMOCOCCAL DISEASE (IPD) FOLLOWING THE FIRST YEAR}

S. Lopez Papucci ${ }^{1}$, A. Badano ${ }^{2}$, E. Bonifachich ${ }^{3}$, M. Alvarez ${ }^{1}$, A. Chiossone ${ }^{1}$, M. Arburu ${ }^{4}$, G. Ensinck ${ }^{1}$, A. Uboldi $^{5}$, S. Fossati $^{6}$, M. Regueira ${ }^{6}$

${ }^{1}$ Infectious diseases, V. J. Vilela Children Hospital, Rosario, Argentina; ${ }^{2}$ Laboratory of Microbiology, V. J. Vilela Children Hospital, Rosario, Argentina; ${ }^{3}$ Neumonology, V. J. Vilela Children Hospital, Rosario, Argentina; ${ }^{4}$ Radiology, V. J. Vilela Children Hospital, Rosario, Argentina; ${ }^{5}$ Ministry of Health, Immunization program, Santa Fe, Argentina; ${ }^{6}$ National Institute for Infectious diseases, Microbiology, Buenos Aires, Argentina

Background and Aims: In January 2012 PCV13 was introduced in Argentina using 2+1 schedule and catch-up program until 2 years of age. In 2012 vaccination coverage among infants $<1$ year of age was $89 \%$ and $59 \%$ for the first and the second dose, and among children 1 year old $41,8 \%$ and $28,3 \%$, in Rosario city. We compared IPD epidemiology among hospitalized children 4 years prior to, with the transition year post PCV-13 introduction. Methods: Prospective observational study of laboratory confirmed IPD in hospitalized children younger than 13 years carried out from 2008 to 2011 vs 2012. We compared age group-specific, syndrome-specific and serogroupspecific IPD in both periods.

Results: In children $<2$ years there was a trend toward reduction of hospitalizations for pneumococcal pneumonia, but statistical significance was yet not achieved (54.2\% reduction from 68.9 to 31.5 cases $/ 10^{4}$ discharges, $p=$ $0.1087)$. This rate decreased no significantly $66.1 \%$ from 55.2 to 18.7 cases $/ 10^{4}$ discharges in infants younger than 12 months of age $(p=0.146)$ and $40.4 \%$ from 97.4 to 58 cases $/ 10^{4}$ in children $12-23$ months $(p=0.606)$. A nonsignificant $30.14 \%$ reduction in meningitis was seen in children younger than 2 years. Only one infant with IPD due to a vaccine serotype with 1 dose of vaccine and no one with 2 doses, were identified. No difference in hospitalizations for IPD was observed in older children.

Conclusion: Just one year after introduction of PCV-13 in Argentina the hospitalization rates for pneumococcal pneumonia and meningitis show promisory decreases in children younger than 2 years old in Rosario.

Conflict of interest

ISPPD-0303

Controlling Pneumococcal Disease Around the Globe

\section{PNEUMOCOCCAL SEROTYPES ASSOCIATED WITH THE HOMELESS}

T. Marrie ${ }^{1}$, M. Lovgren², C. Mangan 3 , Q. Ibrahim4, D. Hurteau², G. Tyrrell ${ }^{5}$

${ }^{1}$ Faculty of Medicine, Dalhousie University, Halifax, Canada; ${ }^{2}$ Bacterial Typing Unit, Provincial Laboratory for Public Health, Edmonton, Canada

${ }^{3}$ Undergraduate Medical Education, UAlberta, Edmonton, Canada; ${ }^{4}$ EPICORE, UAlberta, Edmonton, Canada; ${ }^{5}$ Laboratory Medicine and Pathology, UAlberta, Edmonton, Canada

Background: The homeless state places individuals in living conditions that can result in a greater disposition to a variety of infectious diseases. Invasive pneumococcal disease (IPD) is one such disease that is becoming more recognized as being associated with the homeless population. Surveillance of IPD in Alberta has been performed by the Provincial Laboratory for Public Health (Provlab) since 2000. One of the metrics captured during this surveillance is homelessness. We report on the association of specific pneumococcal serotypes with the homeless in Alberta. Methods: IPD is a notifiable disease to Provincial Health Authorities. As such, the isolates from all cases of IPD are forwarded to the Provlab for serotyping. Cases of IPD identified through isolate submission were followed up by a study nurse to determine the cases place of residence.

Results: Analysis of pneumococcal serotypes from 2000 to 2012 found an association of specific serotypes with the homeless population. Of 57 serotypes identified among over 5600 isolates, five serotypes accounted for $57 \%$ of invasive pneumococcal disease (IPD) from homeless people. These serotypes were 5, 8, 9N, $12 \mathrm{~F}$ and 20 . These five serotypes only made up $19 \%$ of serotypes found in cases of IPD from the non-homeless population. We also noticed temporal clustering. Serotype 20 was found in elevated numbers from 2011 to 2013 and serotype 5 from 2005 to 2008.

Conclusion: Specific pneumococcal serotypes cause the majority of IPD in the homeless population. In addition, the temporal clustering events suggest successive outbreaks caused by these serotypes in this population.

Conflict of interest 
ISPPD-0331

Controlling Pneumococcal Disease Around the Globe

\title{
SEROTYPE EVOLUTION AND ANTIMICROBIAL SUSCEPTIBILITY OF IPD PRE AND POST INTRODUCTION OF 13-VALENT PNEUMOCOCCAL CONJUGATE VACCINE (PCV13) IN GREECE
}

V. Syriopoulou ${ }^{1}$, G.A. Syrogiannopoulos ${ }^{2}$, D. Menegas ${ }^{3}$, E. Koutouzis ${ }^{1}$, T. Georgakopoulou ${ }^{1}$, J. Kavaliotis ${ }^{4}$, E. Galana$\mathrm{kis}^{5}$, A. Siamopoulou-Mavridou ${ }^{6}$, A. Tsilimigaki ${ }^{7}$, T. Merides $^{8}$, E. Roilides ${ }^{9}$, G.L. Daikos ${ }^{10}$, I.N. Grivea ${ }^{2}$, M. Tsolia ${ }^{11}$

${ }^{1}$ First Department of Pediatrics University of Athens, Aghia Sophia Children's Hospital, Athens, Greece; ${ }^{2}$ Department of Pediatrics University of Thessaly, Medical School, Larissa, Greece; ${ }^{3}$ Medical Department, Pfizer Hellas, Athens, Greece; ${ }^{4}$ Pediatric Department, Thessaloniki Hospital of Infectious Diseases, Thessaloniki, Greece; ${ }^{5}$ Department of Pediatrics, University of Crete, Heraklion, Greece; ${ }^{6}$ Child Health Department, University of loannina, loannina, Greece; ${ }^{7}$ Second Pediatric Department, Venizelion General Hospital, Heraklion, Greece; ${ }^{8}$ Department of Pediatrics, General Hospital of Volos, Volos, Greece; ${ }^{9}$ Third Dpt of Pediatrics, Aristotle University of Thessaloniki, Thessaloniki, Greece; ${ }^{10}$ First Department of Propedeutic Medicine University of Athens, Laiko General Hospital, Athens, Greece; ${ }^{11}$ Second Department of Pediatrics University of Athens, P. and A. Kyriakou Children's Hospital, Athens, Greece

Background: The aim was to examine the evolving serotype epidemiology and antimicrobial susceptibility of Streptococcus pneumoniae isolates causing invasive pneumococcal disease (IPD) in children $\leq 14$ y old following introduction of PCVs to the Greek National Immunization Programme (NIP).

Methods: Data from a 4 year prospective study conducted between September 2008 and October 2012 in 10 pediatric hospitals are presented. Serotyping was performed by latex agglutination and Quellung reaction using anti-sera (SSI, Denmark). Antimicrobial susceptibility was determined by E-test.

Results: Among 177 isolates collected ( $37.2 \% \leq 2$ y old) a $38 \%$ decrease of overall IPD cases was observed between pre-PCV13 (2008-2010) and post-PCV13 (2011-2012) periods; reaching $47.6 \%$ in children $\leq 2$ y old. The decrease was more notable in meningitis cases $(71.4 \%)$ followed by bacteremia $(41.7 \%)$ and bacteremic pneumonia $(26.3 \%)$ cases. The commonest IPD serotypes in the pre-PCV13 era were 7F (23.2\%), 19A (20.2\%), 1 (11.1\%) and $3(8.0 \%)$ while 1.5 years post-PCV13 were $7 F$ and $19 A$ (25.8\% each) and 24F (9.7\%). PCV7 serotypes became extinct while a substantial decrease (43.8\%) of the 6 additional serotypes in PCV13 was recorded along with NVTs remaining at constant numbers. Resistance to penicillin (resistant or intermediate) exhibited $19.4 \%$ of isolates in the pre-PCV13 period vs. $9.7 \%$ in the post-PCV13 period. However, resistant isolates to erythromycin increased from $15.2 \%$ to $25.8 \%$ between the two periods.

Conclusion: IPD cases decreased significantly after the introduction of PCV13 in the Greek NIP. Further reduction of residual burden of disease can be anticipated with the achievement of better compliance to the existing schedule.

Conflict of interest

ISPPD-0008

Controlling Pneumococcal Disease Around the Globe

\section{EPIDEMIOLOGY, SEROTYPE DISTRIBUTION, AND ANTIMICROBIAL RESISTANCE OF STREPTOCOCCUS PNEUMONIAE IN KUWAIT: AN 8-YEAR STUDY OF PNEUMOCOCCAL CONJUGATE VACCINE IMPACT}

\author{
E. Mokaddas ${ }^{1}$, M. Albert ${ }^{1}$ \\ ${ }^{1}$ Microbiology, Faculty of Medicine, Dasma, Kuwait
}

Background and Aim: The 7-valent polysaccharide-protein conjugate pneumococcal vaccine (PCV7) was introduced in Kuwait in August 2006, and the PCV13 in August 2010, for children aged <2 years.

Objectives: To evaluate the impact of PCV7 and PCV13 on epidemiology, serotype distribution, and antimicrobial resistance of all Streptococcus pneumoniae isolates and the vaccine coverage rate in all age groups.

Methods: The study included all cases of invasive and non-invasive pneumococcal disease (IPD) in children and adults among all age groups from January 2004 to December 2011. The isolates were serotyped using Quellung reaction antisera and their susceptibility to penicillin was determined using the Etest method.

Results: 645 pneumococcal isolates were included in the study. From January 2004 till July 2006, there were 250 isolates ( $22 \%$ were invasive and $27 \%$ were from children $\leq 5$ years). From August 2006 till December 2011, there were 390 isolates ( $32 \%$ were invasive $s$ and $35 \%$ were from children $\leq 5$ years). The predominant invasive serotypes from 2004-2006 were 19F, 23F, 9V and 14, while from 2006-2011 were 19F, 8, 6A, 9V, 14, 3, 1, 19A, 5 and 15B. The percentage serotype coverage by PCV7 in children $<2$ years dropped from $75.0 \%$ in the first period to $34.6 \%$ in the second period. A positive impact of PCV7 on the incidence of IPD and drop in penicillin resistance was demonstrated in both children $<5$ years and adults $>50$ years.

Conclusion: With the appearance of non-PCV7 pneumococcal serotypes, broader serotype coverage vaccines are needed for the prevention of IPD.

No conflict of interest 
ISPPD-0454

Controlling Pneumococcal Disease Around the Globe

\title{
REDUCTION OF INVASIVE PNEUMOCOCCAL DISEASE THREE YEARS AFTER INTRODUCTION OF THE 13 VALENT CONJUGATE VACCINE IN THE OXFORDSHIRE REGION, ENGLAND
}

\author{
C.E. Moore ${ }^{1}$, A.S. Walker ${ }^{1}$, J. Paul ${ }^{2}$, D. Foster ${ }^{1}$, S. Mahar ${ }^{1}$, D. Griffiths ${ }^{1}$, K. Knox ${ }^{1}$, T.E. Peto ${ }^{1}$, D.W. Crook ${ }^{1}$ \\ ${ }^{1}$ Nuffield Department of Medicine, University of Oxford, Oxford, United Kingdom; ${ }^{2}$ Microbiology Royal Sussex County Hospital, Public Health Eng- \\ land, Brighton, United Kingdom
}

Background: The impact of the 7-valent pneumococcal conjugate vaccine on invasive pneumococcal disease (IPD) is well described, but few reports exist on the additional impact of the 13-valent vaccine.

Methods: We calculated IPD incidence across all ages in a surveillance project in Southern England following implementation in under twos of the 7-valent (September 2006) and 13-valent (April 2010) conjugate vaccines (nine hospitals from 1996, one from 2005, one from 2009; 4935 cases).

Results: Overall incidence decreased from 10/100,000 persons per year in 1996/1997 to 7/100,000 in 2012/2013. Declines were greater in under two's (from 37/100,000 to 14/100,000 respectively). IPD with 7-valent vaccine serotypes decreased in under and over twos after its introduction $(p<0.001)$. However, incidence of IPD with the additional six serotypes in the 13-valent vaccine and non-vaccine types (NVT) increased in over two's (both $p<$ 0.001 ,) with similar non-significant increases in under two's ( $p=0.16, p=0.18$ respectively). IPD with the additional six PCV13 serotypes declined significantly after PCV13 introduction in over two's $(p<0.001)$ and under two's ( $p$ $=0.007)$ whilst IPD with NVT remained unchanged in both $(p>0.25)$. Overall incidence for 2011-13 with PCV7, additional six PCV13 serotypes and NVT was $0.3,2.8$ and 4.4/100,000 persons overall, and 0.9, 2.4 and 10.8/100,000 person years under two.

Conclusion: IPD incidence has decreased to 1-2/100,000 population for the target group (children $<2$ years) for 7 -valent and 13-valent vaccine serotypes. However, non-vaccine type IPD incidence has increased to 11/100,000 in this age group, and 5/100,000 overall, indicating the importance of ongoing surveillance and vaccine extension.

No conflict of interest

ISPPD-0329

Controlling Pneumococcal Disease Around the Globe

\begin{abstract}
CASE-CONTROL STUDY OF EFFECTIVENESS OF 13-VALENT PNEUMOCOCCAL CONJUGATE VACCINE (PCV13) AGAINST INVASIVE PNEUMOCOCCAL DISEASE (IPD) IN THE U.S.: PRELIMINARY ESTIMATES
\end{abstract}

M. Moore ${ }^{1}$, R. Link-Gelles ${ }^{1}$, R. Guevara ${ }^{2}$, J. Rosen $^{3}$, M. Barnes ${ }^{4}$, S. Petit ${ }^{5}$, C. Holtzman ${ }^{6}$, R. Lynfield ${ }^{6}$, W. Schaffner ${ }^{7}$, L. Harrison ${ }^{8}$, S. Currenti ${ }^{9}$, M. Dimond ${ }^{10}$, A. Reingold ${ }^{11}$, M.M. Farley ${ }^{12}$, S. Harb ${ }^{12}$, K. Scherzinger ${ }^{13}$, A. Thomas ${ }^{14}$, L. Mc$\mathrm{Gee}^{1}$, B. Beall ${ }^{1}$, C. Whitney ${ }^{1}$

${ }^{1}$ Respiratory Diseases Branch, Centers for Disease Control \& Prevention, Atlanta, USA; ${ }^{2}$ Emergency Preparedness and Response Program, LA County Department of Public Heatlh, Los Angeles, USA; ${ }^{3}$ Immunization Program, New York City Dept of Health \& Mental Hygiene, New York, USA; ${ }^{4}$ Emerging Infections Program, Colorado Dept of Public Health and Environment, Denver, USA; ${ }^{5}$ Emerging Infections Program, Connecticut Department of Public Health, Hartford, USA; ${ }^{6}$ Emerging Infections Program, Minnesota Department of Health, St. Paul, USA; ${ }^{7}$ Department of Preventive Medicine, Vanderbilt Univeristy, Nashville, USA; ${ }^{8}$ Department of International Health, Johns Hopkins Univeristy, Baltimore, USA; ${ }^{9}$ Emerging Infections Program, New York State Department of Health, Albany, USA; ${ }^{10}$ Division of Disease Control and Prevention, Utah Department of Health, Salt Lake City, USA; ${ }^{11}$ School of Public Health, University of California, Berkeley, USA; ${ }^{12}$ Atlanta Veterans Administration Medical Center, Emory University, Atlanta, USA; ${ }^{13}$ Emerging Infections Program, University of New Mexico, Albuquerque, USA; ${ }^{14}$ Emerging Infections Program, Oregon Public Health Division, Portland, USA

Background and Aims: PCV13 was introduced for U.S. children $<5$ years in February 2010. We conducted a casecontrol study of vaccine effectiveness (VE).

Methods: IPD cases had sterile-site isolation of pneumococcus from 2-59 month-old children residing in Los Angeles County, New York City, Utah, or any of 10 Active Bacterial Core surveillance sites. Controls selected from birth certificates were matched to cases based on age +/- 2 weeks and postal code of residence. Serotyping was performed by reference laboratories and vaccination status was established through review of medical records. VE was estimated as 1 minus the matched odds ratio (mOR) of vaccination among cases vs. controls, multiplied by $100 \%$.

Results: During May 2010-May 2013, we enrolled 765 (72\%) cases among 1,066 eligible IPD episodes (median age 23 months [range 2-59]). Among 2274 controls, coverage with $>1$ dose increased from 37\% in June-November 2010 to $96 \%$ in December 2012-May 2013. Overall, 224 (29\%) of enrolled cases had PCV13 serotypes and 209 (93\%) of these were caused by serotypes 19A, 7F, and 3. No enrolled cases had IPD caused by serotypes 1 or 5 . VE of $>1$ dose against all IPD, PCV13 serotypes, and serotypes 19A, 7F, and 3 (collectively) was $65 \%(95 \% \mathrm{Cl} 52-75), 89 \%(95 \% \mathrm{Cl}$ $79-95)$, and $89 \%$ (95\% Cl 79-95), respectively. After excluding serotype $3, \mathrm{VE}$ of $>1$ dose against serotypes $19 \mathrm{~A}$ and $7 \mathrm{~F}$ was $93 \%(95 \% \mathrm{Cl} 82-97)$.

Conclusion: Based on these preliminary estimates, PCV13 is highly effective against IPD in young children, especially serotypes 19A and 7F.

No conflict of interest 
ISPPD-0444

Controlling Pneumococcal Disease Around the Globe

\title{
IMPACT OF 13-VALENT PNEUMOCOCCAL CONJUGATE VACCINE ON A POPULATION AT HIGH RISK FOR INVASIVE PNEUMOCOCCAL DISEASE
}

\author{
K.L. Mosso ${ }^{1}$, L.R. Grant ${ }^{1}$, R.C. Weatherholtz ${ }^{1}$, J. Campbell ${ }^{1}$, C. Donaldson ${ }^{1}$, J. Dallas ${ }^{1}$, R. Reid ${ }^{1}$, M. Santosham ${ }^{1}$, \\ L.L. Hammitt ${ }^{1}$, K.L. O’Brien ${ }^{1}$ \\ ${ }^{1}$ Center for American Indian Health, Johns Hopkins School of Public Health, Baltimore MD, USA
}

Background: Historically high rates of invasive pneumococcal disease (IPD) among Native Americans living on the Navajo Nation dramatically declined after pneumococcal conjugate vaccine (PCV7) introduction in 2000. In April 2010, 13-valent PCV (PCV13) replaced 7-valent PCV. We describe the IPD epidemiology in all ages during the PCV13 early routine-use era (September 2010 forward).

Methods: Pneumococcal isolates cultured from normally sterile sites in Navajo individuals were identified through active, laboratory-based surveillance. Isolates were serotyped by Quellung; 6A and 6C were distinguished by PCR. Indian Health Service User Population denominators were used for IPD rate calculations. IPD rates from 36-month periods before (2007-2009) and after (September 2010 - August 2013) PCV13 introduction were compared.

Results: We identified 372 IPD cases before and 251 after PCV13 introduction, a 33\% reduction in average annual cases. The PCV13-type IPD rate in children aged $<2$ years declined from 107 to 13 cases $/ 100,000(p<0.001)$. Rates of IPD for serotypes 7F (50 vs. 4 cases/100,000; $p=0.001$ ) and 19A (39 vs. 0 cases $/ 100,000 ; p=0.001$ ) decreased significantly; non-significant reductions occurred in this age group for serotypes 1 ( 7 vs. 0 cases $/ 100,000 ; p=0.26$ ), $19 \mathrm{~F}$ and $6 \mathrm{C}$ ( 4 vs. 0 cases per 100,000 each; $p=0.51$ ). Among adults, the PCV13-type IPD rate declined from 26 to 16 cases $/ 100,000(p<0.001)$. The rate of non-vaccine type IPD remained unchanged in all ages.

Conclusion: PCV13-IPD rates among children and adults declined significantly following PCV13 introduction. Further surveillance is necessary to account for secular trends, to confirm PCV13 indirect effects and further measure serotype specific impact.

No conflict of interest

\section{INCIDENCE OF INVASIVE PNEUMOCOCCAL DISEASE IN WEST UKRAINE REGION DURING LAST 10 YEARS}

O.B. Nadraha ${ }^{1}$

${ }^{1}$ Pediatric Infection Diseases, Lviv National Medical University, Lviv, Ukraine

Background and Aims: World Health Organization (WHO) report that $>1$ million children's deaths annually are due to pneumococcal infection. Invasive pneumococcal disease (IPD) presents with high mortality in children less than 5 years of age, particularly in countries where anti-pneumococcal immunization has not been implemented as part of the national immunization schedule. In Ukraine vaccination with PCV is still available only for children with high risk of developing IPD patients and at some private hospitals.

Methods: A retrospective chart review was conducted of 74 patients (1-17 y old) with cultural confirmed IPD. All patients were treated during 2003-2012 in Lviv Regional Infection Diseases Hospital.

Results: Annual incidence rate of IPD in our region was 9.4 cases/100,000/year. The median of age of patients was 3.2 years. Bacterial pneumonias (27.1\%), purulent meningitis $(24.5 \%)$ and septicemias $(16.6 \%)$ were the most frequent diagnoses. Mortality rate was $7.0 \%$ for IPD and $12.5 \%$ for pneumococcal meningitis. Long-term effects were reported in $12.0 \%$ of IPD cases. Serotypes were identified in $64.8 \%$, and the leading was serotype 1 (20 cases), followed by serotypes 14 ( 17 cases) and 6B (11 cases).

Conclusion: The rate pneunococcal disease in West region of Ukraine is still high. Prevaccination data on disease severity and outcome of IPD are important to determine the impact of vaccination strategies. The pneumoccocal vaccine must be included in national vaccination program.

No conflict of interest

ISPPD-0394

Controlling Pneumococcal Disease Around the Globe

\section{STREPTOCOCCUS PNEUMONIAE; SEROTYPES FROM A PAEDIATRIC COHORT IN A TERTIARY CARE CENTRE}

\author{
S. Nagaraj ${ }^{1}$, B.S. Kalal ${ }^{1}$, S.M. Zia ${ }^{1}$, A. Shet ${ }^{2}$, A. Manoharan ${ }^{3}$ \\ ${ }^{1}$ MICROBIOLOGY, St. John's Medical College, Bangalore, India; ${ }^{2}$ Paediatrics, St. John's Medical College, Bangalore, India; ${ }^{3}$ BMPLIII Medi- \\ cine Unit I \& Infectious Diseases, Christian Medical College, Vellore, India
}

Background and Aims: In the present study we evaluated clinical profile, antimicrobial susceptibility, prevalent serotypes of pneumococcal isolates from children with suspected invasive pneumococcal disease (IPD) admitted to a tertiary care hospital.

Methods: Hospitalized children, $\leq 5$ years with fever $\left(>38^{\circ} \mathrm{C}\right)$; increased respiratory rate or neurological symptoms were recruited,(as part of ASIP project 2011-2013). Ethical Approval was obtained. Identification of pneumococcal isolates from blood (Bactec) or cerebrospinal fluid (CSF) samples was done by routine culture methods and 
confirmed by multiplex -PCR. Confirmed isolates were analysed for antimicrobial susceptibility (MIC) and serotyping by Quellung's test.

Results: Out of the 171 samples received in the lab, 17 grew pneumococci identified standard methods. Fourteen of them were confirmed by Multiplex - PCR. Maximum recruitment were seen in months of January and February $(36.4 \%, 28.6 \%)$. Average age was 21 months. Common clinical feature were of pneumonia (42.8\%) which included cough $(78.5 \%)$, tachypnoea (42.8\%), chest retraction (23.1\%), auscultatory crepitations (38.4\%). Radiological analysis showed consolidation and infiltration in 38.50. Prior antibiotics in the last 48 hours were given in $23.1 \%$ of children. Two isolates belonging to $19 \mathrm{~F}$ and $19 \mathrm{~B}$ serotype were resistant to penicillin. Serotype distribution was $6 \mathrm{~B}$ and $19 \mathrm{~F}$ (2 each) and 1, 2, 6A, 9V, 10A, 14, 15A, 19B, 21 and 35F (1 each).

Conclusion: Based on the serotyped isolated from our paediatric cohort with IPD, current protection offered by the 10 -valent and 13 -valent pneumococcal conjugate vaccines were $33.3 \%$ and $41.6 \%$ respectively. Thus non-vaccine serotypes were featured prominently in our setting.

No conflict of interest

\title{
VIETNAM PNEUMOCOCCAL PROJECT: EVALUATION OF DIFFERENT INFANT VACCINATION SCHEDULES INCORPORATING PNEUMOCOCCAL VACCINATION
}

\author{
T.N. Huu ${ }^{1}$, T.M.P. Nguyen ${ }^{1}$, T.Q.H. Vu ${ }^{2}$, Y.U. Doan ${ }^{3}$, B. Temple ${ }^{4}$, K. Brightt ${ }^{4}$, T.T.D. Vo ${ }^{2}$, J.Y.R. Lai ${ }^{4}$, E.K. Mulholland ${ }^{4}$ \\ ${ }^{1}$ Control Disease Center, Pasteur Institute of Ho Chi Minh city, Ho Chi Minh, Vietnam; ${ }^{2}$ Immunology and Microbiology, Pasteur Insti- \\ tute of Ho Chi Minh city, Ho Chi Minh, Vietnam; ${ }^{3}$ Clinical Research Unit, Pasteur Institute of Ho Chi Minh city, Ho Chi Minh, Vietnam; ${ }^{4}$ Men- \\ zies School of Health Research, Charles Darwin University, Darwin, Australia
}

Background and Aims: Pneumonia is responsible for approximately 1.2 million deaths each year in children less than 5 years old, with the bulk of those occurring in Southeast Asia and sub-Saharan Africa. Pneumococcal vaccines can prevent vaccine-type pneumococcal pneumonia. However the data pertaining to the use of pneumococcal vaccines in Asia is scarce. This is a phase II/III, open-labelled randomized controlled clinical trial. The overall aim of the study is to address the outstanding questions that will guide the identification of a new schedule for routine immunization in Asia to support the most efficient use of pneumococcal conjugate vaccines.

This study will address two key questions regarding vaccine introduction into Asia:

1. What is the optimal schedule for provision of expanded programme of immunization (EPI) vaccines with the incorporation of Synflorix?

2. How do the responses to PCV vaccination with Synflorix or Prevnar-13 compare?

Methods: 1200 infants aged between 60 to 74 days old will be recruited from 2 study sites located in District 4 and District 7 of Ho Chi Minh City. Subjects will be randomized to one of six vaccination schedules.

Participants provide a series of blood samples for analysis of vaccine responses, and nasopharyngeal swabs for analysis of pneumococcal carriage.

Conclusion: This clinical trial will provide answers to the two key questions that have inhibited introduction of routine pneumococcal vaccination into this region. This trial will provide critical evidence based research to guide policy regarding inclusion of these important vaccines into EPI in Asian countries

No conflict of interest

ISPPD-0359

Controlling Pneumococcal Disease Around the Globe

\section{MATHEMATICAL MODELLING OF THE LONG-TERM POPULATION EFFECTS OF PNEUMOCOCCAL VACCINES ON CARRIAGE OF PNEUMOCOCCAL SEROTYPES IN KENYA}

\author{
J. Ojal' ${ }^{1}$, J.A.G. Scott ${ }^{1}$, L.L. Hammitt ${ }^{2}$, M. Nurhonen ${ }^{3}$, K. Auranen ${ }^{3}$ \\ ${ }^{1}$ Department of Epidemiology and Demography, KEMRI-Wellcome Trust Research Programme, Kilifi, Kenya; ${ }^{2}$ International Health, Johns Hop- \\ kins Bloomberg School of Public Health, Baltimore Maryland, USA; ${ }^{3}$ Department of Vaccination and Immune Protection, National Insti- \\ tute for Health and Welfare (THL), Helsinki, Finland
}

Background: The pneumococcal conjugate vaccines (PCVs) are a major advance in the control of pneumococcal disease but their major drawback is serotype replacement. Kenya introduced the ten-valent PCV in January 2011 with catch-up campaigns in children aged 1-5 years in one district. Since vaccination impact can vary across populations, we predicted the long-term effects of the vaccination programme in Kenya.

Methods: A dynamic compartmental model was fitted to local data. The model was then used to simulate the prevalence of vaccine-serotype (VT) and non-vaccine-serotype (NVT) Streptococcus pneumoniae carriage among Kenyan children over a ten-year period following PCV introduction. Non-vaccine serotypes were divided into two groups, strong and weak, based on the susceptibility to competition in the nasopharynx.

Results: The overall prevalence of $S$. pneumoniae is expected to reduce marginally from $43 \%$ to about $41 \%$ in the decade after introduction. The prevalence of VT carriage is projected to decline in all age groups over the decade, from $37 \%$ to $13 \%$ in infants, $6 \%$ to $2 \%$ in $20-49$ year olds, and $6 \%$ to $5 \%$ in those aged $50+$ years. The prevalence of NVT carriage is projected to rise from $47 \%$ to $78 \%$ in infants. 
Conclusion: The PCV immunisation programme is projected to reduce VT carriage substantially in infants with most of the reduction occurring in the first six years. Because complete serotype replacement carriage is predicted, trends in the post-vaccination incidence of pneumococcal disease depend on the average NVT case-to-carrier ratio as compared to the average VT case-to-carrier ratio.

No conflict of interest

ISPPD-0547

Controlling Pneumococcal Disease Around the Globe

PNEUMOCOCCAL SEROTYPES CAUSING CHILDHOOD INVASIVE PNEUMOCOCCAL DISEASE IN TURKEY, 2011-2012

\author{
M. Ceyhan ${ }^{1}$, N. Gurler ${ }^{2}$, Y. Ozsurekci ${ }^{1}$, E. Karadag Oncel ${ }^{1}$, M. Celik ${ }^{1}$, A. Ozkaya-Parlakay ${ }^{1}$, A. Aycan ${ }^{1}$, V. Gurbuz ${ }^{1}$
}

${ }^{1}$ Pediatric Infectious Diseases, Hacettepe University, Ankara, Turkey; ${ }^{2}$ Department of Clinical Microbiology, Istanbul University, Istanbul, Turkey

Background and Aims: Streptococcus pneumoniae (pneumococcus) is a major cause of severe pneumonia and other invasive diseases responsible for considerable morbidity and mortality in children across the world. In November 2008, PCV7 was included in the National Immunization Programme (NIP) in Turkey for children aged <2 years; and replaced with PCV13 in April 2011. In this study, we aimed to show the serotype distribution of pneumococcal strains causing invasive pneumococcal disease (IPD) in Turkish children in 2011-2012 period.

Method: The pneumococci were isolated from blood, cerebrospinal fluid and other body fluids of pediatric cases ( $<18$ years of age) with IPD and serotyping was performed using conventional methods.

Results: Pneumococci were isolated and serotyped in 67 samples. Coverage rates for PCV 7, PCV 10 and PCV 13 vaccines were $48 \%, 51 \%$ and $71 \%$ for $\leq 5$ years of age and $32 \%, 37 \%$ and $56 \%$ for $<18$ years of age, respectively. Serotype 3, 19A and 6A were the most frequent non-PCV 7 serotypes.

Conclusion: According to our data, PCV13 provides the highest serotype coverage rate among PCVs and would potentially be the most protective against childhood IPD in Turkey. We believe that ongoing IPD surveillance is important for appropriate vaccine recommendations.

No conflict of interest

\author{
ISPPD-0546 \\ Controlling Pneumococcal Disease Around the Globe
}

\title{
BACTERIAL ETIOLOGY AND PNEUMOCOCCAL SEROTYPES IN TURKISH CHILDREN WITH ACUTE OTITIS MEDIA
}

M. Ceyhan ${ }^{1}$, Y. Ozsurekci ${ }^{1}$, N. Gurler ${ }^{2}$, E. Karadag Oncel ${ }^{1}$, M. Celik ${ }^{1}$, A.E. Aycan ${ }^{1}$, V. Gurbuz ${ }^{1}$, U. Akyol ${ }^{3}$, O. Altuntas $^{3}$,

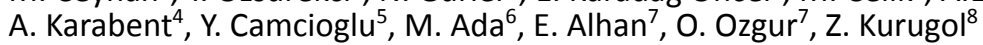

${ }^{1}$ Pediatric Infectious Diseases, Hacettepe University, Ankara, Turkey; ${ }^{2}$ Department of Clinical Microbiology, Istanbul University, Istanbul, Turkey; ${ }^{3}$ Department of Otorhinolaryngology, Hacettepe University, Ankara, Turkey; ${ }^{4}$ Pedaitrics, Cankiri State Hospital, Cankiri, Turkey; ${ }^{5}$ Pediatric Infectious Diseases, Istanbul University Faculty of Medicine, Istanbul, Turkey; ${ }^{6}$ Department of Otorhinolaryngology, Istanbul University Faculty of Medicine, Istanbul, Turkey; ${ }^{7}$ Pediatric Infectious Diseases, Cukurova University Faculty of Medicine, Adana, Turkey; ${ }^{8}$ Department of Pediatrics, Ege University Faculty of Medicine, Izmir, Turkey

Background: Acute otitis media (AOM) is one of the most common childhood diseases requiring antimicrobial prescription drugs in pre-school children. In this study, we sought to describe the bacterial etiology of pediatric cases of AOM in Turkey.

Materials and Methods: This prospective, multi-center, tympanocentesis-based epidemiological study was performed in children aged between 3 months and 6 years. All isolates were cultured and serogrouped by the Quellung reaction.

Results: During 2010-2012, 38 cases fulfilled the study inclusion criteria. Overall, $45 \%$ of samples were culture positive for bacterial pathogens Streptococcus pneumoniae (13.1\%) was the leading cause of bacterial AOM followed by Streptococcus pyogenes (10.5\%) and Haemophilus influenzae (7.9\%). Serotype-3 was detected in two of the samples, and serotypes 9V, 19, and 19A were isolated from one patient each. S. pneumoniae was detected in $36 \%$ (4/11) of otorrhea samples. All H.influenzae-positive samples were collected by tympanocentesis. All $H$. influenzae isolates were identified as non-typeable. The pneumococcal serotype coverage rates for PCV-7, PVC-10 and PCV-13 were $20 \%(1 / 5), 20 \%(1 / 5)$, and $80 \%(4 / 5)$, respectively. PHiD-CV (PCV-7 types plus 1, 5, and 7F) targets non-typeable $H$. influenzae, and 4 of $38(11 \%)$ of the pathogens causing episodes of AOM were also covered.

Conclusion: In Turkey, S. pneumoniae remains the most common pathogen in children with AOM. Both $S$. pneumoniae and non-typeable $H$. influenzae represent important targets for vaccination strategies to reduce AOM in children. Based on our results, conjugated pneumococcal vaccines may have potential impact to decrease the burden of AOM.

No conflict of interest 
ISPPD-0563

Controlling Pneumococcal Disease Around the Globe

\title{
EFFICACY OF THE 7-VALENT PNEUMOCOCCAL CONJUGATE VACCINE (PCV7) AGAINST ACUTE OTITIS MEDIA DUE TO SEROTYPE 6C IN THE FINOM TRIAL
}

\author{
A. Palmu' ${ }^{1}$, T. Kaijalainen², J. Jokinen ${ }^{3}$, T.M. Kilpi ${ }^{3}$ \\ ${ }^{1}$ Department of Vaccination and Immune Protection, National Institute for Health and Welfare, Tampere, Finland; ${ }^{2}$ Department of Vaccina- \\ tion and Immune Protection, National Institute for Health and Welfare, Oulu, Finland; ${ }^{3}$ Department of Vaccination and Immune Protection, \\ National Institute for Health and Welfare, Helsinki, Finland
}

A new pneumococcal serotype 6C was discovered in 2007, earlier typed as 6A. In the Finnish Otitis Media (FinOM) Vaccine Trial conducted in 1995-99, we reported (Eskola 2001) cross-protective serotype-specific vaccine efficacy (VE) of the PCV7 for 6A acute otitis media (AOM) as $57 \%(95 \% \mathrm{Cl} 24$ to 76). Now, we have re-typed all 6A isolates to evaluate the VE against 6C AOM. In the phase III randomized, double-blind controlled FinOM trial children received PCV7 or hepatitis B vaccine as control at 2, 4,6 and 12 months of age and were followed for AOM in specific study clinics. Myringotomy with middle ear fluid aspiration was performed in each case of AOM and the samples were cultured, Streptococcus pneumoniae were identified and serotyped using serological methods. Per protocol followup started 14 days after the third vaccination and ended at 24 months of age. Generalized Cox regression was used to estimate VE against 6A/6C AOM episodes. 831 children were enrolled both in the PCV7 and control arms. During the per protocol follow-up period, ten AOM episodes due to serotype $6 \mathrm{C}$ were found, 5 in both treatment groups (VE $-1,95 \% \mathrm{Cl}-248$ to 71$)$. The updated VE estimate for serotype $6 \mathrm{~A} A O M$ was $65 \%(95 \% \mathrm{Cl} 31$ to 82$)$.

In the control group, serotype $6 \mathrm{C}$ caused $11 \%$ of the episodes originally serotyped as $6 \mathrm{~A}$. PCV7 vaccine offered no protection against $6 \mathrm{C} \mathrm{AOM}$, but had excellent efficacy against 6A AOM. There are, however, recent data that PCV13, which includes $6 \mathrm{~A}$ antigen, offers cross-protection against $6 \mathrm{C}$.

Conflict of interest

ISPPD-0463

Controlling Pneumococcal Disease Around the Globe

\begin{abstract}
INDIRECT IMPACT OF PNEUMOCOCCAL HAEMOPHILUS INFLUENZAE PROTEIN-D CONJUGATE VACCINE (PHIDCV10) ON SUSPECTED NON-CONFIRMED INVASIVE PNEUMOCOCCAL DISEASE (IPD) IN CLUSTER-RANDOMIZED TRIAL
\end{abstract}

A.A. Palmu ${ }^{1}$, J. Jokinen ${ }^{2}$, E. Ruokokoski², H. Nieminen ${ }^{1}$, T. Puumalainen ${ }^{3}$, M. Moreira ${ }^{4}$, L. Schuerman ${ }^{4}$, D. Borys ${ }^{4}$, T.M. Kilpi

${ }^{1}$ Department of Vaccination and Immune Protection, National Institute for Health and Welfare, Tampere, Finland; ${ }^{2}$ Department of Vaccination and Immune Protection, National Institute for Health and Welfare, Helsinki, Finland; ${ }^{3}$ Vaccines, GlaxoSmithKline, Espoo, Finland

${ }^{4}$ Vaccines, GlaxoSmithKline, Wavre, Belgium

Background: In the nation-wide double-blind cluster-randomized Finnish Invasive Pneumococcal disease (FinIP) trial we assessed the indirect impact of 10-valent pneumococcal Haemophilus influenzae protein D conjugate vaccine (PHiD-CV10) (GlaxoSmithKline Vaccines) on suspected IPD using hospital diagnoses.

Methods: Children $<19$ months received PHiD-CV10 in 48 clusters or hepatitis B/A vaccine as control in 24 clusters according to infant $3+1$ or $2+1$ schedules or catch-up schedules. Hospitals' in/outpatient discharge reports with ICD-10 diagnoses compatible with IPD and unspecified sepsis and/or infection were collected from national Care Register for the whole population. Cases of IPD detected by culture or DNA/RNA detection were excluded. Annual incidences in the non-vaccinated population aged $>5$ years were compared between the PHiD-CV10 and control clusters. National vaccination programme for 3-month-old infants without catch-up started in Sep-2010.

Results: Altogether, $>41,000$ children were enrolled from May-2009 to Oct-2010. Trial vaccination coverage varied from 29 to $61 \%$ in the PHiD-CV10 clusters. In 2011, 104 suspected, non-confirmed IPD episodes (ICD-10 A40.3/ $\mathrm{B} 95.3 / \mathrm{G} 00.1 / \mathrm{M} 00.1$ ) were detected in the unvaccinated population aged $>5$ years. The table shows the preliminary results.

Table: Hospital-diagnosed suspected non-confirmed IPD in unvaccinated population >5 years in 2011

\begin{tabular}{|c|c|c|c|c|}
\hline \multirow[t]{2}{*}{ Endpoint } & \multicolumn{2}{|c|}{ Rate/100000 person-years } & \multirow[t]{2}{*}{ VE(\%) } & \multirow[t]{2}{*}{$95 \% \mathrm{Cl}$} \\
\hline & $\begin{array}{l}\text { PHiD-CV10 clus- } \\
\text { ters }\end{array}$ & Control clusters & & \\
\hline Suspected, non-confirmed IPD, 3+1 clusters & 2,9 & 3,4 & 12 & -37 to 43 \\
\hline Suspected, non-confirmed IPD, 2+1 clusters & 1,9 & 3,4 & 46 & 11 to 67 \\
\hline $\begin{array}{l}\text { Suspected, non-confirmed IPD, 3+1 and 2+1 } \\
\text { clusters combined }\end{array}$ & 2,4 & 3,4 & 29 & -6 to 35 \\
\hline
\end{tabular}

Conclusion: This is the first report presenting the indirect impact of PCV on suspected non-confirmed IPD. Indirect impact of the trial vaccination was seen despite lower than optimal vaccination coverage.

Conflict of interest 
ISPPD-0384

Controlling Pneumococcal Disease Around the Globe

\title{
EFFECTIVENESS OF THE PNEUMOCOCCAL HAEMOPHILUS INFLUENZAE PROTEIN D CONJUGATE VACCINE (PHID-
} CV10) AGAINST OUTPATIENT ANTIMICROBIAL PRESCRIPTIONS - FINIP TRIAL EXTENDED FOLLOW-UP

\author{
A.A. Palmu ${ }^{1}$, J. Jokinen ${ }^{2}$, H. Nieminen ${ }^{1}$, H. Rinta-Kokko ${ }^{2}$, E. Ruokokoski ${ }^{2}$, T. Puumalainen ${ }^{3}$, D. Borys ${ }^{4}$, M. Moreira ${ }^{4}$, \\ L. Schuerman ${ }^{4}$, T.M. Kilpi ${ }^{2}$ \\ ${ }^{1}$ Department of Vaccination and Immune Protection, National Institute for Health and Welfare, Tampere, Finland; ${ }^{2}$ Department of Vaccina- \\ tion and Immune Protection, National Institute for Health and Welfare, Helsinki, Finland; ${ }^{3}$ Vaccines, GlaxoSmithKline, Espoo, Finland \\ ${ }^{4}$ Vaccines, GlaxoSmithKline, Wavre, Belgium
}

Background: Pneumococcal conjugate vaccines (PCV) have reduced respiratory infections, although modestly. We showed $8 \%(95 \% \mathrm{Cl} 1-14)$ reduction on antimicrobial consumption during the blinded follow-up and have now evaluated the longer-term impact of the 10-valent PHiD-CV (GlaxoSmithKline Vaccines).

Methods: In this nationwide phase III/IV cluster-randomized, double-blind trial, children <19 months received PHiD-CV10 in two thirds of clusters $(n=52)$ or hepatitis B or A vaccine as control in 26 clusters according to $3+1$ or $2+1$ schedules (infants $<7$ months) or catch-up schedules (children 7-18 months). The main objective of this analysis was to assess long-term vaccine effectiveness (VE) against outpatient prescriptions for antimicrobials recommended for acute otitis media (AOM) by the national treatment guidelines. The blinded follow-up ended in Dec 2011 after which we extended the register follow-up for year 2012. Outcome data i.e. all purchased prescriptions, were obtained from the benefits register of the Social Insurance Institution.

Results: Over 47,000 children were enrolled from 2009 through 2010. In 30527 infants <7 months of age at enrolment, >30000 outpatient antimicrobial prescriptions recommended for AOM were reported during year 2012 with incidence of 0.99 per person-year in the control clusters. The preliminary VE estimate for the 2012 follow-up was $1 \%(95 \% \mathrm{Cl} ;-7$ to $9 \%)$ for combined PHiD-CV10-3+1/2+1 infant schedules and $4 \%$ (-4 to $12 \%)$ for the catch-up schedules.

Conclusion: The extended follow-up shows no long-term impact on antimicrobial use in children after two to three years of age. National PCV vaccination programme started in Sep 2010 and its indirect effect may have diminished the impact during the extended follow-up.

Conflict of interest

ISPPD-0387

Controlling Pneumococcal Disease Around the Globe

\section{EPIDEMIOLOGICAL AND CLINICAL CHARACTERISTICS OF INVASIVE PNEUMOCOCCAL DISEASES IN PILSEN REGION (CZECH REPUBLIC)}

P. Pazdiora ${ }^{1}$, J. Kozakova²

${ }^{1}$ Epidemiology, Medical Faculty of Charles University, Pilsen, Czech Republic; ${ }^{2}$ National Reference Laboratory for Streptococcal Infections, National Institute of Public Health, Prague, Czech Republic

Background and Aims: Due to changing epidemiological situation of invasive pneumococcal diseases (IPD) in the association with the vaccination, even the regional data from different age-groups are important. Methods: Pneumococcal meningitis reported in 1997-2007 was analysed retrospectively, all IPD in 2008-2012 (2013) have been monitored prospectively.

Results: 57 cases of pneumococcal meningitis (morbidity rate $0.7 / 100,000$ inhabitants per year) were reported in 1997-2012. The highest specific morbidity rate was found out in children up to 1 year of age $(7.4 / 100,000)$. After the initiating of the statewide surveillance of all IPD, 145 diseases ( 42 cases of septicaemia, 18 cases of meningitis, 85 cases of pneumonia) were reported within January 2008 - December 2012. The highest specific morbidity rate was found out in 75-year-old persons and older (17.1/100,000), 17 diseases out of the total were lethal, all the deaths in persons over 45. The most frequent risk factor for the occurrence IPD was age over 65 years and smoking. Serotypisation was carried out in 140 strains of pneumococcus, serotype $3(22 x)$ being proved most frequently. The changes of morbidity and serotypes in 2013 are discussed.

Conclusion: It is evident from the results that in the Pilsen Region IPD are currently important mainly for adults both by their frequency and seriousness. The routine vaccination of children, which started in the Czech Republic in 2010, can influence the incidence in older persons only partially.The priority must be the support the vaccination among adults, too. Some variations in the occurrence of individual serotypes of pneumococcus can be expected.

No conflict of interest 
ISPPD-0526

Controlling Pneumococcal Disease Around the Globe

\title{
IMPACT OF PNEUMOCOCCAL VACCINE ON INVASIVE PNEUMOCOCCAL DISEASE AMONG YOUNG CHILDREN IN THE MID-WEST REGION OF IRELAND
}

\author{
R.K. Philip ${ }^{1}$, N. OConnell ${ }^{2}$, J. Powell ${ }^{2}$, R. Fitzgerald ${ }^{3}$ \\ ${ }^{1}$ Paediatrics, University Hospital Limerick, Limerick, Ireland; ${ }^{2}$ Microbiology, University Hospital Limerick, Limerick, Ireland; ${ }^{3}$ Population Health, \\ Health Service Executive, Limerick, Ireland
}

Background and Aims: Pneumococcal vaccine (PCV 7) was included in the National immunisation schedule of Ireland in September 2008. Significant reduction in morbidity and case specific mortality was expected. We aimed to, 1. establish the reduction in hospital admission for children due to invasive pneumococcal disease (IPD) since the introduction of pneumococcal vaccine in the Mid-West region of Ireland, 2. to compare with the corresponding figures for the whole country, and 3. to determine the added clinical value of the extended spectrum vaccine (PCV13) that commenced in 2010.

Methods: All admissions below five years of age registered on the hospital inpatient enquiry system (HIPE) with the confirmed diagnosis of IPD or recognised in the computerised laboratory data as a positive Streptococcus pneumoniae culture/PCR from blood, pleural effusion or cerebrospinal fluid were tabulated for 5 years before and 5 years after the HPV introduction to the National immunisation schedule. Number of cases against the birth cohort of the region (5000/year) was calculated.

Results: There was reduction of $90 \%$ and $72 \%$ respectively for IPD below two years and two to five years age group after introduction of PCV. Our observations are similar to the National published figures. Since the introduction of HPV13 in late 2010 further reduction in hospital admission and incidence of complicated pneumonia was observed. Current PCV uptake in the region is $90 \%$.

Conclusion: In a developed health care setting, Immunisation with PCV offer significant reduction in hospital admission due to invasive pneumococcal disease.

No conflict of interest

\section{BY CLINICAL PRESENTATION INCIDENCE OF INVASIVE PNEUMOCOCCAL DISEASE AFTER WITHDRAWAL OF PCV13 FROM THE PEDIATRIC UNIVERSAL VACCINATION CALENDAR IN MADRID}

J. Picazo ${ }^{1}$, J. Ruiz-Contreras ${ }^{2}$, J. Casado-Flores ${ }^{3}$, S. Negreira² ${ }^{2}$, F. Baquero ${ }^{4}$, T. Hernandez-Sampelayo ${ }^{5}$, E. Otheo ${ }^{6}$, C. Mendez ${ }^{7}$, on behalf of the HERACLES study group ${ }^{8}$

${ }^{1}$ Microbiology, Hospital Clínico San Carlos, Madrid, Spain; ${ }^{2}$ Pediatrics, Hospital Doce de Octubre, Madrid, Spain; ${ }^{3}$ Pediatric ICU, Hospital Niño Jesus, Madrid, Spain; ${ }^{4}$ Pediatrics, Hospital La Paz, Madrid, Spain; ${ }^{5}$ Pediatrics, Hospital Gregorio Marañon and CIBER of Respiratory Diseases, Madrid, Spain; ${ }^{6}$ Pediatrics, Hospital Ramon y Cajal, Madrid, Spain; ${ }^{7}$ Medical, Pfizer, Madrid, Spain; ${ }^{8}$ Madrid, Spain

Background and Aims: Surveillances at clinical presentation level are essential to explore effects of changes in vaccination strategies. PCV7 was introduced for universal vaccination of children $<24$ months in Madrid $(6$ million inhabitants) in 2006 and replaced by PCV13 (with catch-up for children 18-24 months) in May 2010. In May 2012, PCV13 was withdrawn from the universal vaccination calendar, being available in private market. We compared incidence rates (IR) of clinical presentations of invasive pneumococcal disease (IPD) in children <15 years requiring hospitalization in May 2007-April 2010 (PCV7 period) vs. May 2012-April 2013.

Methods: A prospective, laboratory-confirmed (culture and/or PCR) surveillance of all hospitalized IPDs in Madrid was performed. IR (no. cases/100,000 inhabitants) was calculated using data from the Spanish Instituto Nacional de Estadistica. The estimated vaccination coverage in 2012-3 (using data from IMS) was 70\% for PCV13 (schedule) in children $<12 \mathrm{mo}(3+1)$ and $95 \%$ in those $12-59$ mo $(2+1+$ catch-up), and $50 \%$ for PCV7 in children $\geq 60$ mo- $<15$ y $(3+1)$. Comparisons of IR were performed (EPIDAT version 3.1).

Results: The Table shows per-period and per-clinical presentations, IRs and \% reduction in 2012-3.

\begin{tabular}{|l|l|l|l|l|l|l|}
\hline & $2007-2010$ & \multicolumn{3}{l|}{$2012-2013$} \\
\hline Population & 2920346 & \multicolumn{3}{l|}{1008491} \\
\hline & $\mathrm{IR}$ & & $\mathrm{IR}$ & $\mathrm{p}^{*}$ & $\%$ Reduction \\
\hline Bacteremic pneumonia & 5.51 & & 0.69 & $<0.001$ & 87.40 \\
\hline Parapneumonic pneumococcal empyema & 5.72 & & 2.18 & $<0.001$ & 61.85 \\
\hline Primary bacteremia & 2.05 & & 1.09 & 0.068 & 46.79 \\
\hline Meningitis & 2.16 & & 0.59 & 0.002 & 72.46 \\
\hline Other & 1.64 & & 0.79 & 0.072 & 51.63 \\
\hline & & & *vs. 2007-2010 \\
\hline
\end{tabular}


Conclusion: IRs of all IPD clinical presentations were markedly reduced in 2012-3 (with statistically significant reductions for respiratory presentations and meningitis) regardless withdrawal of pneumococcal vaccines from the vaccination calendar (high private uptake of PCV13).

Document not received

ISPPD-0189

Controlling Pneumococcal Disease Around the Globe

\section{SUPPURATIVE OTITIS MEDIA BY STREPTOCOCCUS PNEUMONIAE AND/OR HAEMOPHILUS INFLUENZAE: NASOPHARYNGEAL CARRIAGE, ISOLATED SEROTYPES AND ANTIMICROBIAL SUSCEPTIBILITY IN BARCELONA (HERMES STUDY)}

J. Picazo ${ }^{1}$, R. Cilveti ${ }^{2}$, M. Olmo ${ }^{2}$, J. Perez-Jove ${ }^{3}$, J.L. Arimany ${ }^{4}$, E. Mora ${ }^{5}$, T. Perez-Porcuna ${ }^{6}$, I. Aguilar ${ }^{7}$, A. Alonso ${ }^{8}$, F. Molina ${ }^{9}$, C. Mendez ${ }^{10}$

${ }^{1}$ Microbiology, Hospital Clínico San Carlos, Madrid, Spain; ${ }^{2}$ Pediatrics, Hospital Mutua de Terrassa, Barcelona, Spain; ${ }^{3}$ Microbiology, Catlab, Barcelona, Spain; ${ }^{4}$ Pediatrics, Hospital General de Cataluña, Barcelona, Spain; ${ }^{5}$ Pediatrics, Hospital Clinico San Carlos, Madrid, Spain; ${ }^{6}$ Pediatrics, CAP Valdoreix, Barcelona, Spain; ${ }^{7}$ Pediatrics, CAP San Cugat, Barcelona, Spain; ${ }^{8}$ Pediatrics, CAP Rambla, Barcelona, Spain; ${ }^{9}$ Pediatrics, CAP Terrassa Sud, Barcelona, Spain; ${ }^{10}$ Medical, Pfizer, Madrid, Spain

Background and Aims: Serotypes/susceptibility of Streptococcus pneumoniae (Sp) and Haemophilus influenzae (Hi) isolates from otical/nasopharyngeal samples in children were explored in a well-defined area $(400,000$ inhabitants) of Barcelona (private market coverage: $50 \%$ ).

Methods: Nasopharyngeal and middle ear swabs from 238 children ( $2 \mathrm{mo}-\leq 8 \mathrm{y}$ ) presenting otitis with spontaneous suppuration in a multicenter prospective study were sent to central laboratory. PCR detection ( $\mathrm{Sp}, \mathrm{Hi}$ ) was performed in culture-negative ear samples.

Results: $87 \mathrm{Sp}, 97 \mathrm{Hi}$ were identified in otical samples (both concomitant in 39). In nasopharynx, $112 \mathrm{Sp}, 78 \mathrm{Hi}$ were recovered. Non-typeable (by PCR) Sp: 39 (otical), 20 (nasopharyngeal). Tables show by origin PCV13 and non-PCV13 serotypes.

\begin{tabular}{|l|l|l|l|l|}
\hline PCV13 & \multicolumn{2}{|l}{ Otical } & Nasopharyngeal \\
\hline & $\mathrm{Sp}$ & $\mathrm{Sp}+\mathrm{Hi}$ & Total Sp & $\mathrm{Sp}$ \\
\hline $9 \mathrm{~V} / 14 / 18 \mathrm{C} / 19 \mathrm{~F} / 23 \mathrm{~F}$ & $1 / 1 / 1 / 2 / 0$ & $0 / 0 / 0 / 2 / 0$ & $1 / 1 / 1 / 4 / 0$ & $1 / 1 / 2 / 6 / 1$ \\
\hline 1 & 1 & 0 & 1 & 1 \\
\hline 3 & 3 & 3 & 6 & 3 \\
\hline 5 & 0 & 1 & 1 & 0 \\
\hline 6 & 1 & 1 & 2 & 4 \\
\hline $7 \mathrm{~F}$ & 2 & 1 & 3 & 1 \\
\hline $19 \mathrm{~A}$ & 9 & 2 & 11 & 12 \\
\hline Total & 21 & 10 & 31 & 32 \\
\hline
\end{tabular}

\begin{tabular}{|l|l|l|l|l|}
\hline Non-PCV13 & \multicolumn{2}{|l|}{ Otical } & Nasopharyngeal \\
\hline & Sp & Sp +Hi & Total Sp & Sp \\
\hline $11 \mathrm{~A}$ & 1 & 1 & 2 & 9 \\
\hline $24 \mathrm{~A}$ & 1 & 1 & 2 & 6 \\
\hline $23 \mathrm{~B}$ & 1 & 0 & 1 & 5 \\
\hline 21 & 1 & 1 & 0 & 4 \\
\hline $15 \mathrm{~A}$ & 2 & 0 & 2 & 4 \\
\hline $22 \mathrm{~F}$ & 1 & 0 & 1 & 4 \\
\hline Other & 7 & 0 & 9 & 28 \\
\hline Total & 14 & 3 & 17 & 60 \\
\hline
\end{tabular}

Oral penicillin/erythromycin non-susceptibility: 39.7\%/37.6\% for $\mathrm{Sp}(\mathrm{n}=141)$ and $64.7 \% / 64.7 \%$ for $19 \mathrm{~A}$ $(n=17)$ isolates. Only $3(2.2 \%)$ of $137 \mathrm{Hi}$ isolates were betalactamase-positive and $5(3.6 \%)$ ampicillin-resistant betalactamase-negative.

Conclusion: Sp nasopharyngeal carriage was high (47\%). PCV13 serotypes caused $35.6 \%$ otitis by Sp ( $\pm \mathrm{Hi}$ ). Nonsusceptibility was high (especially serotype 19A).

Conflict of interest 
ISPPD-0089

Controlling Pneumococcal Disease Around the Globe

\section{CHANGES IN INCIDENCE OF SEROTYPE-SPECIFIC INVASIVE PNEUMOCOCCAL DISEASE (IPD) AFTER WITHDRAWAL OF PCV13 FROM THE PEDIATRIC VACCINATION CALENDAR IN MADRID}

J. Picazo ${ }^{1}$, J. Ruiz-Contreras ${ }^{2}$, J. Casado-Flores ${ }^{3}$, S. Negreira² ${ }^{2}$ F. Baquero ${ }^{4}$, T. Hernandez-Sampelayo ${ }^{5}$, E. Otheo ${ }^{6}$, C. Mendez ${ }^{7}$, on behalf the HERACLES study group ${ }^{8}$

${ }^{1}$ Microbiology, Hospital Clínico San Carlos, Madrid, Spain; ${ }^{2}$ Pediatrics, Hospital Doce de Octubre, Madrid, Spain; ${ }^{3}$ Pediatric ICU, Hospital Niño Jesus, Madrid, Spain; ${ }^{4}$ Pediatrics, Hospital La Paz, Madrid, Spain; ${ }^{5}$ Pediatrics, Hospital Gregorio Marañon and CIBER Respiratory Diseases, Madrid, Spain; ${ }^{6}$ Pediatrics, Hospital Ramon y Cajal, Madrid, Spain; ${ }^{7}$ Medical, Pfizer, Madrid, Spain; ${ }^{8}$ Madrid, Spain

Background and Aims: Benefits of replacing PCV7 (November 2006-April 2010) by PCV13 (May 2010-May 2012) in the paediatric vaccination calendar in Madrid has been previously reported. From May 2012 PCV13 is only available in private market. We compared incidence rates (IR) of serotype-specific IPD in children $<15$ years requiring hospitalization in May 2007-April 2010 (PCV7 period) vs. May 2012-April 2013.

Methods: A prospective, laboratory-confirmed (culture and/or PCR) surveillance of all hospitalized IPDs in Madrid was performed. All isolates (for serotyping) and culture-negative pleural/cerebrospinal fluids (for PCR detection) were sent to central laboratory. IR (no. cases/100,000 inhabitants) was calculated using data from the Spanish Instituto Nacional de Estadistica. The estimated vaccination coverage in 2012-2013 (using data from Intercontinental Marketing Services) was 70\% for PCV13 (schedule) in children $<12 \mathrm{mo}(3+1)$ and $95 \%$ in those $12-59$ mo $(2+1+$ catchup), and $50 \%$ for PCV7 in children $\geq 60$ mo-<15 y (3+1). Comparisons of IR were performed (EPIDAT version 3.1). Results: Table shows per-period (population) and serotype, IRs and \% reduction in 2012-3.

\begin{tabular}{|c|c|c|c|c|}
\hline & 2007-2010 (2920346) & \multicolumn{3}{|c|}{ 2012-2013 (1008491) } \\
\hline IPD by serotype & IR & IR & $p^{*}$ & \%IR reduction \\
\hline 1 & 4.79 & 0.89 & $<0.001$ & 81.37 \\
\hline 3 & 0.89 & 0.79 & 0.908 & 10.87 \\
\hline $7 F$ & 1.44 & 0.20 & 0.004 & 86.23 \\
\hline $19 \mathrm{~A}$ & 3.77 & 0.20 & $<0.001$ & 94.74 \\
\hline PCV7 & 0.72 & 0.10 & 0.025 & 86.10 \\
\hline Total PCV13 & 13.49 & 2.18 & $<0.001$ & 83.84 \\
\hline \multirow[t]{2}{*}{ Non-PCV13 } & 3.60 & 3.17 & 0.602 & 11.94 \\
\hline & & \multicolumn{3}{|c|}{ *vs. 2007-2010 } \\
\hline
\end{tabular}

No cases by serotypes 5 and 6A were found in 2012-3.

Conclusion: IR of IPD by PCV13 serotypes significantly decreased without increases in IRs of IPDs by non-PCV13 serotypes regardless withdrawal of pneumococcal vaccines from the vaccination calendar but with a high private uptake of PCV13.

Conflict of interest

ISPPD-0087

Controlling Pneumococcal Disease Around the Globe

\section{BY AGE GROUP INCIDENCE OF INVASIVE PNEUMOCOCCAL DISEASE AFTER WITHDRAWAL OF PCV13 FROM THE PEDIATRIC UNIVERSAL VACCINATION CALENDAR IN MADRID}

J. Picazo ${ }^{1}$, J. Ruiz-Contreras ${ }^{2}$, J. Casado-Flores ${ }^{3}$, S. Negreira² ${ }^{2}$ F. Baquero ${ }^{4}$, T. Hernandez-Sampelayo ${ }^{5}$, E. Otheo ${ }^{6}$, C. Mendez ${ }^{7}$, on behalf of the HERACLES study group ${ }^{8}$

${ }^{1}$ Microbiology, Hospital Clínico San Carlos, Madrid, Spain; ${ }^{2}$ Pediatrics, Hospital Doce de Octubre, Madrid, Spain; ${ }^{3}$ Pediatric ICU, Hospital Niño Jesus, Madrid, Spain; ${ }^{4}$ Pediatrics, Hospital La Paz, Madrid, Spain; ${ }^{5}$ Pediatrics, Hospital Gregorio Marañon, Madrid, Spain; ${ }^{6}$ Pediatrics, Hospital Ramon y Cajal, Madrid, Spain; ${ }^{7}$ Medical, Pfizer, Madrid, Spain; ${ }^{8}$ Madrid, Spain

Background and Aims: In 2006 PCV7 was introduced for universal vaccination of children <24 months in Madrid (6 million inhabitants) and on May 2010 PCV13 (with catch-up for children 18-24 months) replaced PCV7. In May 2012, PCV13 was withdrawn from the universal vaccination calendar, being available in private market. We compared incidence rates (IR) of invasive pneumococcal disease (IPD) in children < 15 years requiring hospitalization in May 2007-April 2010 (PCV7 period) vs. May 2012-April 2013.

Methods: A prospective, laboratory-confirmed (culture and/or PCR) surveillance of all hospitalized IPDs was performed. All isolates (for serotyping) and culture-negative pleural/cerebrospinal fluids (for PCR detection) were sent to a central laboratory. IR (no. cases/100,000 inhabitants) was calculated using data from the Spanish Instituto Nacional de Estadistica. PCV13 vaccination in 2012-2013 was estimated using data from Intercontinental Marketing Services (IMS). Comparisons of IR were performed (EPIDAT version 3.1). 
Results:

Table 1: Estimated PCV7 and PCV13 coverages (\%) in 2012-2013.

\begin{tabular}{|l|l|l|}
\hline Age Group & $\%$ PCV7 (schedule) & \%PCV13 (schedule) \\
\hline$<12$ mo & - & $70(3+1)$ \\
\hline $12-59$ mo & - & $95(2+1)$ \\
\hline$\geq 60 \mathrm{mo}-<15$ y & $50(3+1)$ & - \\
\hline
\end{tabular}

Table 2: Population, IRs and IR reduction in 2012-2013 vs. 2007-2010.

\begin{tabular}{|l|l|l|l|l|l|}
\hline & \multicolumn{2}{|l|}{$\mathbf{2 0 0 7 - 2 0 1 0}$} & 2012-2013 \\
\hline Age group & Population & IR & Population & IR & \% IR reduction \\
\hline$<12$ mo & 224692 & 47.62 & 68049 & $11.76^{*}$ & 75.31 \\
\hline $12-59$ mo & 872911 & 31.85 & 292371 & $11.63^{*}$ & 63.49 \\
\hline$\geq 60$ mo-<15 y & 1822743 & 6.25 & 648071 & $1.85^{*}$ & 70.37 \\
\hline Total & 2920346 & 17.09 & 1008491 & $5.35^{*}$ & 68.66 \\
\hline
\end{tabular}

Conclusion: Regardless PCV13 withdrawal from the vaccination calendar, the estimated coverage was high (70\%) and IR of IPD was significantly reduced in all age groups.

Conflict of interest

ISPPD-0017

Controlling Pneumococcal Disease Around the Globe

\section{CLINICAL AND MICROBIOLOGICAL PROFILE OF INVASIVE PNEUMOCOCCAL DISEASE IN UNDER FIVE CHILDREN: AN INSTITUTION BASED STUDY}

J. Poroor ${ }^{1}$, A. Vijayan ${ }^{2}$, A. Dev ${ }^{3}$, S. Sankaran ${ }^{1}$, S. Tevaruparambil ${ }^{1}$, J. Kuruvilla ${ }^{1}$

${ }^{1}$ Pediatrics, Pushpagiri Institute of Medical Sciences and Research Centre, Tiruvalla, India; ${ }^{2}$ Microbiology, Pushpagiri Institute of Medical Sciences and Research Centre, Tiruvalla, India; ${ }^{3}$ Research, Pushpagiri Institute of Medical Sciences and Research Centre, Tiruvalla, India

Objective: Identify the clinical and microbiological characteristics of invasive pneumococcal disease among children aged 1 month to 5 years

Methodology: Conducted in Dept of Pediatrics, Pushpagiri Institute, Tiruvalla, Kerala from Jan 2011-June 2012 as prospective observational study. Children 1 month- 5 years with fever included and sterile sample collection done - blood, cerebrospinal fluid (CSF) or pleural fluid after consent. Bactec bottles used for blood and sterile bottles for others. Sheep blood agar used for plating. The samples of positive cultures were sent to Dept of Microbiology, CMC Vellore, from where batches of isolates were sent to the Central Lab (Statens Serum Institute, Copenhagen, Denmark) for susceptibility testing/ serotyping. Sensitivity done for penicillin (Pen), chloramphenicol (Chl), erythromycin (Ery), cotrimoxazole (SXT) and cefotaxim (Cef). Statistical tool-Fisher's Exact Test.

Results: 138 recruited and 94 cases included. 6 blood culture positives. Strain pattern, resistance and clinical picture shown in tables 1 and2.

Conclusion:

The strains identified - 19F, 19A, 23F and 14 (2).

$3 / 5$ were erythromycin resistant, $3 / 5$ cotrimoxazole resistant, $2 / 5$ chloramphenicol resistant and $1 / 5$ penicillin resistant.

- The predictive value of having pneumococcal sepsis increases if fever $>/=101^{\circ} \mathrm{F}$ and leukocytosis $>15000$ are taken together

Acknowledgement: ASIP study for the laboratory services

Table 1:

\begin{tabular}{|l|l|l|l|l|l|l|l|}
\hline Strain & Age & Diagnosis & Pen & Chl & Ery & SXT & Cef \\
\hline $23 \mathrm{~F}$ & 3 & Bacteremia & S & R & R & R & S \\
\hline $19 \mathrm{~F}$ & 3 & Bacteremia & S & S & R & S & S \\
\hline 14 & 1 & Bacteremia & S & S & R & R & S \\
\hline $19 \mathrm{~A}$ & 1 & Pneumonia & S & S & S & S & S \\
\hline 14 & 2 & Meningitis & R & R & R & R & S \\
\hline
\end{tabular}


Table 2:

\begin{tabular}{|l|l|l|l|}
\hline Parameters & No. & Pneumococcal sepsis & $p$ value \\
\hline TC $>15000$ (> 60\%poly)+ fever $>1010 \mathrm{~F}$ & 17 & $6(35.29 \%)$ & 0.00001 \\
\hline
\end{tabular}

Conflict of interest

ISPPD-0230

Controlling Pneumococcal Disease Around the Globe

\title{
A CRUCIAL TOOL IN PCV13 IMPACT MONITORING: PROSPECTIVE, ACTIVE SURVEILLANCE OF COMMUNITY ACQUIRED PNEUMONIA AMONG ADULTS, APULIA, ITALY, 2013-2014
}

\footnotetext{
D. Martinelli ${ }^{1}$, B. Pedalino ${ }^{1}$, V. Cozza ${ }^{1}$, F. Fortunato ${ }^{1}$, M.G. Cappelli ${ }^{2}$, P. Ciavarella ${ }^{1}$, A. Sallustio ${ }^{2}$, M. Chironna ${ }^{2}$, R. Prato ${ }^{1}$

${ }^{1}$ Settore di Igiene, University of Foggia, Foggia, Italy; ${ }^{2}$ University of Bari, Department of Biomedical Sciences and Human Oncology University of Bari, Bari, Italy
}

Background and Aims: Worldwide, Streptococcus pneumoniae is the most commonly identifiable cause of Community Acquired Pneumonia (CAP) resulting in hospitalizations and deaths in high-risk adults and elderly. Incidence varies between 2-40 cases/1,000 elderly person-years, case fatality ratio between 7-35\% in Europe/US. Little is known in Italy on the burden of this disease. Since 2000, 23-valent pneumococcal vaccine (PPV23) was offered in Apulia to individuals aged $>64$ years. Since 2011, 13-valent pneumococcal conjugate vaccine (PCV13) has been introduced targeting the same population. Overall (PPV23/PCV13) vaccination coverage was 10\% (August 2013). We described the epidemiology of CAP in adults in Apulia.

Methods: We implemented a two-year active sentinel surveillance system involving thirty physicians (general practitioners (GPs)/pneumologists) to identify patients with CAP signs and/or symptoms and collect: clinical information, vaccination history, nasopharyngeal swabs, sputum and/or blood specimens. Specimens were tested for S. pneumoniae identification and PCR serotyping. We analysed data using Stata/MP 11.2.

Results: In January-September 2013, 76 CAP were identified. Median age was 79 years (range 64-95); 60.5\% were males; vaccination history was available for $61(80 \%)$ patients. Physicians collected 182 specimens. We identified $29(29 / 76 ; 38 \%)$ CAP positive (in either blood or sputum) for S. pneumoniae. Mostly isolated serotypes were: $23 \mathrm{~F}$ (8/29), 14 (4/29), 3 (3/29), 4 (3/29), 12F (2/29), 9V (2/29). Serotyping was not possible for three sputum.

Conclusion: We are lightening the regional lack of longitudinal data on S. pneumoniae CAP. Most cases were due to vaccine-contained serotypes suggesting how paramount PCV13 vaccination uptake is to benefit of a substantial vaccine impact.

Conflict of interest

ISPPD-0229

Controlling Pneumococcal Disease Around the Globe PRELIMINARY PCV13 EFFECTIVENESS ESTIMATES IN ADULTS: A TEST-NEGATIVE DESIGN USING SURVEILLANCE OF
COMMUNITY ACQUIRED PNEUMONIA, APULIA, ITALY, JANUARY-SEPTEMBER 2013

\author{
B. Pedalino ${ }^{1}$, D. Martinelli ${ }^{1}$, M.G. Cappelli ${ }^{2}$, P. Ciavarella ${ }^{1}$, R. Russo ${ }^{1}$, F. Fortunato ${ }^{1}$, R. Prato ${ }^{1}$ \\ ${ }^{1}$ settore di Igiene, University of Foggia, Foggia, Italy; ${ }^{2}$ Department of Biomedical Sciences and Human Oncology, Department of Biomedical Sci- \\ ences and Human Oncology University of Bari, Bari, Italy
}

Background and Aims: Streptococcus pneumoniae is the most commonly identifiable cause of community acquired pneumonia (CAP) resulting in hospitalization and death in the elderly worldwide. Since 2000, 23-valent pneumococcal vaccine (PPV23) was offered in Apulia to individuals aged >64 years. Since 2011, 13-valent pneumococcal conjugate vaccine (PCV13) has been introduced targeting the same population. Overall (PPV23/ PCV13) vaccination coverage was 10\% (PCV13:6\%-August 2013). In January 2013, we implemented a two-year CAP surveillance among adults targeted by the vaccination program to monitoring the S.pneumoniae serotypes circulating in the region. We estimated preliminarily PCV13 effectiveness.

Methods: We conducted a test-negative design study using the information collected on patients with signs and/ or symptoms of CAP enrolled in the surveillance system. Cases were pneumonia patients with a specimen (sputum, blood) PCR positive for a PCV13-contained serotype. Controls were non-PCV13-serotypes pneumonia patients. The exposure of interest was PCV13 vaccination (regardless of previous PPV23 vaccination). We computed the test-negative estimate of vaccine effectiveness (VE) as 1-OR and calculated its exact $95 \%$ confidence interval. We analysed data using Stata/MP 11.2.

Results: We included 22 cases and 3 controls. Most cases and controls had at least one co-morbidity (i.e. chronic pulmonary or cardiovascular disease); two and one respectively were vaccinated for PCV13. Crude Vaccine Effectiveness was $80 \%(-1660 \% ; 99.3 \%)$.

Conclusion: The test-negative design provides relatively accurate VE estimates. However, due to the small number of patients, our findings, although suggestive of good vaccine effectiveness, should be interpreted with extreme caution. Significant efforts should be oriented to increase PCV13 vaccine uptake.

Conflict of interest 
ISPPD-0130

Controlling Pneumococcal Disease Around the Globe

\title{
DECLINING INCIDENCE AND CHANGING SEROTYPES IN PEDIATRIC INVASIVE INFECTIONS ACCOMPANY THE INTRODUCTION OF EXPANDED VALENCY PNEUMOCOCCAL CONJUGATE VACCINES IN PORTUGAL
}

\author{
S.I. Aguiar ${ }^{1}$, M.J. Brito ${ }^{2}$, A.N. Horácio ${ }^{1}$, J.P. Lopes ${ }^{1}$, M.J. Ramirez ${ }^{1}$, J.P. Melo-Cristino ${ }^{1}$ \\ ${ }^{1}$ Instituto de Microbiologia, Instituto de Medicina Molecular, Lisbon, Portugal; ${ }^{2}$ Pediatrics, Centro Hospitalar de Lisboa Central, Lisbon, Portugal
}

Background and Aims: The extended valency pneumococcal conjugate vaccines became available in Portugal in mid-2009 (PCV10) and early-2010 (PCV13). This study aimed at documenting the potential effects of children vaccination in the pneumococcal population associated with infection.

Methods: Between July 2008 and June 2012 a total of 471 cases of IPD recovered from patients $<18$ yrs were reported. Among these 392 isolates were available for serotyping and antimicrobial resistance profiling.

Results: The overall incidence of invasive pneumococcal disease (IPD) in the pediatric population declined from 8.19 cases/100,000 children in 2008-2009 to 4.52 in 2011-2012. IPD due to the serotypes included in the 7-valent conjugate vaccine (PCV7) in infants and toddlers remained constant, in spite of over 10 years of vaccine use, although it declined in older children. The decrease in IPD resulted from: 1 ) declines in the additional serotypes included in PCV10 and PCV13 (1,5, 7F), particularly serotype 1, in older children and, 2) to massive reductions of the additional serotypes included in PCV13 (3, 6A, 19A), particularly serotype 19A, in younger children. The delayed effect in serotype 19A and the simultaneous decline of serotype 1 in all groups suggests an important role of PCV13 in the former but that other factors may have triggered the decline of the latter possibly intensified by vaccination. The small rise in IPD caused by non-vaccine serotypes only marginally compensated for the decline in PCV13 serotypes.

Conclusion: PCV13 serotypes remain major causes of IPD, highlighting the potential role of enhanced vaccination in reducing pediatric IPD in Portugal.

No conflict of interest

ISPPD-0512

Controlling Pneumococcal Disease Around the Globe

\section{CHANGES IN CLINICAL FEATURES OF IPD IN CHILDREN, BEFORE AND AFTER 7-VALENT PNEUMOCOCCAL CONJUGATE VACCINE (PCV7) PROGRAM IN CALGARY, CANADA}

\author{
L.J. Ricketson ${ }^{1}$, N.G. Conradi², O.G. Vanderkooi ${ }^{3}$, J.D. Kellner ${ }^{3}$ \\ ${ }^{1}$ Pediatrics, Alberta Children's Hospital, Calgary, Canada; ${ }^{2}$ Primary Care and Public Health Sciences, King's College, London, United Kingdom \\ ${ }^{3}$ Pediatrics, University of Calgary, Calgary, Canada
}

Background and Aims: Since the introduction of PCV7 in 2002 and PCV13 in 2010, the incidence of invasive pneumococcal disease (IPD) has decreased significantly in children while the predominant pneumococcal serotypes have changed. This study describes changes in the clinical features of IPD in children ( $<18$ years) before and after PCV7 and PCV13 introduction.

Methods: The Calgary Area Streptococcus pneumoniae Epidemiology Research (CASPER) study collects information on all IPD cases in Calgary. Descriptive comparisons and multinomial models compared IPD in the pre-vaccine (2000June 2002) and post-vaccine (July 2002-2011) periods, using ICU and inpatient admission as outcome measures. Results: The incidence of IPD cases in children decreased from an average of 14 cases/100,000/year in 2000/2001 to 4 cases/100,000/year in 2011. The median age of cases increased from 2.0 y to 3.3 y $(p=0.06)$. The proportion with a risk factor (apart from age) that is an indication for pneumococcal vaccine did not change. The proportion of cases hospitalized increased from $59 \%$ to $83 \%(P<0.001)$. The proportion admitted to ICU increased from $14 \%$ to $25 \%(p=0.06)$. Focal disease (meningitis, peumonia, empyema) compared to bacteremia without focus increased from $43 \%$ to $66 \%(p<0.001)$. Having IPD in the post-vaccine period increased odds of hospitalization (OR: $2.9 ; 95 \%$ $\mathrm{Cl}: 1.6-5.8)$. In the post-vaccine period, serotypes in PCV13 but not PCV7 caused most cases (42\%).

Conclusion: Clinical features of IPD have changed since PCV7 was introduced, with a shift towards more focal infections requiring hospitalization. Thus, although overall IPD cases have declined, disease that does occur is more severe.

Conflict of interest 
ISPPD-0509

Controlling Pneumococcal Disease Around the Globe

\section{EPIDEMIOLOGY OF INVASIVE PNEUMOCOCCAL DISEASE (IPD) IN CALGARY, ALBERTA BEFORE AND AFTER PCV7 AND PCV13 VACCINE INTRODUCTION}

L.J. Ricketson ${ }^{1}$, O.G. Vanderkooi ${ }^{1}$, J.C. MacDonald ${ }^{2}$, J.D. Kellner ${ }^{1}$

${ }^{1}$ Pediatrics, University of Calgary, Calgary, Canada; ${ }^{2}$ Population and Public Health, Alberta Health Services, Calgary, Canada

Background: After the introduction of the 7-valent pneumococcal conjugate vaccine (PCV7) in 2002, IPD caused by PCV7 serotypes has been nearly eliminated at all ages. The purpose of this analysis is to describe recent trends in IPD (post-PVC7) in all age groups, as well as early trends following the introduction of the PCV13 in July 2010. Methods: We looked at overall and age-specific incidence of IPD caused by PCV7, PCV13 (less PCV7) and other serotypes from 1998-2012 in all age groups in Calgary. We also looked at changes in the proportion of IPD caused by specific serotypes and serotype groups.

Results: IPD has declined in the youngest and oldest age groups since PCV7 was introduced. The average incidence of 11 cases/100,000 people/year in 1998-2001 declined to 8 cases/100,000 people in 2012. Serotype distributions have also changed.
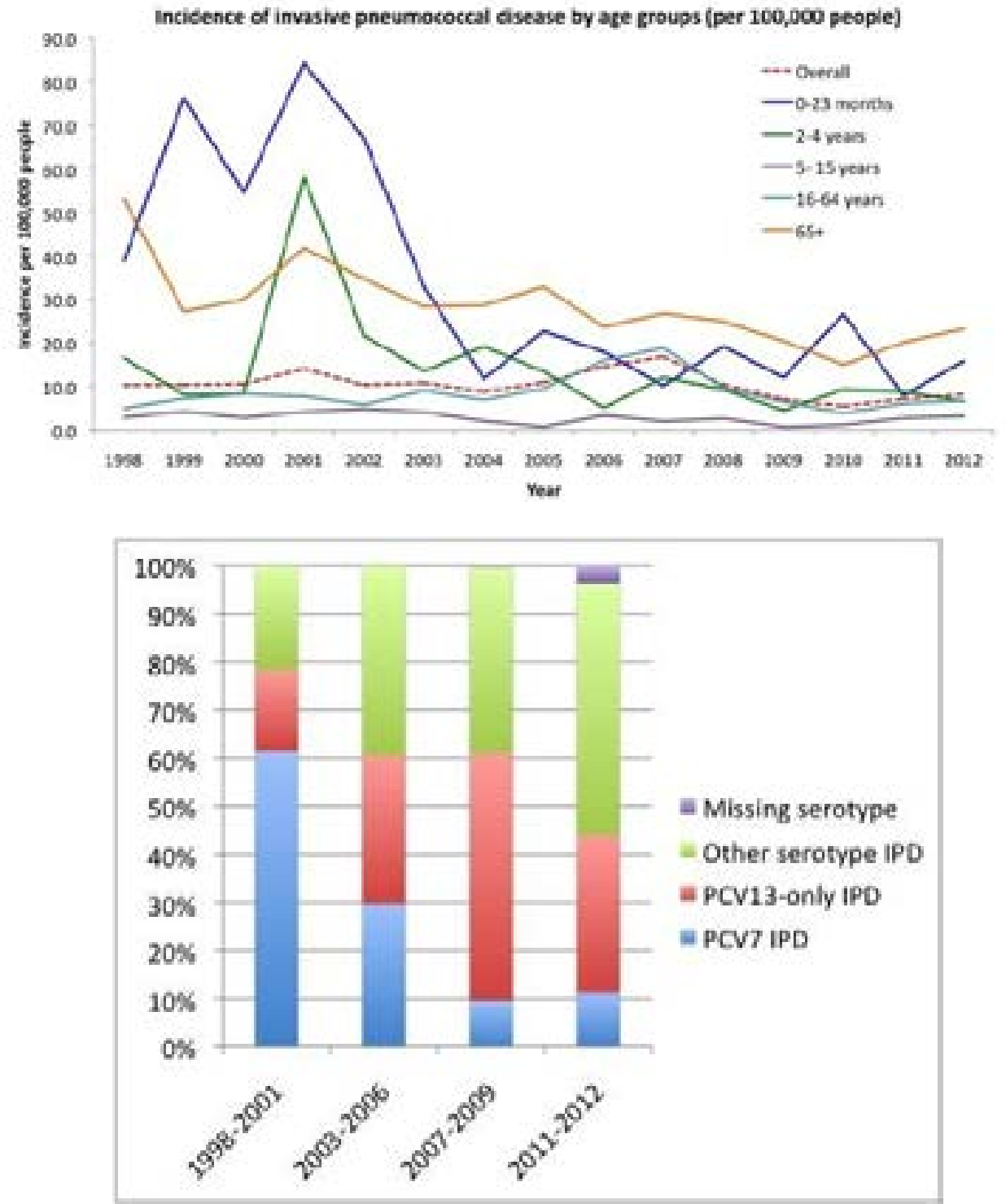

Conclusion: In the most vulnerable age groups, the very young and very old, there has been a decline in overall IPD since the introduction of conjugate vaccines. Overall incidence in the Calgary area has declined since 2007. PCV13 serotypes still cause IPD, especially in the older age groups.

Conflict of interest 
ISPPD-0325

Controlling Pneumococcal Disease Around the Globe

\title{
PNEUMOCOCAL SEROTYPES FROM ADULTS WITH INVASIVE DISEASE AND POTENTIAL VACCINE COVERAGE. SIREVA PROGRAM, VENEZUELA 1999-2012
}

\author{
A. Risquez ${ }^{1}$, E. Spadola ${ }^{2}$, L. Echezuría ${ }^{3}$, M.E. Landaeta ${ }^{4}$, F. de Abreu ${ }^{5}$ \\ ${ }^{1}$ Facultad de Medicina Escuela Luis Razetti Cátedra de Salud Pública, Universidad Central de Venezuela, Caracas, Venezuela; ${ }^{2}$ Bacteriología, In- \\ stituto Nacional de Higiens, Caracas, Venezuela; ${ }^{3}$ Medicina Preventiva y Social, Universidad Central de Venezuela Facultad de Medicina, Caracas, \\ Venezuela; ${ }^{4}$ Infectología, Hospital Universitario de Caracas Facultad de Mediicna UCV, Caracas, Venezuela; ${ }^{5}$ Infectología, Centro Médico de Cara- \\ cas, Caracas, Venezuela
}

Background and Aims: Pneumococcal serotypes that affect adults are changing throughout time. The objective is to describe pneumococcal serotypes from patients with invasive disease and associate them with theoretical vaccine coverage.

Methods: Epidemiological study of pneumococcal serotypes obtained from adults by the National Surveillance System (SIREVA), 1999 to 2012. Data obtained from the National Institute of Hygiene. Descriptive epidemiology of gender, age (adolescents 15-19; Young adults 20-49, adults 50-64, and elderly 65 and more years), type of sample, serotypes, an theoretical vaccine coverage. Estimation of potential vaccine coverage for VPP23v, VPC10v VPC13v. Definitions for maximum coverage includes any serotype incorporated in each vaccine; meanwhile, minimum coverage excludes non-specific identification of serotype among groups.

Results: 133 samples; 82 from males, with similar gender distribution among age groups. 68\% samples were from Cerebrospinal fluid, $13,5 \%$ pleural fluid, $13.5 \%$ blood and others, Most frequent serotypes were: 3 (14:10,5\%), 7F $(11 ; 8.3 \%)$, and 19A, 19F, 23F, Group 12 (each 8;6\%). Other serotypes found were: 14, 9V, 18C, 4, 5, 6B, POOL H (between 5.5 and $3 \%$ each), and the rest, less than $3 \%$ each, only 7 were not typed. During the last 4 years the number of specimens has increased (46.6\%). Serotype changes within age groups were: Serotypes 3 and 19A in first positions among all age groups. Vaccine coverage are PPV23v 81-71\%, and PCV13v 69-67\%

Conclusion: Pneumococcal serotypes from invasive samples differ more among adult groups of age. Surveillance for adults is increasing. Vaccines provide good coverage of invasive pneumococcal serotypes in Venezuela.

Conflict of interest

ISPPD-0049

Controlling Pneumococcal Disease Around the Globe

\section{COMPONENTS OF A COMPREHENSIVE IMPACT EVALUATION OF THE 10-VALENT PNEUMOCOCCAL CONJUGATE VACCINE ON CARRIAGE AND DISEASE IN FIJI}

F.M. Russell ${ }^{1}$, E. Rafai ${ }^{2}$, C. Satzke ${ }^{3}$, M. Kama ${ }^{2}$, E.M. Dunne ${ }^{3}$, T. Ratu 4 , K. Bright ${ }^{5}$, M. Cama ${ }^{2}$, B.D. Porter ${ }^{3}$, J. Edmunds ${ }^{6}$, E. Tuivaga ${ }^{4}$, L. Tikoduadua ${ }^{4}$, R. Devi ${ }^{7}$, J. Kado $^{4}$, K. Mulholland ${ }^{3}$

${ }^{1}$ Paediatrics, The University of Melbourne, Parkville, Australia; ${ }^{2}$ Centre for Communicable Diseases, Ministry of Health, Suva, Fiji; ${ }^{3}$ Pneumococcal Group, Murdoch Childrens Research Institute, Melbourne, Australia; ${ }^{4}$ Paediatrics, Ministry of Health, Suva, Fiji; ${ }^{5}$ International Health, Menzies School of Health Research, Darwin, Australia; ${ }^{6}$ Infectious Disease Epidemiology, London School of Hygiene and Tropical Medicine, London, United Kingdom; ${ }^{7}$ Family Health, Ministry of Health, Suva, Fiji

Background and Aims: In 2012, Fiji introduced the 10-valent pneumococcal conjugate vaccine (PCV10) using a $3+0$ schedule with AusAID support. After 3 years, the Ministry of Health will absorb all vaccine costs. Our impact evaluation will measure the direct and indirect effects of PCV10 on carriage, invasive pneumococcal disease (IPD), and pneumonia. To further understand the ethnic differences in disease rates, we will model carriage transmission. Methods: Annual community carriage surveys are being undertaken pre- and post-PCV10 introduction to measure direct and indirect effects in young infants, vaccinated children, and their caregivers. The frequency and intensity of social contact will be measured and the effect this has on pneumococcal carriage between ethnic groups will be compared. IPD surveillance will evaluate the impact of PCV10 on IPD and monitor invasive serotypes in all ages. An interrupted time series analysis will be undertaken pre/post PCV10 using inpatient and outpatient pneumonia data in all ages, identified by ICD10 codes. Radiological pneumonia and very severe pneumonia rates will be compared pre/post PCV10 in children less than two years old.

Conclusion: IPD surveillance data and special studies are required to fully measure the effect of PCV10 on IPD and pneumonia. This impact evaluation will provide the necessary data to justify the ongoing cost of the PCV10 program, ensure sustainability, and shed light on the reasons for ethnic differences in disease rates. In addition, the results will guide other countries in the region on the value of PCV10 on common childhood diseases.

No conflict of interest 


\title{
PREPARING FOR A BEFORE AND AFTER IMPACT EVALUATION OF PCV10 ON PNEUMONIA HOSPITALISATION RATES IN ALL AGES IN FIJI: BASELINE EPIDEMIOLOGY
}

\author{
F.M. Russell ${ }^{1}$, E. Rafai ${ }^{2}$, J. Kado ${ }^{3}$, M. Kama², L. Tikoduadua ${ }^{3}$, R. Devi ${ }^{4}$, K. Mulholland ${ }^{5}$ \\ ${ }^{1}$ Paediatrics, The University of Melbourne, Melbourne, Australia; ${ }^{2}$ Centre for Communicable Diseases, Ministry of Health, Suva, Fiji; ${ }^{3}$ Paediat- \\ rics, Ministry of Health, Suva, Fiji; ${ }^{4}$ Family Health, Ministry of Health, Suva, Fiji; ${ }^{5}$ Pneumococcal Group, Murdoch Childrens Research Institute, \\ Melbourne, Australia
}

Background and Aims: Despite pneumonia being the commonest cause of childhood admissions and deaths globally, there are very little data on the impact of PCV on pneumonia, and even less data on its indirect effects, particularly in resource-limited settings. In 2012, the Fiji Ministry of Health introduced PCV10. To prepare for an evaluation of the direct and indirect effects of PCV10, this study describes the epidemiology of hospitalised pneumonia in all ages, prior to PCV10 introduction.

Methods: All-cause pneumonia admissions for all ages were extracted using ICD10 codes from 2007-2011, from the Fiji national computerised hospital database and the manual reporting system, for those hospitals that are not computerised. The population denominators were sourced from the 2007 Census.

Results: There were 14,826 admissions of all-cause pneumonia. The age distribution followed a U-shaped curve with the highest annual incidence occurring in $<5$ year olds $(2,404$ per 100,000$)$ followed by the $65+$ year old age group (1,004 per 100,000). The incidence rate was up to 3.1 fold higher in the $\mathrm{i}$-Taukei (Indigenous) population compared with Fijians of Indian ethnicity. Of those with a fatal outcome, 30.2\% $(n=292)$ occurred in children aged $<5$ years (CFR $3.1 \%$ ) and $34 \%(n=329)$ of all deaths occurred in $65+$ year olds (CFR $21.5 \%$ ).

Conclusion: Pneumonia admissions and deaths were more common in young children and the elderly. For unknown reasons, pneumonia is more common in the Indigenous population. This data provides a good baseline to evaluate the direct and indirect effects of PCV10 on hospitalised pneumonia.

No conflict of interest

ISPPD-0522

Controlling Pneumococcal Disease Around the Globe

\section{HOSPITAL-BASED SURVEILLANCE TO EVALUATE THE EARLY EFFECTS OF PNEUMOCOCCAL VACCINATION PROGRAM} IN SAO PAULO

\author{
M. Safadi ${ }^{1}$, E. Berezin ${ }^{1}$, F. Almeida ${ }^{1}$, M. Arnoni ${ }^{1}$, M.L. Guerra², M.C. Brandileone ${ }^{2}$ \\ ${ }^{1}$ Pediatrics, Faculdade de Ciências Médicas da Santa Casa de São Paulo, São Paulo, Brazil; ${ }^{2}$ Microbiology, Instituto Adolfo Lutz, São Paulo, Brazil
}

Background and Aims: The 10-valent pneumococcal conjugate vaccine (PCV10) was introduced into the Brazilian Immunization Program in a $3+1$ schedule for children $<2$ years of age in mid 2010. Coverage for the primary three doses schedule of the vaccine among infants reached $80 \%$ in late 2011 . The aim of this study was to evaluate the early effects of immunization on the incidence of invasive pneumococcal disease (IPD) and serotype distribution in São Paulo.

Methods: We performed a 9-year (2004 -2012) surveillance, at a large sentinel hospital in Sao Paulo, including all children $<2$ years admitted due to IPD. Isolates were sent to a reference laboratory (Adolfo Lutz Institute) for serotyping and testing for antimicrobial susceptibility

Results: We identified 94 cases of IPD in children aged $<2$ years during the study period. The annual number of PCV10-types IPD and all-types IPD in children aged $<2$ years declined $89 \%$ and $77 \%$, respectively, in the postvaccination period. Serotype 14 , responsible in the pre-vaccination period for $38 \%$ of the IPD cases, disappeared in the post-vaccination period. No increase in overall non-PCV10 type IPD was observed. Reduction in the rates of penicillin resistance was also demonstrated.

Conclusion: The introduction of PCV10 into the routine vaccination program provided a rapid and significant reduction in IPD cases among children aged $<2$ years, the age group targeted for vaccination. These encouraging results highlight the need of continued surveillance studies to assess the long term impact of this vaccination program.

Conflict of interest 
ISPPD-0253

Controlling Pneumococcal Disease Around the Globe

\section{IMPACT OF PCV10 VACCINATION ON PNEUMOCOCCAL CARRIAGE IN YOUNG CHILDREN IN FIJI}

C. Satzke ${ }^{1}$, E.M. Dunne ${ }^{1}$, E. Rafai ${ }^{2}$, B.D. Porter ${ }^{1}$, M. Kama², T. Ratu' ${ }^{2}$, M. Cama ${ }^{2}$, B.D. Ortika ${ }^{1}$, C.L. Pell ${ }^{1}$, M. Habib ${ }^{1}$, M.L. Nation ${ }^{1}$, J.A. Smyth ${ }^{1}$, K. Bright ${ }^{3}$, L. Tikoduadua' ${ }^{2}$ R. Devi ${ }^{2}$, J. Kado $^{2}$, E.K. Mulholland ${ }^{1}$, F.M. Russell ${ }^{1}$

${ }^{1}$ Pneumococcal Research, Murdoch Childrens Research Institute, Parkville, Australia; ${ }^{2}$ Ministry of Health, Suva, Fiji; ${ }^{3}$ Global and Tropical Health, Menzies School of Health Research, Darwin, Australia

Background and Aims: There is a paucity of vaccine impact studies in the Asia-Pacific region. Fiji introduced the 10-valent pneumococcal conjugate vaccine (PCV10) in 2012. Our 'New Vaccine Evaluation Project' will measure the impact of PCV10 on nasopharyngeal carriage, invasive pneumococcal disease, and pneumonia. Annual community carriage surveys are being undertaken pre- and post-PCV10 introduction to measure direct and indirect effects in children (5-8 wk, 12-23 mo, 2-<7 y) and their caregivers.

Methods: Nasopharyngeal swabs were collected from a representative sample of 500 healthy children aged 12-23 mth pre-PCV10 introduction. Swabs were collected, transported, stored and processed according to the new WHO guidelines. The dominant $\alpha$-haemolytic morphology, and an example of each morphological variant, were identified as pneumococci using standard methods and serotyped by latex agglutination/Quellung.

Results: Preliminary analysis of the pre-PCV10 swabs found 236 contained pneumococci, and 213 did not.

Additional testing is underway for 51 swabs. The pneumococcal-positive swabs contained 249 serotypes, of which 103 were vaccine-types; 13 swabs contained more than one serotype. Soon we will examine swabs taken one year post-PCV10 introduction, and present these results compared to the pre-PCV10 data.

Conclusion: Future analyses will examine the bacterial load in specimens and the genetic relatedness of the pneumococcal isolates. Ongoing surveys will assess the direct effects of PCV10 up to three years post-introduction and will be compared with carriage data from children with pneumonia; forming part of a comprehensive study to examine total vaccine impact, including direct and indirect effects on carriage and disease, in Fiji.

No conflict of interest

ISPPD-0238

Controlling Pneumococcal Disease Around the Globe

\section{DOES USE OF PROPHYLACTIC ANTIPYRETICS (PAP) AFFECT IMMUNE RESPONSE TO VACCINATION IN INFANTS?}

J. Wysocki ${ }^{1}$, K.J. Center ${ }^{2}$, J. Brzostek ${ }^{3}$, E. Majda-Stanislawska ${ }^{4}$, P. Giardina ${ }^{5}$, V. Sundaraiyer ${ }^{6}$, S. Patterson ${ }^{2}$, W.C. Gruber $^{5}$, D. Scott ${ }^{5}$, A. Gurtman ${ }^{5}$

${ }^{1}$ Department of Preventive Medicine, Poznan University of Medical Sciences, Poznan, Poland; ${ }^{2}$ Vaccine Research, Pfizer, Collegeville, USA;

${ }^{3}$ Zespol Opieki Zdrowotnej w Debicy, Zespol Opieki Zdrowotnej w Debicy, Debica, Poland; ${ }^{4}$ Department of Children's Infectious Diseases, Medical University of Lodz, Lodz, Poland; ${ }^{5}$ Vaccine Research, Pfizer, Pearl River, USA; ${ }^{6}$ inVentiV Health Clinical LLC, inVentiV Health Clinical LLC, Ann Arbor, USA

Background: Prophylactic antipyretics (PAP) are often used during routine vaccination of infants and toddlers to prevent fever. However, recent research suggests that PAP may reduce immune response to some pediatric vaccines. This study evaluated the effect of different PAP on immune response to the 13-valent pneumococcal conjugate vaccine (PCV13) and other routinely recommended vaccine antigens.

Methods: 908 healthy infants were enrolled and randomized to receive either paracetamol (P) or ibuprofen (I) in 1 of 4 PAP regimens, or to no PAP (control), after each vaccine dose:

\begin{tabular}{|l|c|l|l|}
\hline & \multicolumn{3}{l|}{ PAP* Administration } \\
\hline Group (G) & Vaccination 0 $\mathrm{h}$ & $6-8 \mathrm{~h}$ & $12-16 \mathrm{~h}$ \\
\hline 1 & - & $\mathrm{P}$ & $\mathrm{P}$ \\
\hline 2 & - & $\mathrm{I}$ & $\mathrm{I}$ \\
\hline 3 & $\mathrm{P}$ & $\mathrm{P}$ & $\mathrm{P}$ \\
\hline 4 & $\mathrm{I}$ & $\mathrm{I}$ & $\mathrm{I}$ \\
\hline 5 & - & - & - \\
\hline * PAP doses: $\mathrm{P}, 15 \mathrm{mg} / \mathrm{kg} /$ dose; $\mathrm{l}, 10 \mathrm{mg} / \mathrm{kg} /$ dose. \\
\hline
\end{tabular}

All subjects received PCV13 and DTaP/IPV/Hib/HBV at 2, 3, 4, and 12 months; blood was drawn at 5 and 13 months.

Results: 800 subjects (88.1\%) were included in the primary analysis. Pneumococcal anticapsular IgG geometric mean concentration (GMCs) were significantly $(p<0.0125)$ lower in $\mathrm{G} 3$ versus $\mathrm{G} 5$ (control) for 5 of 13 serotypes after the primary series. Pertussis FHA and tetanus IgG GMC were significantly lower among G4 versus G5 after the primary series. No differences were observed for any antigens after the toddler dose.

Conclusion: Prophylactic paracetamol may interfere with primary series immune response to pneumococcal antigens. Ibuprofen did not interfere with pneumococcal responses, but may reduce response to pertussis FHA and tetanus antigens. These effects were not observed following the toddler dose. The clinical significance of these findings is unclear but suggests that PAP should be given careful consideration in the setting of infant vaccination. 
ISPPD-0460

Controlling Pneumococcal Disease Around the Globe

\title{
POSTMARKETING SAFETY ASSESSMENT OF 13-VALENT PNEUMOCOCCAL CONJUGATE VACCINE (PCV13) IN ROUTINE USE
}

\author{
R. Baxter ${ }^{1}$, K.J. Center², L. Aukes ${ }^{1}$, A. Yee ${ }^{1}$, A. Gurtman², D. Scott², W.C. Gruber ${ }^{2}$ \\ ${ }^{1}$ Vaccine Research, Kaiser Permanente Vaccine Study Center, Oakland, USA; ${ }^{2}$ Vaccine Research, Pfizer Vaccine Clinical Research, Colle- \\ geville and Pearl River, USA
}

Background: PCV13 was approved in February 2010 and recommended for use starting at approximately 2 months of age. A meta-analysis of prelicensure safety data in 4700 children demonstrated comparability of the safety profiles of PCV13 and PCV7. This post-licensure study further characterizes the safety profile of routinely administered PCV13.

Methods: This is an observational safety study of infants starting PCV13 vaccination in the first 6 months of life. Medical utilization data, coded according to standard practices, were obtained from Hospital, Emergency Room (ER) and Clinic databases for the Northern California Kaiser Permanente (NCKP) health system between 06/01/2010 and $06 / 30 / 2013$ and linked with NCKP's immunization registry. The primary analysis was event rates in time windows immediately following vaccination, compared with rates occurring 31-60 days after vaccination (self-control window) by dose and for the primary series combined, in each healthcare setting. Comparisons evaluated relative risks and exact two-sided $90 \%$ confidence intervals (Cls).

Results: During the observation period, 53,902 children received 176,664 doses of PCV13. Data from the full population are currently being analyzed. Findings from an interim analysis of 43,935 children who completed the primary series demonstrate an increase in events that are either expected following vaccination (eg, fever) or that commonly occur in young infants, such as dermatitis, respiratory tract infections, etc. No new safety signals have been identified.

Conclusion: Interim analysis from this large-scale study supports the satisfactory safety profile and continued use of Prevnar 13 as a routine infant vaccine. Final analyses will be presented.

Conflict of interest

ISPPD-0456

Controlling Pneumococcal Disease Around the Globe

\section{CHANGES IN INCIDENCE AND SEROTYPE DISTRIBUTION IN INVASIVE PNEUMOCOCCAL DISEASE (IPD) AFTER INTRODUCTION OF PREVNAR 13 IN KAISER NORTHERN CALIFORNIA}

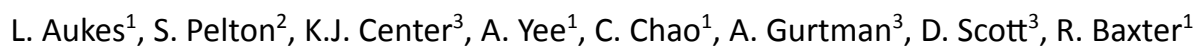

${ }^{1}$ Vaccine Research, Kaiser Permanente Vaccine Study Center, Oakland, USA; ${ }^{2}$ Vaccine Research, Boston University School of Medicine and Public Health, Boston, USA; ${ }^{3}$ Vaccine Research, Pfizer Vaccine Clinical Research, Collegeville, USA

Background: Since introduction of 7-valent pneumococcal conjugate vaccine (PCV7) for infants in the US in 2000, IPD has significantly decreased. The 13-valent vaccine (PCV13) was approved and recommended for children aged 2 through 59 months in the US in 2010 based on immunologic non-inferiority to PCV7. Ongoing surveillance for IPD following routine use of PCV13 is warranted to assess trends in disease including changes in serotype distribution. Methods: Active laboratory-based surveillance for IPD is ongoing since 2000 in Kaiser Permanente Northern California (population, 3.5 million). IPD is defined as isolation of Streptococcus pneumoniae from a usually sterile site. Rates of disease (per 100,000 members) were compared following introduction of and transition to PCV13 (May 2011 - April 2012) with a prior period, when PCV7 was in use (May 2008 - April 2010).

Results: Comparing PCV13 to PCV7 periods, IPD incidence rates (all serotypes) fell from 9.5 to 8.5 overall $(p=0.08)$ and from 12.0 to 5.3 in children $<6$ years of age $(p<0.01)$. A decrease in IPD caused by PCV13 serotypes from 4.7 to 3.7 overall $(p=0.02)$ and from 5.5 to 2.3 in children $<6$ years of age $(p=0.04)$ was observed. An increase in nonPCV13 serotype IPD from 3.3 to 4.2 overall $(p=0.03)$ and from 1.7 to 3.0 in children $<6$ years of age $(p=0.25)$ was also noted.

Conclusion: Preliminary results indicate a decline in overall IPD incidence as well as a change in serotype distribution in the post PCV13 time frame compared to the PCV7 era.

Conflict of interest 
ISPPD-0296

Controlling Pneumococcal Disease Around the Globe

\title{
IMPACT OF INTRODUCTION OF PNEUMOCOCCAL CONJUGATE VACCINE (PCV) INTO THE NATIONAL IMMUNIZATION PROGRAM ON THE RATE OF INVASIVE PNEUMOCOCCAL DISEASE (IPD) AND PENICILLIN NON- SUSCEPTIBLE STREPTOCOCCUS PNEUMONIAE (PRSP) IN SAUDI ARABIA
}

\author{
A. Shibl ${ }^{1}$, Z. memish ${ }^{2}$, A. senok $^{3}$
}

${ }^{1}$ Microbiology, King saud university, Riyadh, Saudi Arabia; ${ }^{2}$ infectious diseses, Ministry of health, Riyadh, Saudi Arabia; ${ }^{3}$ Microbiology, Alfaisal university, Riyadh, Saudi Arabia

Background and Aims: Between 2006 and 2012, a total of 1265 invasive pneumococcal disease (IPD) isolates from children under 5 years of age were collected from hospital laboratories around Saudi Arabia. These included 603 isolates before the introduction of PCV (2006 - 2008), 378 isolates during the introduction of PCV7 (2009 - 2010) and 284 isolates from the introduction of PCV13 (2011 - present).

Methods: All isolates were screened for serotyping using pneumococcal capsule-specific sera obtained from Statens Serum Institute (Copenhagen, Denmark).Penicillin susceptibility was determined using the E-test. The predominant IPD serotypes, before introduction of PCV7, were 23F, 19F, 4, 6B and 14. Following the introduction of PCV7, the predominant isolates belonging to serotypes were 1, 7F, 5 and 19A. After the introduction of PCV13, a reduction in serotypes 3, 19A, and 6A were observed.

Results and Conclusion: Compared to previous years, lower numbers of IPD isolates were obtained in 2013. In children under the age of five years, a significant reduction in the detection of penicillin non-susceptible Streptococcus pneumoniae (PRSP) isolates was observed from 49.7\% in 2009, 27.3\% in 2010, $19.2 \%$ in 2011 and $10.6 \%$ in 2012. Similarly in this age group, based on the number of invasive isolates received, the proportion of invasive pneumococcal disease due to vaccine serotypes, declined from $21 \%$ to about $4 \%$ in 2012 . These findings indicate that the introduction of the pneumococcal conjugate vaccine has had a positive impact in the country based on the reduction in the proportion of vaccine serotype IPD and a reduction in the frequency of PRSP.

No conflict of interest

ISPPD-0154

Controlling Pneumococcal Disease Around the Globe

\section{IMMUNOGENICITY AND SAFETY OF 13-VALENT PNEUMOCOCCAL CONJUGATE VACCINE IN JAPANESE ELDERLY ADULTS WHO ARE NAIVE TO PNEUMOCOCCAL VACCINE}

M. Shiramoto ${ }^{1}$, R. Hanada ${ }^{2}$, C. Juergens ${ }^{3}$, Y. Shoji ${ }^{4}$, M. Yoshida ${ }^{4}$, B. Ballan ${ }^{5}$, D. Cooper ${ }^{5}$, D. Scott ${ }^{5}$, W. Gruber ${ }^{5}$, B. Schmoele-Thoma ${ }^{3}$

${ }^{1}$ PS Clinic, Medical Co. LTA PS Clinic, Fukuoka, Japan; ${ }^{2}$ Clinical Research, Medical Co. LTA Sumida Hospital, Tokyo, Japan; ${ }^{3}$ Vaccine Clinical Research, Pfizer Pharma GmbH, Berlin, Germany; ${ }^{4}$ Vaccine Clinical Research, Pfizer Japan, Tokyo, Japan; ${ }^{5}$ Vaccine Clinical Research, Pfizer Vaccine Research, Pearl River, USA

Background and Aims: Clinical studies in adults from the United States and Europe have shown that the T-cell dependent 13-valent pneumococcal conjugate vaccine (PCV13) is safe and induces a greater functional immune response than the unconjugated T-cell independent 23-valent pneumococcal polysaccharide (PPSV23) vaccine for the majority of PCV13-serotypes. A similar comparison of PCV13 with PPSV23 was conducted for the first time in a Japanese study population $\geq 65$ years of age.

Methods: In a phase 3, randomized, modified double-blind, active-controlled trial, eligible subjects were randomized 1:1 to receive PCV13 or PPSV23 intramuscularly. Blood samples were collected immediately before and 1 month after vaccination for measurement of serotype specific anti-pneumococcal opsonophagocytic activity (OPA) titers.

Results: The evaluable analysis population included 366 PCV13 and 367 PPSV23 recipients. OPA geometric mean titers (GMTs) in the PCV13 group were statistically significantly higher than in the PPSV23 group for 9 of the 12 common serotypes and for serotype 6A, a serotype unique to PCV13; GMTs were similar for serotypes 1 and 14 and significantly lower for serotype 3 in the PCV13 group. Local reactions were more common in the PCV13 group but mainly mild to moderate; systemic and adverse events were similar across groups. There were no related serious adverse events.

Conclusion: As observed in global studies, PCV13 was safe and well tolerated and induced a greater functional immune response than PPSV23 for the majority of PCV13-serotypes in Japanese subjects. Japanese adults showed a similar pattern of responses as adults in the United States and Europe.

Conflict of interest 
ISPPD-0038

Controlling Pneumococcal Disease Around the Globe

\title{
INVASIVE PNEUMOCOCCAL DISEASE IN INFANTS YOUNGER THAN 90 DAYS BEFORE AND AFTER INTRODUCTION OF THE PNEUMOCOCCAL CONJUGATE VACCINE IN DENMARK
}

\author{
H. Slotved ${ }^{1}$, T.I.D. Dalby ${ }^{1}$, H.O.F. Hoffmann ${ }^{1}$ \\ ${ }^{1}$ Microbiology and Infection Control, Statens Serum Instítut, Copenhagen, Denmark
}

Background: The 7-valent pneumococcal conjugate vaccine (PCV7) was introduced into the Danish childhood immunization program (at 3, 5 and 12 months of age) in 2007, and was replaced with PCV13 in 2010. After the introduction of these vaccines the number of cases of invasive pneumococcal disease (IPD) due to vaccine types (VTs) declined markedly in children younger than two years of age. We present data on IPD in infants from a 14 year period.

Methods: The study included all infants (younger than 90 days) born 1st January 1999 until 30th June 2013, who had not been PCV vaccinated and from whom a pneumococcus isolate from blood or cerebrospinal fluid had been submitted to the national reference laboratory as part of the mandatory surveillance. All isolates were serotyped using pneumotest latex and Quellung reaction.

Results: A total of 58 IPD cases were identified (39 from 1999-2007, and 19 from 2008 and onwards). IPD cases due to PCV7 serotypes were not observed later than 2009. The incidence (number of IPD cases per 10,000 live births) varied from 0.45 to 0.93 in the period $1999-2007$ and from 0.17 to 0.92 in the period $2008-2013$.

Conclusion: In Danish infants IPD due to PCV7 serotypes has decreased, and has not been observed since 2009. The total number of IPD cases in Danish infants younger than 90 days seems to be unchanged.

Conflict of interest

ISPPD-0034

Controlling Pneumococcal Disease Around the Globe

\section{PREDICTION OF VACCINE- AND NON-VACCINE-TYPE INVASIVE PNEUMOCOCCAL DISEASE AMONG THE 50+ POPULATION AFTER CHANGES IN VACCINE POLICY IN NORWAY}

A. Steens ${ }^{1,2}$, D.F. Vestrheim ${ }^{1}$, B. Freiesleben De Blasio ${ }^{1}$

${ }^{1}$ Division of Infectious Disease Control, Norwegian Institute of Public Health, Oslo, Norway; ${ }^{2}$ European Programme for Intervention Epidemiology Training (EPIET), European Centre for Disease Prevention and Control (ECDC), Sweden

Background and Aims: Childhood immunisation with 7-valent pneumococcal conjugate vaccine (PCV7) since 2006 decreased vaccine-type (VT) invasive pneumococcal disease (IPD) and increased non-vaccine type (NVT)-IPD, also in non-targeted age groups. A 13-valent PCV (PCV13) replaced PCV7 in 2011. We aimed to predict how this changes VT and NVT IPD among the 50+ in order to inform vaccination strategies. Current recommendations for risk-groups including individuals aged 65 years and older are to use 23-valent polysaccharide vaccine (PPV23) which has 12 serotypes in common with PCV13. Uptake of PPV23 is thought to be 15-30\%.

Methods: We determined the monthly number of IPD caused by serotypes included in PCV13 (VT-PCV13), not included in PCV13 (non-PCV13) or included in PPV23 (VT-PPV23) in the 50+ between 1 January 2004 and 30 June 2013. We defined segmented quasi-Poisson models to characterize the recent trend (2004-June 2013) in monthly IPD counts, and used these models to predict expected seasonal counts for July 2013-June 2017.

Results: Preliminary results indicate a decrease in VT-PCV13 IPD from 191 cases in 2012-2013 to 76 (95\%PI 47-104) in 2016-2017. Non-PCV13 IPD will increase from 297 to 369 (95\%PI 326-412). This will lead to a net-decrease in VT-PPV23 IPD from 372 to 333 cases (95\%PI 289-377). Overall, IPD among the 50+ will decrease until 2015-2016 and stabilize subsequently at $445(95 \% \mathrm{PI} 391-499)$, if no change in vaccination policy occurs.

Conclusion: These results call for increased uptake of PPV23 among older adults and the need for pneumococcal vaccines conferring broader protections against IPD.

No conflict of interest

ISPPD-0099

Controlling Pneumococcal Disease Around the Globe

\section{SURVEILLANCE OF PNEUMOCOCCAL MENINGITIS AMONG CHILDREN IN UZBEKISTAN}

N.U. Tadjieva ${ }^{1}$, T.A. Daminov ${ }^{1}$, L.N. Tuychiev ${ }^{1}$, D.A. Tursunova ${ }^{1}$, D.F. Raimkulova ${ }^{1}$

${ }^{1}$ Infectious Diseases, Tashkent Medical Academy, Tashkent, Uzbekistan

Background: Information about the pneumococcal meningitis among children in Uzbekistan.

Methods: Surveillance of bacterial meningitis among children aged from 3 month to 14 years was set up at the Hospital of the Infectious diseases that has more than 40 pediatric admissions weekly, skilled personnel to perform lumbar punctures.

Results: A total of 210 children were admitted to the hospital with suspected acute bacterial meningitis, and pathogens were detected by culture or latex agglutination testing or BinaxNOW-Streptococcus Test or PCR-assay in $108(51.4 \%)$ of the purulent specimens. 108 confirmed cases were Streptococcus pneumoniae, and 2 (0.9\%) were Neisseria meningitidis. Approximately $55.6 \%$ of families reported de?nite prior antimicrobial use. The detected 
incidence rates of pneumococcal meningitis in Tashkent city in 2008 were 2.3 cases per 100,000 children aged $<14$ years, 2009 were 2.0, 2010 were 3.9 and in 2011 were 4.2 and 2012- 5.2 cases per 100,000 children aged <14 years. In 2012 the detected incidence rates of pneumococcal meningitis by age group were 2.6 cases per 100,000 children aged $<1$ year, 5.7 cases per 100,000 children aged $1-6$ year, 5.1 cases per 100,000 children aged $7-14$ years. Of the 108 patients with pneumococcal meningitis $24 \%$ had the adverse outcome and $1(0.9 \%)$ died during hospitalization.

Conclusion: The surveillance system detected a substantial burden of pneumococcal meningitis among children in Tashkent. S. pneumoniae was detected among $51.4 \%$ of investigated patients. The use of vaccines against this pathogen could prevent a substantial portion of disease and deaths in Uzbekistan.

No conflict of interest

\section{ISPPD-0128}

Controlling Pneumococcal Disease Around the Globe

\section{PNEUMOCOCCAL MENINGITIS: THE IMPACT OF THE PCV13 VACCINE AFTER 2-YEAR IMPLEMENTATION IN GREECE}

G. Tzanakaki ${ }^{1}$, A. Xirogianni ${ }^{1}$, K. Kesanopoulos ${ }^{1}$, M. Tsolia ${ }^{2}$, M. Theodoridou ${ }^{3}$, E. Roilides ${ }^{4}$, G. Syrogiannopoulos ${ }^{5}$, T. Georgakopoulou 6 , V. Spoulou', A. Argyropoulou ${ }^{8}$, A. Voyiatzi ${ }^{9}$, F. Markou ${ }^{10}$, J. Kremastinou ${ }^{1}$

${ }^{1}$ National Meningitis Reference Laboratory, National School of Public Health NSPH, Athens, Greece; ${ }^{2}$ Second Dept of Pediatrics P.\&A. Kyriakou Children's Hospital, University of Athens, Athens, Greece; ${ }^{3}$ First Dept of Pediatrics Aghia Sofia Children's Hospital, University of Athens, Athens, Greece; ${ }^{4}$ Third Dept of Pediatrics Hippokrateion Hospital, Aristotle University, Thessaloniki, Greece; ${ }^{5}$ Dept of Pediatrics, University of Thessaly, Larissa, Greece; ${ }^{6}$, Hellenic Center for Disease Control and Prevention, Athens, Greece; ${ }^{7}$ First Dept of Pediatrics Aghia Sofia Children's Hospital, University of Athens, Athens, Greece; ${ }^{8}$ Microbiology Laboratory, Evangelismos General Hospital, Athens, Greece; ${ }^{9}$ Microbiology Laboratory, Penteli Children's Hospital, Athens, Greece; ${ }^{10}$ Microbiology Laboratory, Serres General Hospital, Athens, Greece

Background: Streptococcus pneumoniae (SP) is a common cause of bacterial meningitis in Greece. PCV7 was introduced for children $<5$ years of age in 2006 and PCV13 in 2010. The aim of this study was to describe the epidemiology of pneumococcal meningitis (SPM) following the introduction of PCV13 in comparison with previous years.

Methods: Data of notified cases were reviewed retrospectively and were compared between three periods: $\boldsymbol{A}$ (prevaccine, 2000-2005), B (post PCV-7, 2006-2010) and C (post PCV13, 2011-2012). Three multiplex PCR (mPCR) assays were performed; one genus-specific and two for serotype identification.

Results: Of 598 SPM cases notified, 549 (91.8\%) were confirmed by culture $(n=22040 \%)$ and/or PCR $(n=418,76.2 \%)$. Overall annual incidence was estimated for the 2 periods (A\&B) at 0.54/100,000 while a reductionwas observed for period C (0.41/100 000). During period C, the overall incidence decreased in all age groups compared to period $B$ with a significant difference among the $0-4$ year olds (3.17 vs 2.2 vs $1.38 / 100,000$ for $\boldsymbol{A}, \boldsymbol{B}$ and $\boldsymbol{C}$ respectively, $\mathrm{p}<0.05)$. Most prevalent serotypes in period $\boldsymbol{A}$ were $\mathbf{1 4}(50 \%), \mathbf{2 3 F}(13.4 \%)$ and $\mathbf{6}(12.3 \%)$, while in $\boldsymbol{B} \mathbf{3}(31.3 \%), \mathbf{1 9 F}$ $(26.8 \%), 6(21.9 \%)$ and $19 A(12.5 \%)$. A considerable decrease in period $\boldsymbol{C}$ was observed for all serotypes especially 3 $(6.5 \%)$ and $19 \mathrm{~A}(2.6 \%)$ mainly found in older ages ( $>40$ years).

Conclusion: Incidence of SPM decreased significantly among younger children after the introduction of PCV13. The persistence of 3 and 19A in older ages is currently closely monitored.

No conflict of interest

ISPPD-0142

Controlling Pneumococcal Disease Around the Globe

\section{EFFECTS OF 5 YEARS OF IMMUNIZATION WITH HIGHER VALENT PNEUMOCOCCAL CONJUGATE VACCINES IN GERMAN CHILDREN}

M. van der Linden ${ }^{1}$, S. Perniciaro' ${ }^{1}$, M. Imöhl ${ }^{1}$

${ }^{1}$ National Reference Center for Streptococci Department of Medical Microbiology, University Hospital Aachen, Aachen, Germany

Background and Aims: A general recommendation for vaccination with pneumococcal conjugate vaccine (PCV) was issued for German children up to the age of 2 years in July 2006. In 2009, PCV10 and PCV13 were introduced in Germany. Here, we present data on cases of IPD from children collected in the eight years following the start of vaccination.

Methods: Pneumococcal isolates recovered from children with IPD were collected at the German National Reference Center for Streptococci (GNRCS). Identification of the isolates was confirmed and serotyping was performed using the Neufeld-Quellung reaction.

Results: In 2012-2013, IPD cases in children <2 years with PCV7 serotypes $(n=4)$ decreased by over $96 \%$ (compared to 1997-2006), while cases with non-PCV7 serotypes $(n=94)$ more than doubled. Particularly, serotypes $1,3,5$, 6A, 7F, 19A increased following PCV7 introduction, but decreased after higher-valent PCVs were introduced. In 2012-2013 only 20 cases with PCV13nonPCV7 serotypes were reported, as compared to 59 in 2009-2010 (-66\%). Reduction was observed for serotypes $1(-66 \%), 3(-50 \%), 6 \mathrm{~A}(-100 \%), 7 \mathrm{~F}(-82 \%)$ and 19A (-50\%). IPD cases due to Non-PCV13 serotypes increased strongly from 32 to $74(+124 \%)$, resulting in an increase in overall cases compared to $2011-2012$.

Conclusion: Eight years and five years following introduction of PCV7 and PCV13 for children in Germany, respectively, PCV7 serotypes have almost disappeared and PCV13 serotypes have strongly diminished among children $<2$ years. The strong increase in non-PCV13 serotypes might be related to a strong winter and influenza season. First data of 2013-2014 do not show a continuation of the high case numbers. 
ISPPD-0143

Controlling Pneumococcal Disease Around the Globe

\section{SEROTYPE DISTRIBUTION AND BURDEN OF PNEUMOCOCCAL DISEASE IN ADULTS IN GERMANY}

M. Imöhl ${ }^{1}$, S. Perniciaro ${ }^{1}$, M. van der Linden ${ }^{1}$

${ }^{1}$ National Reference Center for Streptococci Department of Medical Microbiology, University Hospital Aachen, Aachen, Germany

Objectives: In July 2006, PCV was recommended in Germany for children $\leq 24$ months. In this study, we present the serotype distribution among adults with IPD, with special regard to bacteremic pneumococcal pneumonia and IPD with underlying conditions before and after the start of childhood vaccination.

Methods: The German National Reference Center for Streptococci (GNRCS) has monitored the epidemiology of IPD in adults since 1992. Cases are reported by a laboratory-based surveillance system. These analyses include cases documented between 1992 and 2013. All isolates were serotyped using the Neufeld-Quellung reaction.

Results: In total, 18.315 isolates have been collected since 2008-2009 (average of 2100 isolates per season). Prior to the introduction of childhood vaccination (1992-2006), the most prevalent serotypes among adults with IPD were $14,3,4,9 \mathrm{~V}, 1,7 \mathrm{~F}$ and $23 \mathrm{~F}$, while in the period 2012-2013 serotypes 3, 19A, 7F, 22F and $12 \mathrm{~F}$ were most prevalent. The percentage of PCV7 serotypes in adults with IPD decreased from $40-45 \%$ before childhood vaccination to $7.5 \%$ in 2012-2013. In 2009, higher-valent vaccines (PCV10/PCV13) were introduced, resulting in a reduction of PCV13non-PCV7 IPD from $45.8 \%$ in $2009-2010$ to $36.1 \%$ in 2012-2013. In the season 2012-2013, the coverage of PCV13 in adults with IPD (all cases) was $43.7 \%$, in patients with bacteremic pneumococcal pneumonia $47.7 \%$ and in patients with underlying conditions $34.0 \%$.

Conclusion: Although a herd protection effect of PCV7 and PCV13 was observed, the burden of IPD, in particular of bacteremic pneumococcal pneumonia, among German adults is still high. Coverage of PCV13 is highest among patients with IPD and bacteremic pneumonia without underlying conditions.

Conflict of interest

ISPPD-0104

Controlling Pneumococcal Disease Around the Globe

\section{SEROTYPES IN ADULT INVASIVE PNEUMOCOCCAL DISEASE IN LATIN AMERICA BEFORE WIDESPREAD USE OF PCV13 AMONG PERSONS >50 YEARS OLD}

H. Vazquez ${ }^{1}$, V. Confalonieri ${ }^{2}$, M. Marín ${ }^{3}$, S. Fossati ${ }^{4}$, M. Regueira ${ }^{4}$, A. Corso ${ }^{5}$, D. Stamboulian ${ }^{1}$

${ }^{1}$ Vaccines for Adults, Fidec (Fighting Infectious Diseases in Emerging Countries), Miami FL, USA; ${ }^{2}$ Clinical Trial Division, Funcei (Fundación Centro de Estudios Infectológicos), Ciudad Autónoma de Buenos Aires, Argentina; ${ }^{3}$ Microbiology Service, Funcei (Fundación Centro de Estudios Infectológicos), Ciudad Autónoma de Buenos Aires, Argentina; ${ }^{4}$ Clinical Microbiology Service, National Institute of Infectious Diseases (INEI)-ANLIS “Dr. Carlos G. Malbrán”, Ciudad Autónoma de Buenos Aires, Argentina; ${ }^{5}$ Antimicrobial Service, National Institute of Infectious Diseases (INEI)-ANLIS “Dr. Carlos G. Malbrán”, Ciudad Autónoma de Buenos Aires, Argentina

Background: Since 2011, pneumococcal conjugate vaccine (PCV13) has been approved for use in adults $>50$ years of age in Latin American countries.

Methods: We assessed the distribution of Streptococcus pneumoniae serotypes isolated from invasive pneumococcal disease (IPD) in patients aged $50-59$ years and $>60$ years from Colombia, Chile, Argentina, Brazil, Uruguay and Mexico reported by Sireva II (2011-2012), and estimated serotypes covered by PCV13 and 23-valent pneumococcal polysaccharide vaccine (PPSV23). We included data from 43 IPD cases in Argentina (2011-2012), not yet published by SIREVA.

Results: From January 1st 2011 to December 31st 2012, 1713 IPD strains were reported (565, 50-59 years group; $1148,>60$ years group). Table 1 shows frequency distribution of most common serotypes by age group. No relevant differences were found between countries. In the 50-59 years age group, serotype coverage was 52.21\% (295/565) for PCV13 and 72.92\% (412/565) for PPSV23. In >60 year olds, PCV13 and PPSV23 covered 56.53\% (649/1148) and $75.35 \%(865 / 1148)$ of serotypes, respectively.

Table 1:

\begin{tabular}{|c|c|c|}
\hline & \multicolumn{2}{|c|}{ Age groups } \\
\hline & $50-59 y$ & $>60 y$ \\
\hline & $n=565$ & $n=1148$ \\
\hline \multirow[t]{6}{*}{ Serotypes (\%) } & 3 (9.02) & 3 (13.6) \\
\hline & $12 F(8.14)$ & 12F (6.97) \\
\hline & 7F (6.37) & $14(6.97)$ \\
\hline & $14(6.0)$ & 7F (5.49) \\
\hline & $19 F(4.95)$ & 19A (4.88) \\
\hline & $23 F(4.77)$ & $6 A(4.27)$ \\
\hline
\end{tabular}


Conclusion: In the 2-year period before wide use of PCV13 in adults, vaccine serotype coverage was, on average, $54 \%$ for PCV13 and 74\% for PPSV23. Most common serotypes were 3, 12F, 7F and 14. Continued surveillance of adult IPD in Latin America is warranted.

No conflict of interest

ISPPD-0153

Controlling Pneumococcal Disease Around the Globe

\title{
DISTRIBUTION OF PCV13 SEROTYPE STREPTOCOCCUS PNEUMONIAE IN ADULTS >/=50 YEARS WITH RADIOGRAPHICALLY-CONFIRMED COMMUNITY ACQUIRED PNEUMONIA (CAP); TWO HOSPITALS IN HONG KONG
}

\author{
Y. Yeung ${ }^{1}$, I. Fan Ngai Hung ${ }^{2}$, S. Gray ${ }^{3}$, E. Gonzalez ${ }^{3}$, M. Pride ${ }^{3}$, S. Shite ${ }^{4}$
}

${ }^{1}$ Department of Medicine and Geriatrics, Princess Margaret Hospital, Kowlooon, Hong Kong China; ${ }^{2}$ Department of Medicine, The University of Hong Kong Queen Mary Hospital, Hong Kong, Hong Kong China; ${ }^{3}$ Medicines Development Group, Pfizer Inc, Collegeville, USA; ${ }^{4}$ Medicines Development Group, Pfizer Inc, Pearl River, USA

Background and Aims: This prospective, multicenter study was conducted to evaluate the distribution of 13-valent pneumococcal conjugate vaccine (PCV13) serotypes in adults aged $\geq 50$ years with radiographically-confirmed community-acquired pneumonia (CAP) presenting to two hospitals in Hong Kong.

Methods: From December 2011 to May 2013, adults aged $\geq 50$ years presenting at two participating Hong Kong hospitals (Queen Mary Hospital and Princess Margaret Hospital) with clinically-suspected CAP (includes CAP or healthcare-associated pneumonia) and radiographic findings consistent with pneumonia were enrolled. Patients without radiographic confirmation of CAP were excluded from the final analysis. Demographic, healthcare exposure, and 23-valent pneumococcal polysaccharide vaccine (PPSV23) immunization data were collected. Urine was collected from all subjects; blood/respiratory samples were collected per standard of care. Streptococcus pneumoniae-positive CAP was defined by a positive result in $\geq 1$ of the following assays: 13-valent multiplex urinary antigen detection (UAD) assay developed by Pfizer to detect PCV13 serotypes (1, 3, 4, 5, 6A/C, 6B, 7F/A, 9V/A, 14, 18 $(\mathrm{C} / \mathrm{A}, \mathrm{B}, \mathrm{F}), 19 \mathrm{~A}, 19 \mathrm{~F}$, and 23F), BinaxNOW ${ }^{\circledR}$ S. pneumoniae, or microbiology cultures.

Results: 462 subjects were enrolled with radiographically-confirmed CAP. Of these 462 patients enrolled, 250 were enrolled at Queen Mary Hospital, and 209 were enrolled at Princess Margaret Hospital. Serotype distribution of S. pneumoniae-positive samples will be displayed across UAD, BinaxNOW ${ }^{\circledR}$, and blood/respiratory culture status. Further, these results will be presented by demographics, risk factors, significant medical history and vaccination status.

Conclusion: S. pneumoniae is an important etiology of CAP in adults aged $\geq 50$ years in Hong Kong.

Conflict of interest 